Supporting Information for:

\title{
An Electronic Rationale for Observed Initiation Rates in Ruthenium-mediated Olefin Metathesis: Charge Donation in Phosphine and $\mathbf{N}$-Heterocyclic Carbene Ligands
}

Kendra Getty, Mario Ulises Delgado-Jaime, and Pierre Kennepoh»*

The University of British Columbia, Department of Chemistry, Vancouver BC V6T 1 Z1

S1: Experimental Details \& Methodology

\section{S1.1 Synthesis}

All storage and manipulations of compounds were carried out under an inert atmosphere of nitrogen using standard Schlenk line or glove box techniques. The bispyridine complexes (3-6a and 3-6b) were synthesised similarly to published procedures $^{1-3}$ for $\mathbf{3} \mathbf{b}, \mathbf{4 a}$, and $\mathbf{4 b}$ by adding an excess of the appropriate pyridine to complexes $\mathbf{1 a}$ or $\mathbf{1 b}$. The pyridines are mono-substituted as follows: py $=3$-bromopyridine $(\mathbf{3} \mathbf{a}, \mathbf{b})$; pyridine $(\mathbf{4} \mathbf{a}, \mathbf{b}) ; 3$-nitropyridine $(\mathbf{5} \mathbf{a}, \mathbf{b})$; or 4 methoxypyridine $(\mathbf{6} \mathbf{a}, \mathbf{b})$. Toluene, ether and pentane were dried by passage through solvent purification columns. 3-bromopyridine was purchased from AlfaAesar and 4-methoxypyridine was purchased from Sigma-Aldrich; both were dried over $4 \AA$ molecular sieves. 4-nitropyridine was purchased from AlfaAesar and dried under vacuum. Complexes $\mathbf{1 a}$ and $\mathbf{1} \mathbf{b}$, and anhydrous pyridine were purchased from Sigma-Aldrich and used as received. Ampoules of methylene chloride- $\mathrm{d}_{2}$ were purchased from Cambridge Isotope Laboratories and used as received. Compounds $\mathbf{2} \mathbf{a}, \mathbf{2} \mathbf{b}, \mathbf{7 a}$, and $\mathbf{7 b}$ were synthesized as described elsewhere ${ }^{4,5}$ and obtained from Warren $\mathrm{E}$. Piers.

\section{S1.2 XAS Sample Preparation}

All sample preparation was performed in a nitrogen-filled glove box and samples were kept in an inert atmosphere or frozen in liquid nitrogen prior to and during data acquisition. Each solid was diluted approximately seven-fold with boron nitride and the mixture was gently ground for $>5$ minutes using a mortar and pestle to generate homogenous, finely dispersed powders. Each mixture was 
then pressed into a $0.5 \mathrm{~mm}$ thick Al spacer that was sealed on both sides with Kapton tape.

\section{S1.3 XAS Data Acquisition}

Ru K-edge X-ray absorption spectroscopic data were collected at the Stanford Synchrotron Radiation Laboratory (SSRL) on beamline 7-3 under ring conditions 80-100 mA at 3.0 GeV. This beamline has a 20-pole, 2 Tesla wiggler, $0.8 \mathrm{mrad}$ beam and a Si (220) double-crystal monochromator that was detuned by $50 \%$ intensity to attain harmonic rejection. The incident $\mathrm{x}$-ray intensity $\left(\mathrm{I}_{0}\right)$, sample absorption $\left(\mathrm{I}_{1}\right)$, and $\mathrm{Ru}$ reference absorption $\left(\mathrm{I}_{2}\right)$ were measured as transmittance using argon-filled ionization chambers. Two to four sweeps were taken for each sample and all data were measured to $k=18 \AA^{-1}$ at $13 \pm 3 \mathrm{~K}$ within an Oxford Instruments CF1208 continuous-flow liquid helium cryostat. See S2 for motor configurations used during data acquisition on SSRL BL7-3.

\section{S1.4 XAS Data Processing}

X-ray absorption data were processed using SIXPack, which was developed by Sam Webb at SSRL. ${ }^{6}$ All identical transmission sweeps for a complex were averaged and energy calibrated using Ru foil as an internal reference, with the lowest energy inflection point assigned as $22117 \mathrm{eV}$. Background subtraction and normalization were performed simultaneously using a linear pre-edge function and a quadratic post-edge function. PeakFit ${ }^{\circledR 7}$ was used to model the pre-edge region with a single Voigt amplitude function and the ionization edge feature with a cumulative Gaussian/Lorentzian function (See S3 \& S4 for details). 
S2: XAS Acquisition Details - Initial Motor Configuration on SSRL Beamline 7-3

\begin{tabular}{|c|c|}
\hline Motor & Position \\
\hline MOTOPSLT & 1.499 \\
\hline MOBOTSLT & 1.499 \\
\hline MOVERT & 5.311 \\
\hline MOPITCH & 0.012 \\
\hline MOBEND & 200.000 \\
\hline GIRDER & 0.000 \\
\hline MONOBOTSLT & 2.000 \\
\hline MONTOPSLT & 2.000 \\
\hline MONOBOTSLT & 22500.023 \\
\hline TABLEVERT & $88.733^{*}$ \\
\hline TABLEHORX & -9.279 \\
\hline TABLEPITCH & 0.000 \\
\hline S1VGAP & $1.000^{*}$ \\
\hline S1VTRAN & 0.000 \\
\hline S1HGAP & $5.000^{*}$ \\
\hline S1HTRAN & 0.000 \\
\hline CRYOHOR & $3.100^{*}$ \\
\hline CRYOVERT & $5.631^{*}$ \\
\hline
\end{tabular}

* denotes motors that were optimized for each run 
S3: Functions used in PeakFit ${ }^{\circledR}$ for pre-edge and ionization edge fitting

Pre-edge: Voigt Amplitude Function (PeakFit ${ }^{\circledR}$ )

$$
y=\frac{a_{0} \int_{-\infty}^{\infty} \frac{\exp \left(-t^{2}\right)}{a_{3}^{2}+\left(\frac{x-a_{1}}{a_{2}}-t\right)^{2}} d t}{\int_{-\infty}^{\infty} \frac{\exp \left(-t^{2}\right)}{a_{3}^{2}+t^{2}} d t}
$$

$$
\begin{aligned}
& \mathrm{a}_{0}=\text { amplitude } \\
& \mathrm{a}_{1}=\text { center } \\
& \mathrm{a}_{2}=\text { width }(>0) \\
& \mathrm{a}_{3}=\text { shape }(\geq 0)
\end{aligned}
$$

Ionization edge: Cumulative Gaussian/Lorentzian Function (see, for example, reference ${ }^{8}$ in supporting information)

$$
y=a_{4} \times \frac{a_{0}}{2}\left[1+\operatorname{erf}\left(\frac{x-a_{1}}{\sqrt{a_{2}}}\right)\right]+\left(1-a_{4}\right) \times \frac{a_{0}}{\pi}\left[\tan ^{-1}\left(\frac{x-a_{1}}{a_{3}}\right)+\frac{\pi}{2}\right]
$$

$$
\begin{aligned}
& a_{0}=\text { amplitude } \\
& a_{1}=\text { inflection point } \\
& a_{2}=\text { Gaussian width } \\
& a_{3}=\text { Lorentzian width } \\
& a_{4}=\text { shape }
\end{aligned}
$$


S4: Results from non-linear least-squares fitting of Ru K-edge data in the nearedge region using one pseudo-Voigt function (pre-edge) and a cumulative Gaussian/Lorentzian function (edge).

\begin{tabular}{|c|c|c|c|c|c|c|c|c|c|c|}
\hline \multirow[b]{2}{*}{ Complex } & \multirow{2}{*}{$\begin{array}{l}\text { Number } \\
\text { of } \\
\text { Sweeps } \\
\text { Averaged }\end{array}$} & \multicolumn{3}{|c|}{ Pre-edge } & \multicolumn{5}{|c|}{ Edge } & \multirow[b]{2}{*}{$r^{2}$} \\
\hline & & $\begin{array}{l}\text { Amp } \\
(\mathrm{a} 0)\end{array}$ & $\begin{array}{l}\text { Energy, } \\
\text { eV (a1) }\end{array}$ & $\begin{array}{c}\Delta \text { Energy, } \\
\mathrm{eV}\end{array}$ & $\begin{array}{l}\text { Amp } \\
(\mathrm{a} 0)\end{array}$ & $\begin{array}{l}\text { Energy, } \\
\text { eV (a1) }\end{array}$ & $\begin{array}{c}\Delta \text { Energy, } \\
\mathrm{eV}\end{array}$ & $\begin{array}{l}\text { Gaussian } \\
\text { Width (a2) }\end{array}$ & $\begin{array}{l}\text { Lorentzian } \\
\text { Width (a3) }\end{array}$ & \\
\hline $1 a$ & 4 & 0.21 & 22116.4 & \multirow{2}{*}{0.5} & 1.00 & 22122.7 & \multirow{2}{*}{0.8} & 3.9 & 5.1 & 1.0000 \\
\hline 1b & 3 & 0.21 & 22116.9 & & 0.99 & 22123.5 & & 5.3 & 4.3 & 0.9999 \\
\hline $2 a$ & 2 & 0.20 & 22117.1 & \multirow{2}{*}{0.5} & 0.99 & 22122.7 & \multirow{2}{*}{1.0} & $5.5^{*}$ & 5.1 & 0.9998 \\
\hline $2 b$ & 2 & 0.26 & 22117.5 & & 1.00 & 22123.7 & & $5.5^{*}$ & $5.5^{*}$ & 0.9997 \\
\hline $3 a$ & 2 & 0.11 & 22117.6 & \multirow{2}{*}{-0.1} & 0.99 & 22122.9 & \multirow{2}{*}{0.4} & 5.5 & 2.6 & 0.9999 \\
\hline $3 \mathbf{b}$ & 2 & 0.14 & 22117.5 & & 0.99 & 22123.3 & & 3.9 & 4.2 & 0.9999 \\
\hline $4 a$ & 2 & 0.11 & 22117.7 & \multirow{2}{*}{-0.2} & 0.99 & 22123.3 & \multirow{2}{*}{0.2} & 3.8 & 4.6 & 1.0000 \\
\hline 4b & 2 & 0.12 & 22117.5 & & 0.99 & 22123.5 & & 4.8 & 4.2 & 0.9999 \\
\hline $5 a$ & 2 & 0.12 & 22117.6 & \multirow{2}{*}{-0.2} & 1.00 & 22123.1 & \multirow{2}{*}{0.2} & 3.6 & 4.5 & 0.9999 \\
\hline $5 \mathbf{b}$ & 2 & 0.13 & 22117.4 & & 1.00 & 22123.3 & & 4.0 & 5.4 & 0.9999 \\
\hline $6 a$ & 3 & 0.11 & 22117.5 & \multirow{2}{*}{-0.1} & 0.99 & 22123.2 & \multirow{2}{*}{0.1} & 4.1 & 4.3 & 0.9999 \\
\hline 6b & 3 & 0.12 & 22117.4 & & 0.99 & 22123.3 & & 4.5 & 4.3 & 1.0000 \\
\hline $7 a$ & 2 & 0.30 & 22118.4 & \multirow{2}{*}{0.4} & 0.99 & 22123.4 & \multirow{2}{*}{0.8} & 3.3 & 4.5 & 0.9999 \\
\hline 7b & 2 & 0.33 & 22118.8 & & 0.99 & 22124.2 & & 3.5 & 5.5 & 0.9999 \\
\hline
\end{tabular}

* denotes fixed values

Additional Details: The spectra were fit over the energy region from $22090 \mathrm{eV}$ to $22128 \mathrm{eV}$. A pre-edge width (a2) of $3.1 \mathrm{eV}$ was used for all complexes as it successfully reproduced acceptable fits for the series of complexes and prevented distortions due to variations in the background. The pre-edge Gaussian ratio (a3) and edge Gaussian ratio (a4) were fixed at 0.5 for all complexes, i.e., 1:1 ratio of Gaussian and Lorentzian contributions. The Gaussian and Lorentzian edge widths averaged $4.4 \mathrm{eV}(1 \sigma=0.8 \mathrm{eV})$ and $4.6 \mathrm{eV}(1 \sigma=$ $0.8 \mathrm{eV})$, respectively. Compounds $\mathbf{2} \mathbf{a}, \mathbf{b}$ have broader edges than other compounds, so the edge widths were fixed to ensure reasonable values. The second derivative plots in Figure 1 were obtained from data that was smoothed using 5 point adjacent averaging. 
S5: Near-edge Ru K-edge XAS spectra for complexes $\mathbf{2 a}$ and $\mathbf{2 b}\left(\mathbf{a}: \mathrm{L}^{-} \mathrm{PC} y_{3}, \mathbf{b}\right.$ : $\mathrm{L}=\mathrm{H}_{2} \mathrm{IMes}$ ).

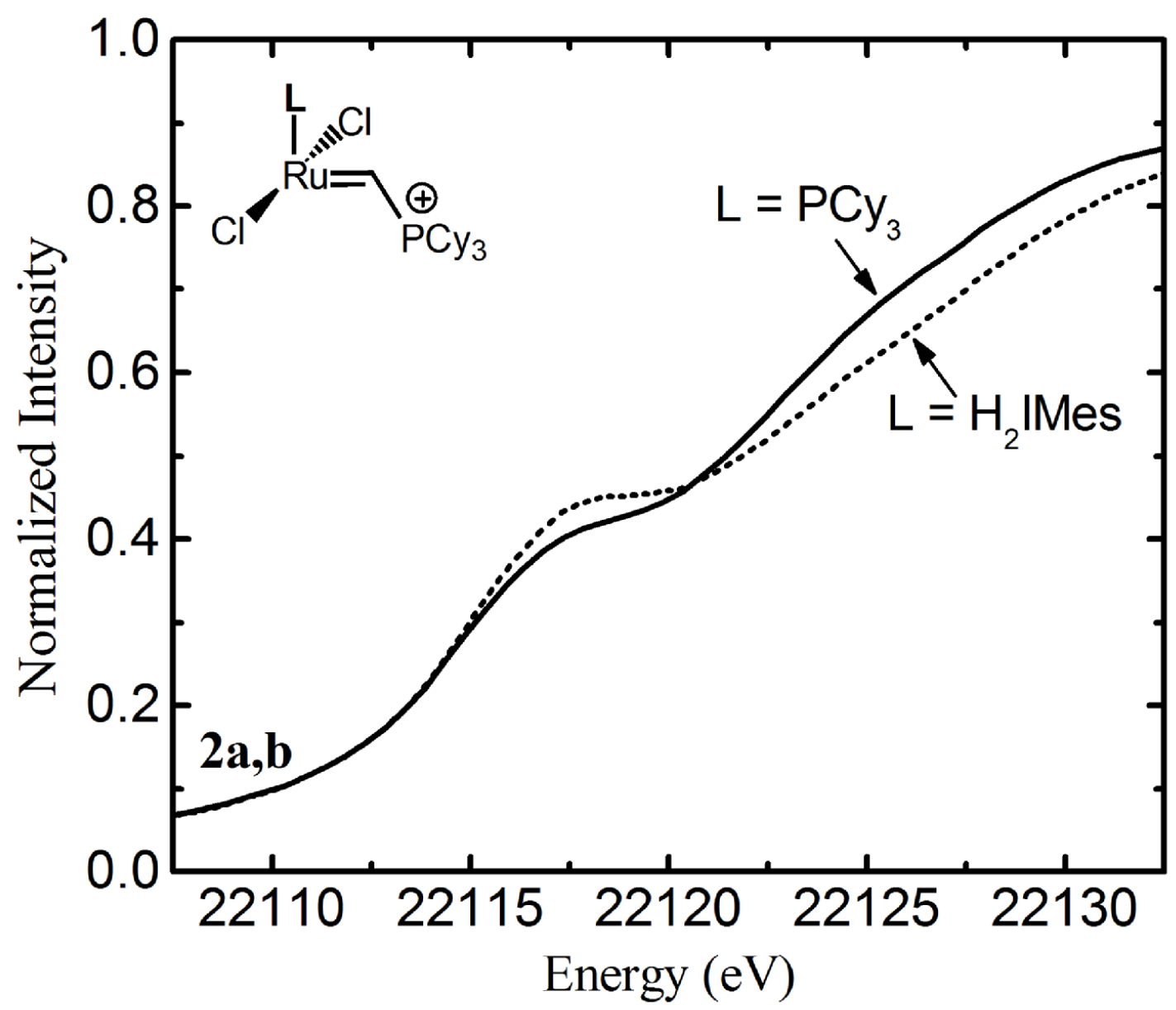


S6: Near-edge Ru K-edge XAS spectra for complexes $\mathbf{3 a}$ and $\mathbf{3 b}\left(\mathbf{a}: \mathrm{L}=\mathrm{PCy}_{3}, \mathbf{b}\right.$ : $\mathrm{L}=\mathrm{H}_{2} \mathrm{IMes}$ ).

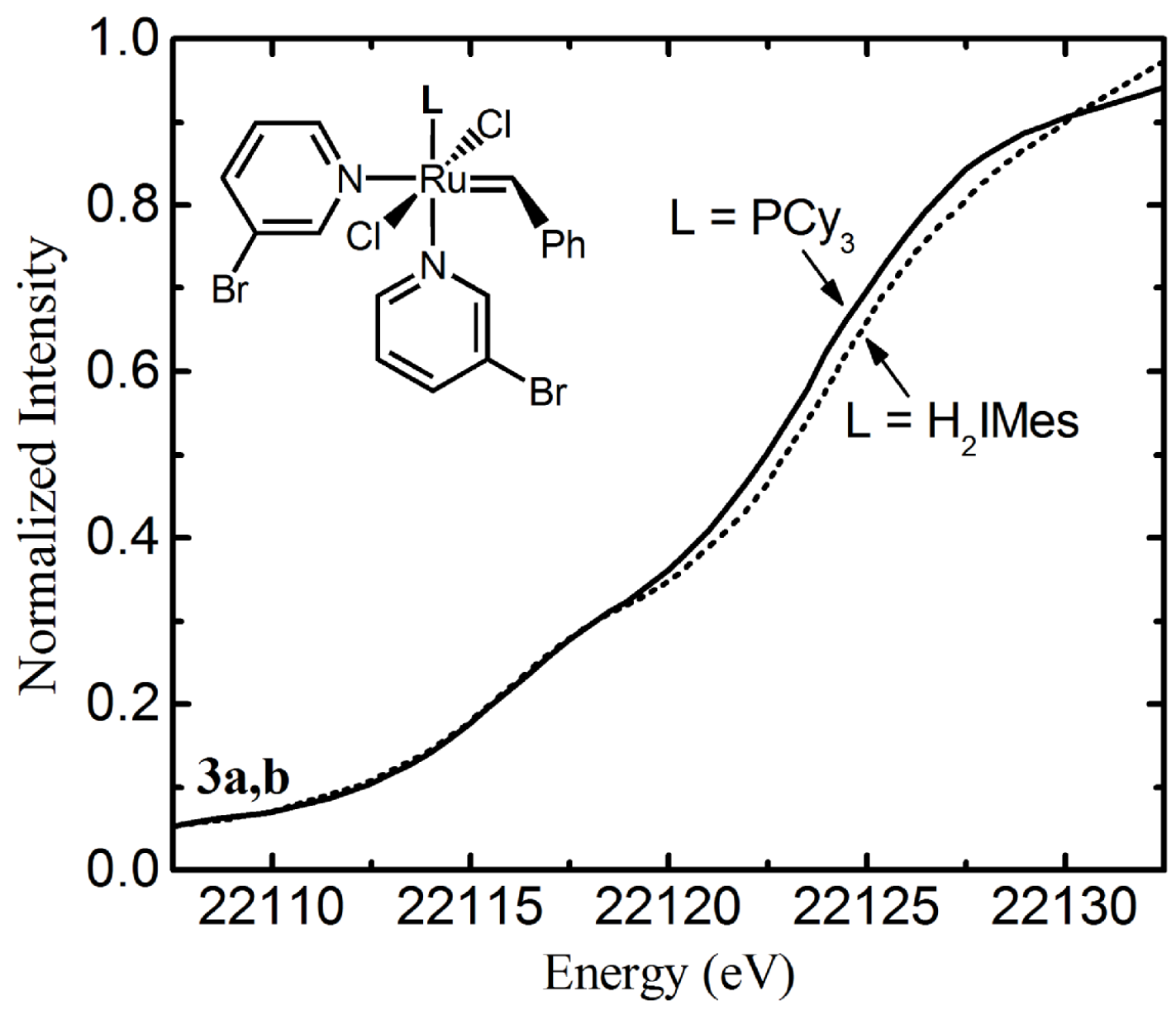


S7: Near-edge Ru K-edge XAS spectra for complexes $\mathbf{4 a}$ and $\mathbf{4 b}\left(\mathbf{a}: \mathrm{L}^{-} \mathrm{PC} y_{3}, \mathbf{b}\right.$ : $\mathrm{L}=\mathrm{H}_{2} \mathrm{IMes}$ ).

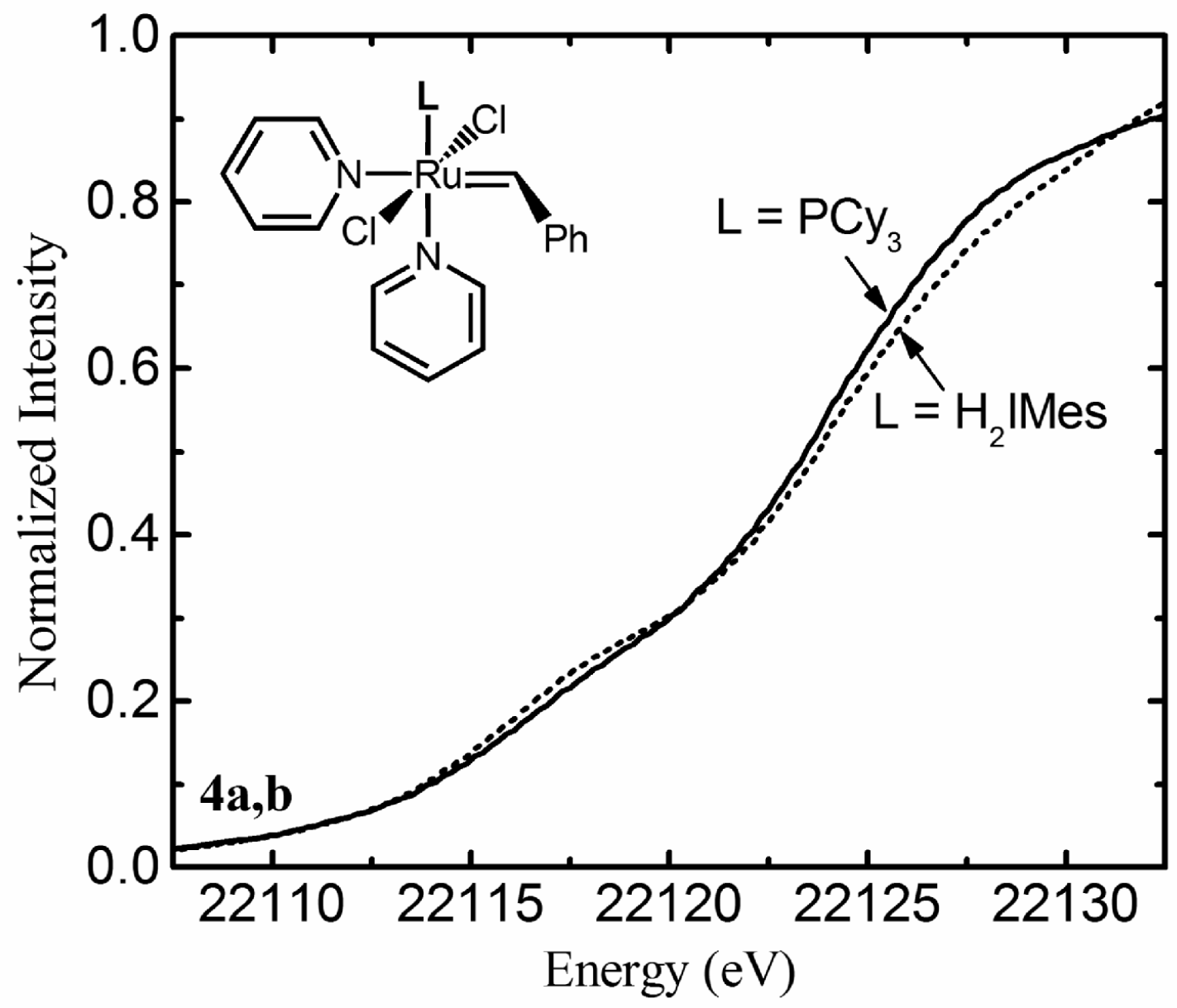


S8: Near-edge Ru K-edge XAS spectra for complexes $\mathbf{5 a}$ and $\mathbf{5 b}\left(\mathbf{a}: \mathrm{L}_{=} \mathrm{PC} y_{3}, \mathbf{b}\right.$ : $\mathrm{L}=\mathrm{H}_{2} \mathrm{IMes}$ ).

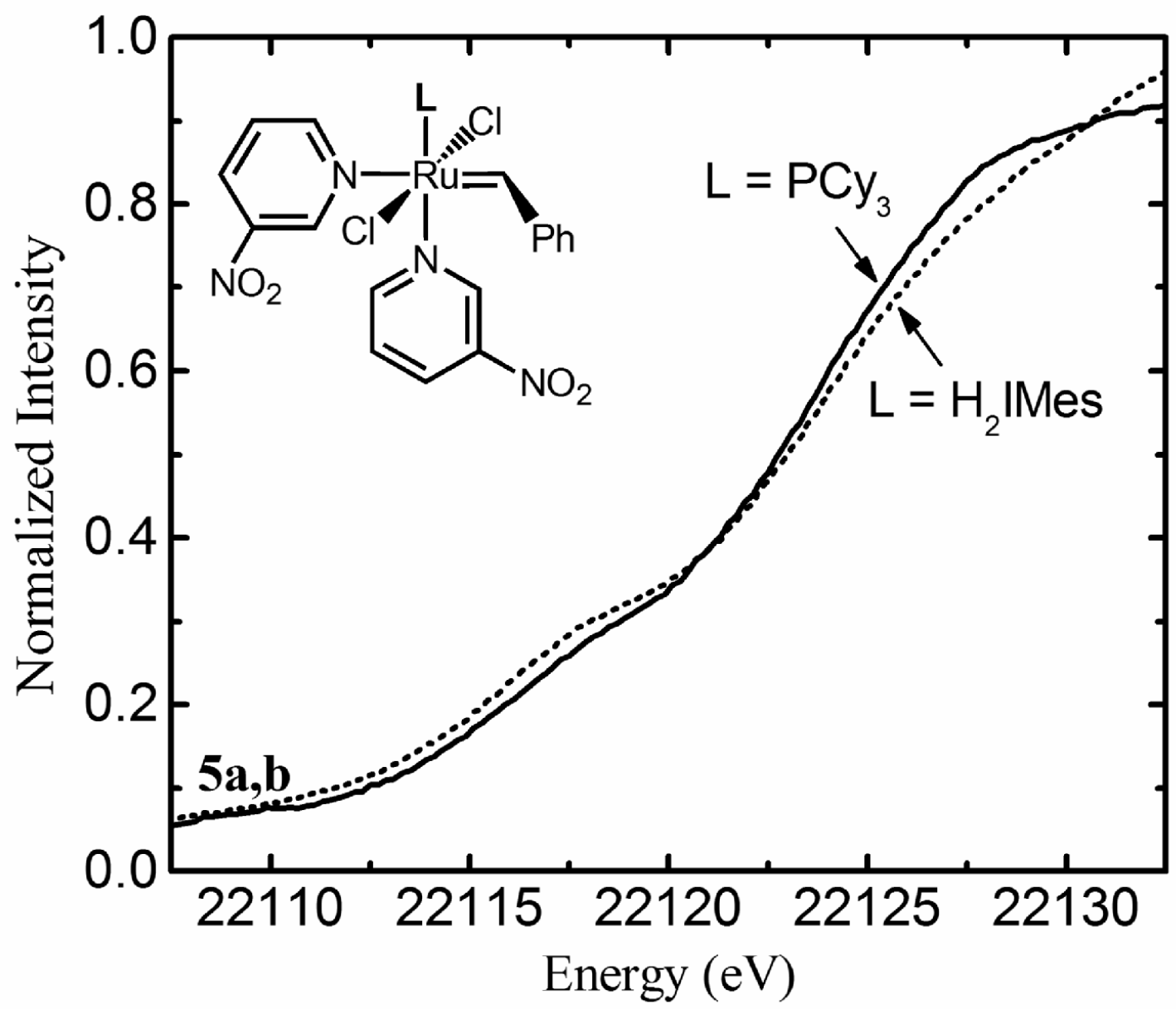


S9: Near-edge Ru K-edge XAS spectra for complexes $\mathbf{6} \mathbf{a}$ and $\mathbf{6 b}\left(\mathbf{a}: \mathrm{L}=\mathrm{PCY}_{3}, \mathbf{b}\right.$ : $\mathrm{L}=\mathrm{H}_{2} \mathrm{IMes}$ ).

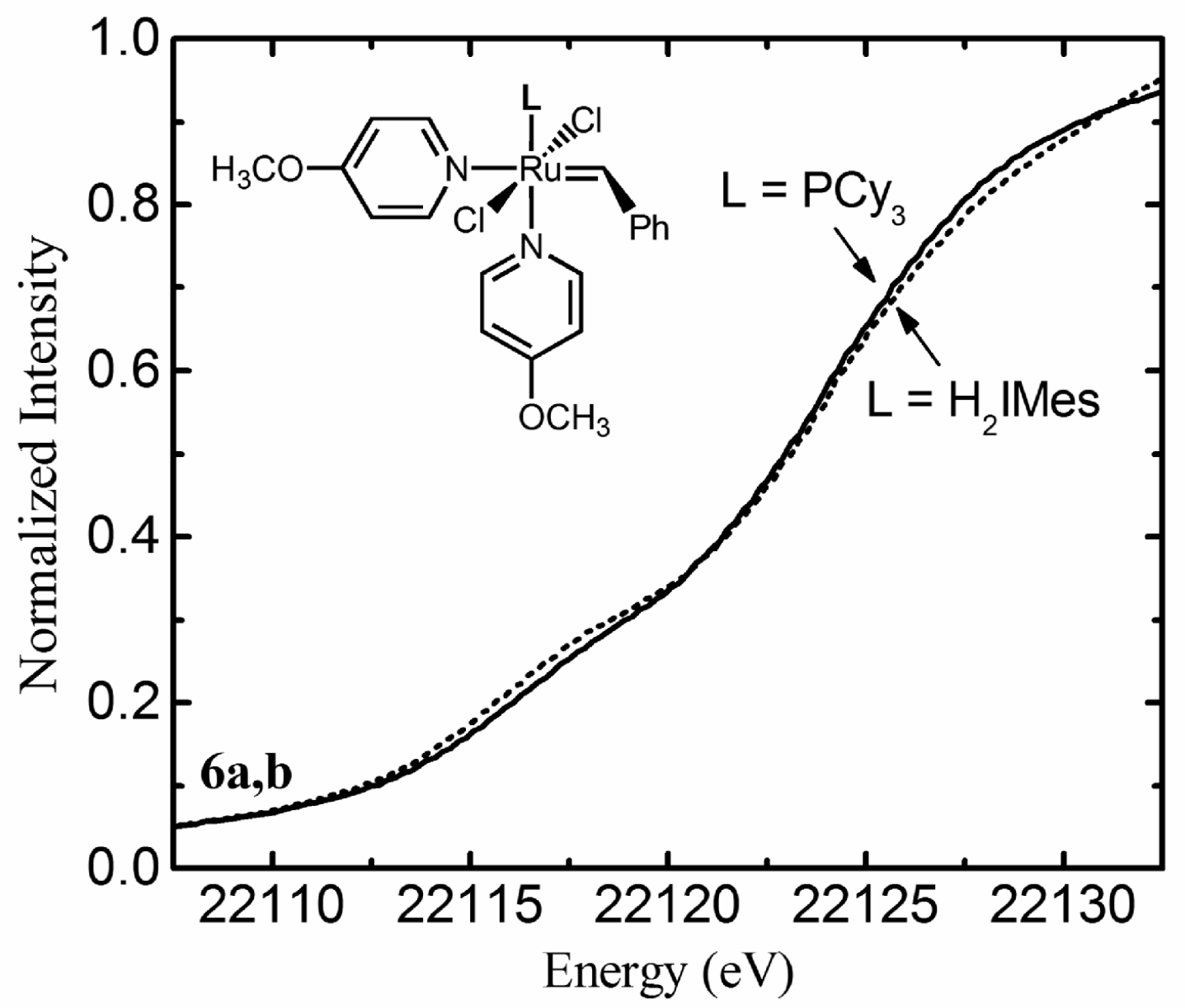


S10: Near-edge Ru K-edge XAS spectra for complexes 7a and 7b (a: $\mathrm{L}=\mathrm{PC} y_{3}, \mathbf{b}$ : $\mathrm{L}=\mathrm{H}_{2} \mathrm{IMes}$ ).

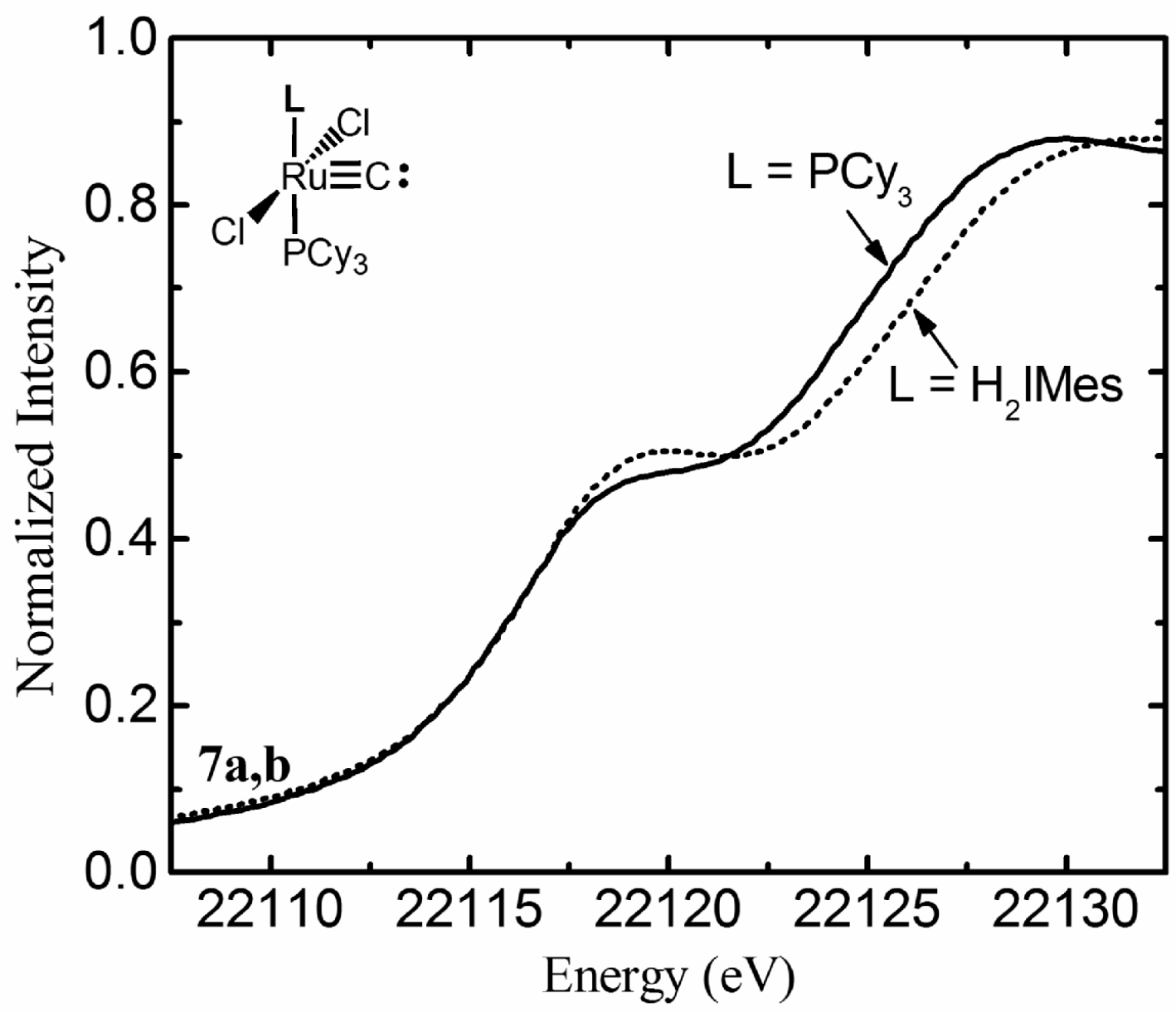


S11: Expanded Ru K-edge XAS spectra for complexes 1-7a and 1-7b (a: $\mathrm{L}=\mathrm{PC}_{3}, \mathbf{b}$ : $\left.\mathrm{L}=\mathrm{H}_{2} \mathrm{IMes}\right)$. The spectra have been vertically shifted for clarity.

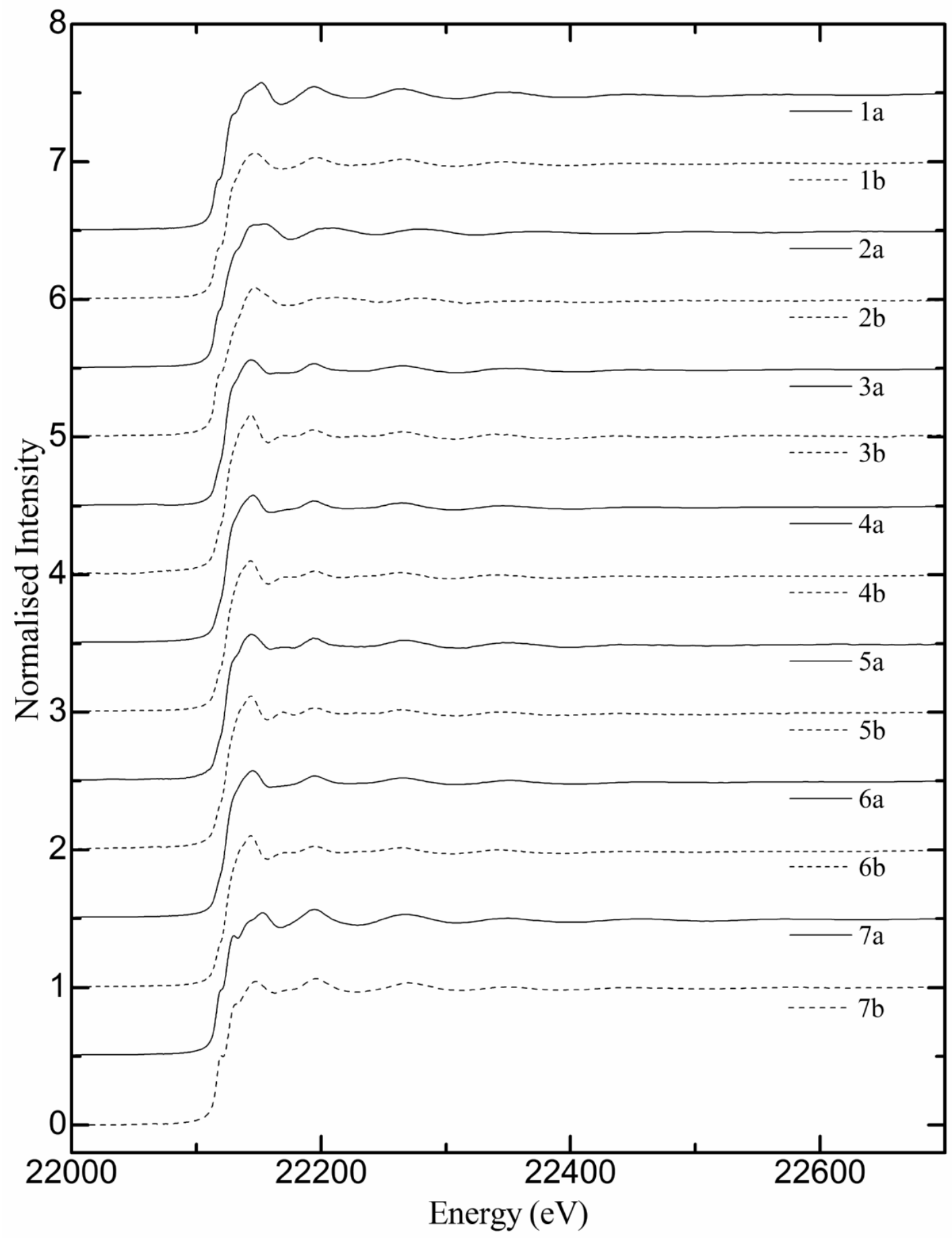


S12: Ru $L_{2,3}$ edge data for $\mathbf{1 a}, \mathbf{b}$. (A) $\mathrm{Cl} K$-edge, $R u L_{3}$-edge and $R u L_{2}$-edge XAS spectra for complexes $\mathbf{1 a}$ and $\mathbf{1 b}$ (a: $\mathrm{L}=\mathrm{PC} y_{3}, \mathbf{b}: \mathrm{L}=\mathrm{H}_{2} \mathrm{IMes}$ ). (B) Rescaled view of Ru $\mathrm{L}_{2,3}$-edges for complexes $\mathbf{1 a}$ and $\mathbf{1 b}$ (a: $\mathrm{L}=\mathrm{PC} y_{3}, \mathbf{b}$ : $\mathrm{L}=\mathrm{H}_{2} \mathrm{IMes}$ ). The spectra were normalized by a published procedure. ${ }^{9}$
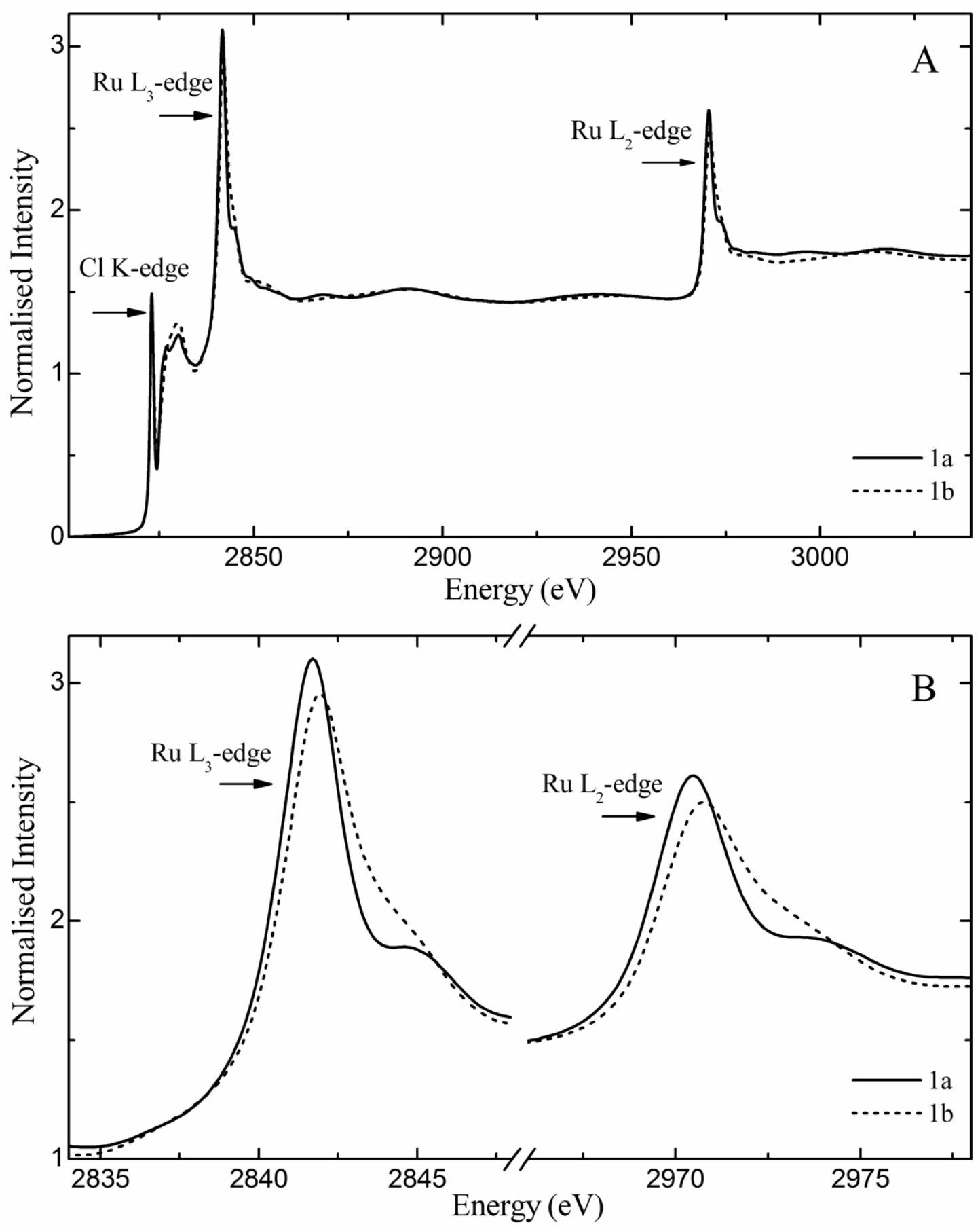
S13: Plot of $\Delta \mathrm{q}$ (averaged from Mulliken and Multipole Derived Charges) vs. $\Delta \mathrm{IE}_{1 \mathrm{~s}}$ for 3b-3a $\left(\Delta \mathrm{IE}_{1 \mathrm{~s}}=0.4 \mathrm{eV}\right) \mathbf{1} \mathbf{b}-\mathbf{1} \mathbf{a}\left(\Delta \mathrm{IE}_{1 \mathrm{~s}}=0.8 \mathrm{eV}\right)$ and $\mathbf{2 b}-\mathbf{2 a}\left(\Delta \mathrm{IE}_{1 \mathrm{~s}}=1.0\right.$ eV). See also Table 1 in the manuscript. Error bars are estimated based on multiple experimental runs and deviations between methods for calculating electron density at the metal center.

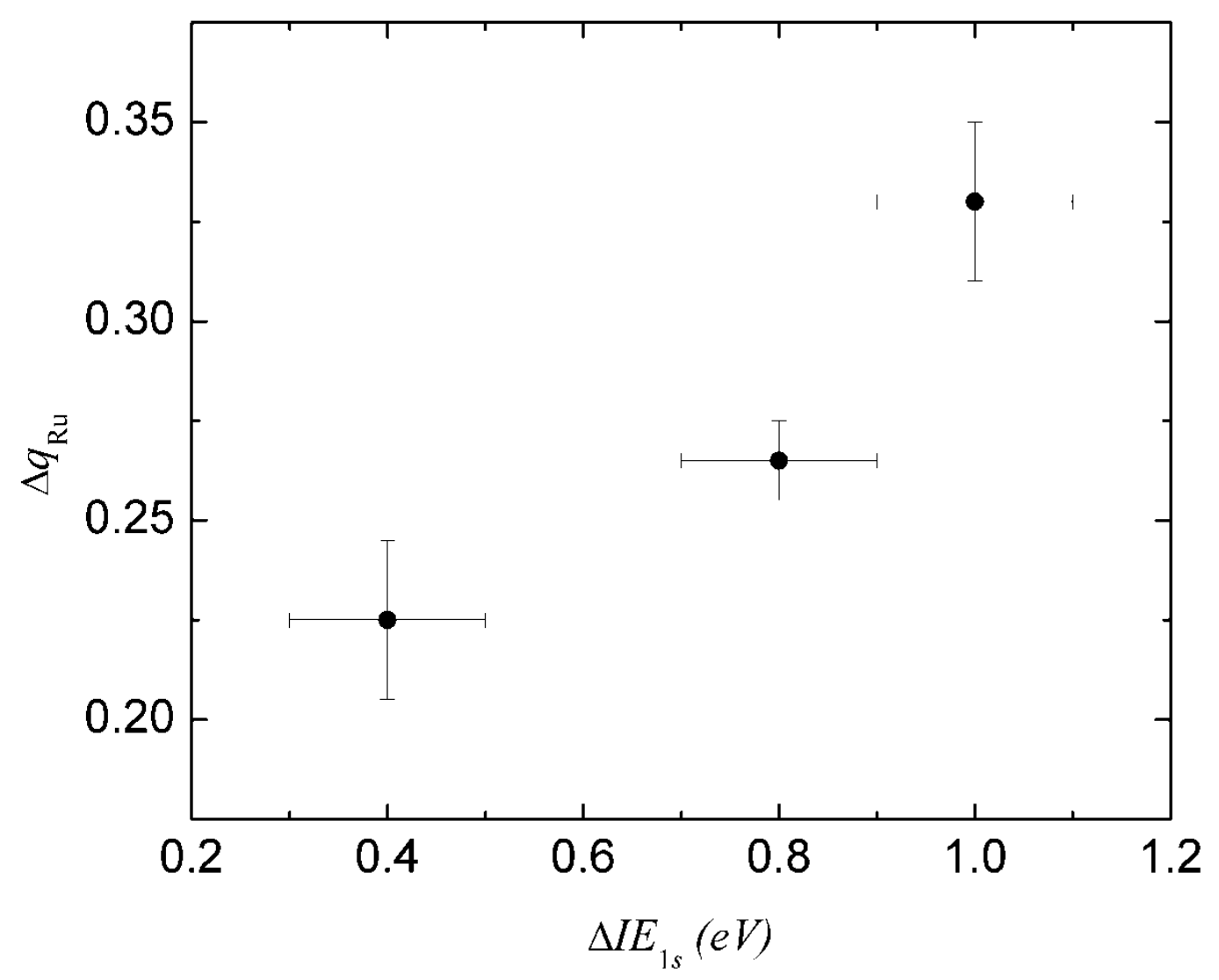


S14: Computational Details

Geometry optimizations followed by ground state DFT calculations for compounds $\mathbf{1} \mathbf{a}, \mathbf{b}, \mathbf{2} \mathbf{a}, \mathbf{b}$ and $\mathbf{3} \mathbf{a}, \mathbf{b}$ were performed using the Vosko-Wilk-Nusair local density approximation with exchange and correlation corrections from Becke $^{10}$ and Perdew ${ }^{11,12}$, respectively (BP86). Slater-type orbitals ${ }^{13}$ (STOs) were used for the triple zeta basis set with an additional set of polarization functions (TZP). The $[\mathrm{Ar}]^{18} 3 \mathrm{~d}^{10}$ core electrons of ruthenium were treated by the frozen core approximation. ${ }^{14}$ The calculations were carried out with the program package ADF2005. ${ }^{15-17}$ The run files corresponding to the ground state calculation of complexes $\mathbf{1} \mathbf{a}, \mathbf{b}, \mathbf{2} \mathbf{a}, \mathbf{b}$ and $\mathbf{3} \mathbf{a}, \mathbf{b}$ are provided below. 
S15: ADF input file for ground state calculation on first generation Grubbs catalyst (1a). Input geometry was previously optimized (Up to an integration level of 6.0) using the same density functional (Becke and Perdew) and basis set parameters (TZP). A ruthenium 3d core was used in this calculation. Complete output for this calculation will be provided upon request (pierre@chem.ubc.ca.)

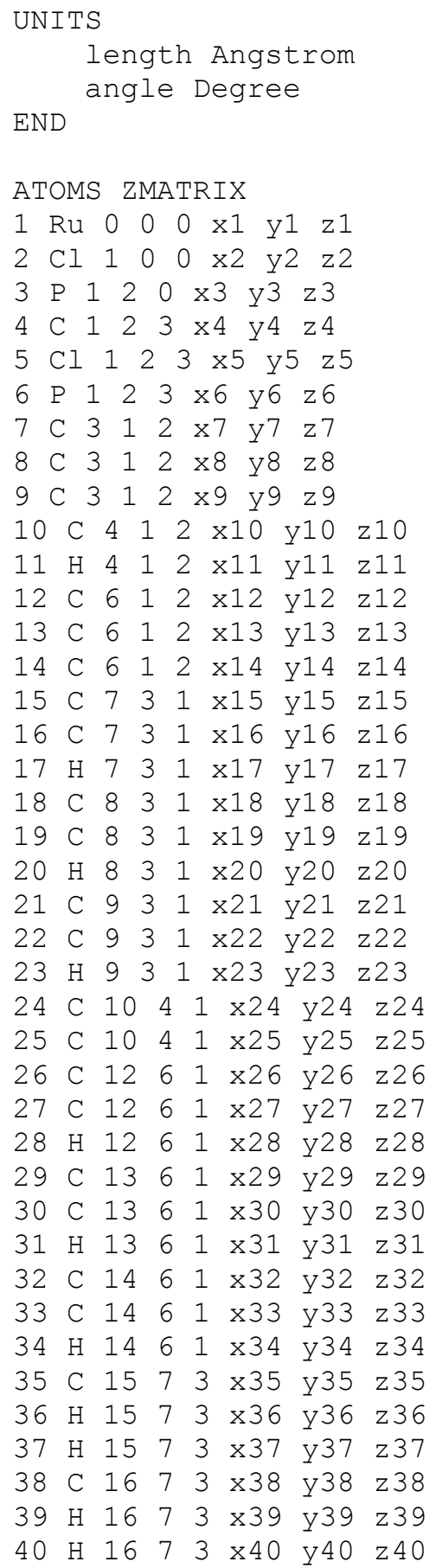


$\begin{array}{lllllllll}41 & \mathrm{C} & 18 & 8 & 3 & \mathrm{x} 41 & \mathrm{y} 41 & \mathrm{z} 41\end{array}$

$\begin{array}{lllllllll}42 & \mathrm{H} & 18 & 8 & 3 & \mathrm{x} 42 & \mathrm{y} 42 & \mathrm{z} 42\end{array}$

$\begin{array}{lllllllll}43 & \mathrm{H} & 18 & 8 & 3 & \mathrm{x} 43 & \mathrm{y} 43 & \mathrm{z} 43\end{array}$

$\begin{array}{lllllllll}44 & \mathrm{C} & 19 & 8 & 3 & \mathrm{x} 44 & \mathrm{y} 44 & \mathrm{z} 44\end{array}$

$\begin{array}{lllllllll}45 & \mathrm{H} & 19 & 8 & 3 & \mathrm{x} 45 & \mathrm{y} 45 & \mathrm{z} 45\end{array}$

46 H 1983 x46 y46 z46

$\begin{array}{lllllllll}47 & \mathrm{C} & 21 & 9 & 3 & \times 47 & \mathrm{y} 47 & \mathrm{z} 47\end{array}$

$\begin{array}{lllllllll}48 & \mathrm{H} & 21 & 9 & 3 & \mathrm{x} 48 & \text { y48 } & \mathrm{z} 48\end{array}$

$\begin{array}{lllllllll}49 & \mathrm{H} & 21 & 9 & 3 & \mathrm{x} 49 & \text { y49 } & \text { z } 49\end{array}$

$\begin{array}{llllllllll}50 & \text { C } & 22 & 9 & 3 & x 50 & \text { y50 } & \text { z } 50\end{array}$

$\begin{array}{lllllllll}51 & \mathrm{H} & 22 & 9 & 3 & \mathrm{x} 51 & \mathrm{y} 51 & \mathrm{z} 51\end{array}$

$\begin{array}{llllllll}52 & \text { H } & 22 & 9 & 3 & \text { x52 y52 z52 }\end{array}$

53 C $24 \quad 10 \quad 4 \quad x 53$ y53 z53

$\begin{array}{lllllllll}54 & \mathrm{H} & 24 & 10 & 4 & x 54 & \text { y54 } & \text { z5 } 4\end{array}$

$\begin{array}{llllllll}55 & \mathrm{C} & 25 & 10 & 4 & \mathrm{x} 55 & \mathrm{y} 55 & \mathrm{z} 55\end{array}$

$\begin{array}{lllllllll}56 & \mathrm{H} & 25 & 10 & 4 & \mathrm{x} 56 & \text { y5 } & \mathrm{z} 56\end{array}$

$\begin{array}{llllllll}57 & \mathrm{C} & 26 & 12 & 6 & \mathrm{x} 57 & \mathrm{y} 57 & \mathrm{z} 57\end{array}$

$\begin{array}{lllllllll}58 & \mathrm{H} & 26 & 12 & 6 & \mathrm{x} 58 & \text { y58 } & \mathrm{z} 58\end{array}$

$\begin{array}{llllllll}59 & \mathrm{H} & 26 & 12 & 6 & \times 59 & \text { y59 z59 }\end{array}$

$\begin{array}{lllllllll}60 & \mathrm{C} & 27 & 12 & 6 & \mathrm{x} 60 & \text { y } 60 & \mathrm{z} 60\end{array}$

$\begin{array}{lllllllll}61 & \mathrm{H} & 27 & 12 & 6 & \mathrm{x} 61 & \mathrm{y} 61 & \mathrm{z} 61\end{array}$

$\begin{array}{llllllll}62 & \mathrm{H} & 27 & 12 & 6 & \mathrm{x} 62 & \mathrm{y} 62 & \mathrm{z} 62\end{array}$

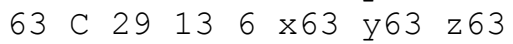

$\begin{array}{lllllllll}64 & \mathrm{H} & 29 & 13 & 6 & \mathrm{x} 64 & \mathrm{y} 64 & \mathrm{z} 64\end{array}$

$\begin{array}{llllllll}65 & \mathrm{H} & 29 & 13 & 6 & \mathrm{x} 65 & \text { y } 65 & \mathrm{z} 65\end{array}$

66 C $3013 \quad 6$ x66 y66 z66

$\begin{array}{lllllllll}67 & \mathrm{H} & 30 & 13 & 6 & \mathrm{x} 67 & \text { y } 67 & \mathrm{z} 67\end{array}$

$\begin{array}{llllllll}68 & \mathrm{H} & 30 & 13 & 6 & \times 68 & \text { y68 } & \text { z68 }\end{array}$

69 C $3214 \quad 6 \quad x 69$ y69 z69

$\begin{array}{lllllllll}70 & \mathrm{H} & 32 & 14 & 6 & \mathrm{x} 70 & \text { y70 } & \text { z } 70\end{array}$

$\begin{array}{llllllll}71 & \mathrm{H} & 32 & 14 & 6 & \mathrm{x} 71 & \mathrm{y} 71 & \mathrm{z} 71\end{array}$

$\begin{array}{lllllllll}72 & \mathrm{C} & 33 & 14 & 6 & \mathrm{x} 72 & \mathrm{y} 72 & \mathrm{z} 72\end{array}$

$\begin{array}{lllllllll}73 & \mathrm{H} & 33 & 14 & 6 & \mathrm{x} 73 & \mathrm{y} 73 & \mathrm{z} 73\end{array}$

$\begin{array}{llllllll}74 & \mathrm{H} & 33 & 14 & 6 & \mathrm{x} 74 & \mathrm{y} 74 & \mathrm{z} 74\end{array}$

$\begin{array}{llllllll}75 & \mathrm{C} & 35 & 15 & 7 & \mathrm{x} 75 & \mathrm{y} 75 & \mathrm{z} 75\end{array}$

$\begin{array}{lllllllll}76 & \mathrm{H} & 35 & 15 & 7 & \mathrm{x} 76 & \mathrm{y} 76 & \mathrm{z} 76\end{array}$

$\begin{array}{lllllllll}77 & \mathrm{H} & 35 & 15 & 7 & \mathrm{x} 77 & \mathrm{y} 77 & \mathrm{z} 77\end{array}$

$\begin{array}{lllllllll}78 & \mathrm{H} & 38 & 16 & 7 & \mathrm{x} 78 & \mathrm{y} 78 & \mathrm{z} 78\end{array}$

$\begin{array}{lllllllll}79 & \mathrm{H} & 38 & 16 & 7 & \mathrm{x} 79 & \mathrm{y} 79 & \mathrm{z} 79\end{array}$

80 C $41 \quad 18 \quad 8 \quad x 80$ y80 z80

$\begin{array}{lllllllll}81 & \mathrm{H} & 41 & 18 & 8 & \mathrm{x} 81 & \mathrm{y} 81 & \mathrm{z} 81\end{array}$

$\begin{array}{lllllllll}82 & \mathrm{H} & 41 & 18 & 8 & \mathrm{x} 82 & \mathrm{y} 82 & \mathrm{z} 82\end{array}$

$\begin{array}{lllllllll}83 & \mathrm{H} & 44 & 19 & 8 & \mathrm{x} 83 & \text { y83 } & \mathrm{z} 83\end{array}$

$\begin{array}{lllllllll}84 & \mathrm{H} & 44 & 19 & 8 & \mathrm{x} 84 & \mathrm{y} 84 & \mathrm{z} 84\end{array}$

$\begin{array}{lllllllll}85 & \mathrm{C} & 47 & 21 & 9 & \mathrm{x} 85 & \text { y85 } & \mathrm{z} 85\end{array}$

$\begin{array}{lllllllll}86 & \mathrm{H} & 47 & 21 & 9 & \mathrm{x} 86 & \text { y86 } & \text { z86 }\end{array}$

$\begin{array}{lllllllll}87 & \mathrm{H} & 47 & 21 & 9 & \mathrm{x} 87 & \mathrm{y} 87 & \mathrm{z} 87\end{array}$

$\begin{array}{lllllllll}88 & \mathrm{H} & 50 & 22 & 9 & \mathrm{x} 88 & \text { y88 } & \mathrm{z} 88\end{array}$

$89 \mathrm{H} \quad 50 \quad 22 \quad 9 \times 89$ y89 z89

90 C $5324 \quad 10 \times 90$ y90 z90

$\begin{array}{lllllllll}91 & \mathrm{H} & 53 & 24 & 10 & \mathrm{x} 91 & \mathrm{y} 91 & \mathrm{z} 91\end{array}$

$\begin{array}{llllllll}92 & \mathrm{H} & 55 & 25 & 10 & x 92 & \text { y92 } & \text { z } 92\end{array}$

93 C $57 \quad 26 \quad 12 \times 93$ y93 z93

$\begin{array}{lllllllll}94 & \mathrm{H} & 57 & 26 & 12 & \times 94 & \text { y } 94 & \mathrm{z} 94\end{array}$

$\begin{array}{lllllllll}95 & \mathrm{H} & 57 & 26 & 12 & \text { x95 } & \text { y95 } & \mathrm{z} 95\end{array}$

$\begin{array}{lllllllll}96 & \mathrm{H} & 60 & 27 & 12 & \text { x96 } & \text { y96 } & \text { z } 96\end{array}$

$\begin{array}{llllllll}97 & \mathrm{H} & 60 & 27 & 12 & \text { x97 } & \text { y97 } & \text { z97 }\end{array}$ 
$\begin{array}{lllllllll}98 & \mathrm{C} & 63 & 29 & 13 & \times 98 & \text { y98 z } 98\end{array}$

99 H $63 \quad 29 \quad 13 \times 99$ y99 z99

100 H $63 \quad 29 \quad 13 \times 100$ y100 z100

$101 \mathrm{H} \quad 66 \quad 30 \quad 13 \times 101$ y101 z101

102 H $66 \quad 30 \quad 13 \times 102 \quad y 102 \quad z 102$

103 C 693214 x103 y103 z103

$\begin{array}{lllllllll}104 & \mathrm{H} & 69 & 32 & 14 & \mathrm{x} 104 & \mathrm{y} 104 & \mathrm{z} 104\end{array}$

$\begin{array}{llllllll}105 & \mathrm{H} & 69 & 32 & 14 & \mathrm{x} 105 & \mathrm{y} 105 & \mathrm{z} 105\end{array}$

$106 \mathrm{H} 72 \quad 33 \quad 14$ x106 y106 z106

$107 \mathrm{H} 72 \quad 33 \quad 14 \times 107$ y107 z107

$108 \mathrm{H} \quad 75 \quad 35 \quad 15 \times 108$ y108 z108

$109 \mathrm{H} 75 \quad 35 \quad 15 \times 109$ y109 z109

$\begin{array}{lllllllll}110 & \mathrm{H} & 80 & 41 & 18 & \mathrm{x} 110 & \mathrm{y} 110 & \mathrm{z} 110\end{array}$

$\begin{array}{lllllllll}111 & \mathrm{H} & 80 & 41 & 18 & \mathrm{x} 111 & \mathrm{y} 111 & \mathrm{z} 111\end{array}$

$\begin{array}{llllllll}112 & \mathrm{H} & 85 & 47 & 21 & \mathrm{x} 112 & \mathrm{y} 112 & \mathrm{z} 112\end{array}$

$\begin{array}{lllllllll}113 & \mathrm{H} & 85 & 47 & 21 & \mathrm{x} 113 & \mathrm{y} 113 & \mathrm{z} 113\end{array}$

$\begin{array}{llllllll}114 & \mathrm{H} & 90 & 53 & 24 & \mathrm{x} 114 & \mathrm{y} 114 & \mathrm{z} 114\end{array}$

$\begin{array}{llllllll}115 & \mathrm{H} & 93 & 57 & 26 & \mathrm{x} 115 & \mathrm{y} 115 & \mathrm{z} 115\end{array}$

$\begin{array}{llllllll}116 \mathrm{H} & 93 & 57 & 26 & \mathrm{x} 116 & \mathrm{y} 116 & \mathrm{z} 116\end{array}$

$\begin{array}{lllllllll}117 & \mathrm{H} & 98 & 63 & 29 & \mathrm{x} 117 & \mathrm{y} 117 & \mathrm{z} 117\end{array}$

$\begin{array}{llllllll}118 & \mathrm{H} & 98 & 63 & 29 & \mathrm{x} 118 & \mathrm{y} 118 & \mathrm{z} 118\end{array}$

119 H $103 \quad 69 \quad 32 \times 119$ y119 z119

$120 \mathrm{H} \quad 103 \quad 69 \quad 32 \times 120$ y120 z120

END

GEOVAR

$x 10.0$

y1 0.0

$\mathrm{z} 10.0$

$\times 22.49075345322$

y2 0.0

z2 0.0

$\times 32.50215152595$

y3 89.5912914233

z3 0.0

x4 1.8803837697

y4 106.883936795

z4 265.860102596

$\times 52.48017811496$

y5 161.654908674

z5 95.6771027599

$\times 62.51539611862$

y6 89.4047056936

z6 163.313295541

$\times 71.91303056806$

y7 118.364121634

z7 176.637302297

x8 1.89988474214

y8 120.870056971

z8 300.903243509

x91.9044042173

y9 110.995586292

z9 60.347188

$\times 101.4595639932$

y10 135.6053711

z10 347.054134399

x11 1.10703633077 
y11 112.752164148

z11 167.637528679

x12 1.90405220629

y12 115.541569987

z12 155.116119282

x131.88345568139

y13 114.855430374

z13 24.106312401

x14 1.88985714544

y14 103.023065658

z14 267.190565672

$\times 151.56314523075$

y15 113.020685865

z15 50.0934339904

x16 1.54318956337

y16 114.654153826

z16 283.141323159

x17 1.10736282865

y17 102.524653492

z17 167.192608086

x18 1.54148638509

y18 114.842027399

z18 41.6122587112

x191.55388053158

y19 114.219873905

z19 275.359138925

x20 1.10661476164

y20 102.465991542

z20 158.956600149

x21 1.54193063027

y21 117.391027703

z21 265.901359585

x22 1.55483181025

y22 110.746689853

z22 31.9290393932

x231.1060420967

y23 103.209713091

z23 148.583647857

x241.41633980248

y24 117.09075787

z24 185.237766898

×251.41449682673

y25 124.79097559

z25 5.66585169785

x261.54117469915

y26 114.678655205

z26 321.739969494

x27 1.5562279601

y27 115.945750141

z27 193.05920786

x281.10475940923

y28 100.581407825

z28 75.3078835697

x291.55654027042

y29 119.165296145

z29 205.403358107

$\times 301.54448350572$ 
y30 111.872937378

z30 74.3931515584

×31 1.10680048184

y31 99.6555069499

z31 322.606668372

$\times 321.5439663587$

y32 113.161392971

z32 68.4888344783

x33 1.55595619004

y33 117.319227941

z33 197.917912316

×341.10704913095

y34 100.300516257

z34 314.075420613

x35 1.54203621473

y35 112.938324344

z35 210.462119026

×36 1.09935396455

y36 109.27814543

z36 89.1720995819

x37 1.09810306109

y37 109.807895026

z37 331.578858231

×38 1.54741378672

y38 111.264002295

z38 190.402692545

×391.09986513721

y39 108.307010556

z39 68.7996134871

$\times 401.09813103865$

y40 111.468149315

z40 311.350493875

$\times 411.5458248125$

y41 110.085757139

z41 162.863487598

x42 1.09490552763

y42 112.751871523

z42 38.5868180735

$x 431.10032543627$

y43 107.898860902

z43 282.95381023

x441.55066954669

y44 112.170460809

z44 161.447650852

x45 1.10109344884

y45 110.146734261

z45 40.1688609127

x461.10066271429

y46 109.394648422

z46 282.97191064

x47 1.55092338917

y47 110.100107806

z47 193.34653253

x48 1.10082856722

y48 108.711687867

z48 71.9125510167

×491.097415242 
y49 111.873803033

z49 314.336618904

x50 1.54931038303

y50 112.525933744

z50 196.670213503

x51 1.09933796246

y51 109.933135408

z51 73.2746063975

x52 1.10251473879

y52 109.029176165

z52 317.191247217

x53 1.39308633523

y53 121.232444969

z53 179.771600315

x54 1.09134483316

y54 118.695337929

z54 0.036189622384

x55 1.39260267866

y55 120.461436507

z55 179.625263225

x56 1.09239917123

y56 118.338097771

z56 0.29229008499

$\times 571.54800136635$

y57 110.145970265

z57 161.415133394

$x 581.0962160189$

y58 110.216588469

z58 40.9152274707

$\times 591.10273813699$

y59 109.517229031

z59 282.994843576

x60 1.55109701818

y60 112.222507658

z60 162.518445332

x61 1.09772858079

y61 110.572469816

z61 41.3743302978

$\mathrm{x} 621.09870207219$

y62 109.476209863

z62 284.095982022

x63 1.54979209887

y63 111.591441431

z63 199.152344983

x64 1.10094227114

y64 108.876478218

z64 78.5025770641

x65 1.0973472016

y65 111.908438782

z65 321.212794978

х66 1.54772035739

y66 110.227877968

z66 200.652206182

x67 1.10252081043

y67 109.647644038

z67 79.5674017377

x68 1.09950964728 


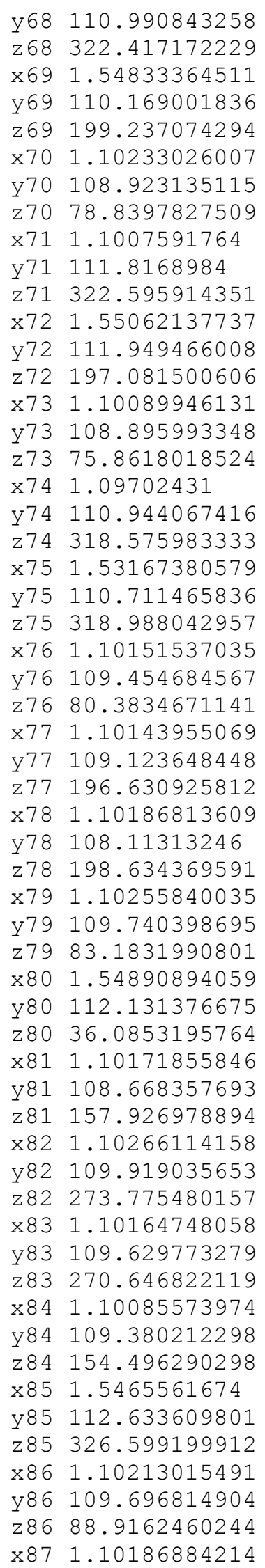




$\begin{array}{ll}\text { y87 } & 108.557678763 \\ \text { z87 } & 204.691553932 \\ \text { x88 } & 1.10016526588 \\ \text { y88 } & 109.760065497 \\ \text { z88 } & 206.835564694 \\ \text { x89 } & 1.10206664437 \\ \text { y89 } & 109.636478576 \\ \text { z89 } & 90.4126492836 \\ \text { x90 } & 1.39940375698 \\ \text { y90 } & 119.800920249 \\ \text { z90 } & 0.645386399026 \\ \text { x91 } & 1.09103658563 \\ \text { y91 } & 119.99138543 \\ \text { z91 } & 180.477044875 \\ \text { x92 } & 1.09135962482 \\ \text { y92 } & 119.631529407 \\ \text { z92 } & 180.352351506 \\ \text { x93 } & 1.54838963997 \\ \text { y93 } & 112.52190486 \\ \text { z93 } & 34.8801606294 \\ \text { x94 } & 1.10204263326 \\ \text { y94 } & 108.836243883 \\ \text { z94 } & 156.895677634 \\ \text { x95 } & 1.10077198833 \\ \text { y95 } & 109.071781381 \\ \text { z95 } & 272.896950136 \\ \text { x96 } & 1.10264437387 \\ \text { y96 } & 109.762164289 \\ \text { z96 } & 271.192905208 \\ \text { x97 } & 1.10111054778 \\ \text { y97 } & 109.362500511 \\ \text { z97 } & 155.028589428 \\ \text { x98 } & 1.53341597838 \\ \text { y98 } & 111.190272791 \\ \text { z98 } & 326.650561483 \\ \text { x99 } & 1.10330667812 \\ \text { y99 } & 109.835682156 \\ \text { z99 } & 87.3450044826 \\ \text { x100 } & 1.1010532893 \\ \text { y100 } & 109.461388464 \\ \text { z100 } & 203.526484153 \\ \text { x101 } & 1.10194733303 \\ \text { y101 } & 108.947217416 \\ \text { z101 } & 203.534911087 \\ \text { x1002 } & 1.1004749282 \\ \text { y102 } & 109.331494512 \\ \text { z102 } & 87.348337083 \\ \text { x103 } & 1.54811297096 \\ \text { y103 } & 112.419863453 \\ \text { z103 } & 326.894382175 \\ \text { x104 } & 1.09986650055 \\ \text { y104 } & 109.564497392 \\ \text { z104 } & 88.7387168943 \\ \text { x105 } & 1.1019275479 \\ \text { y105 } & 108.899458888 \\ \text { z105 } & 204.752531647 \\ \text { x106 } & 1.10083431377\end{array}$




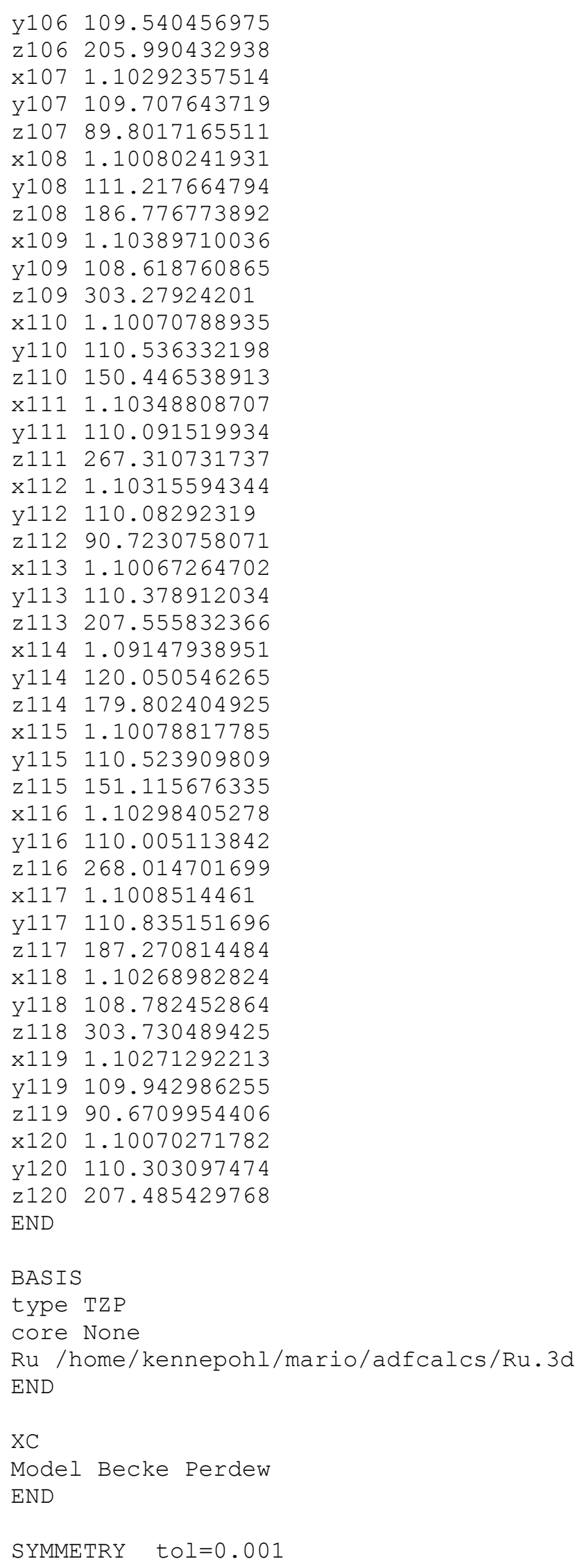




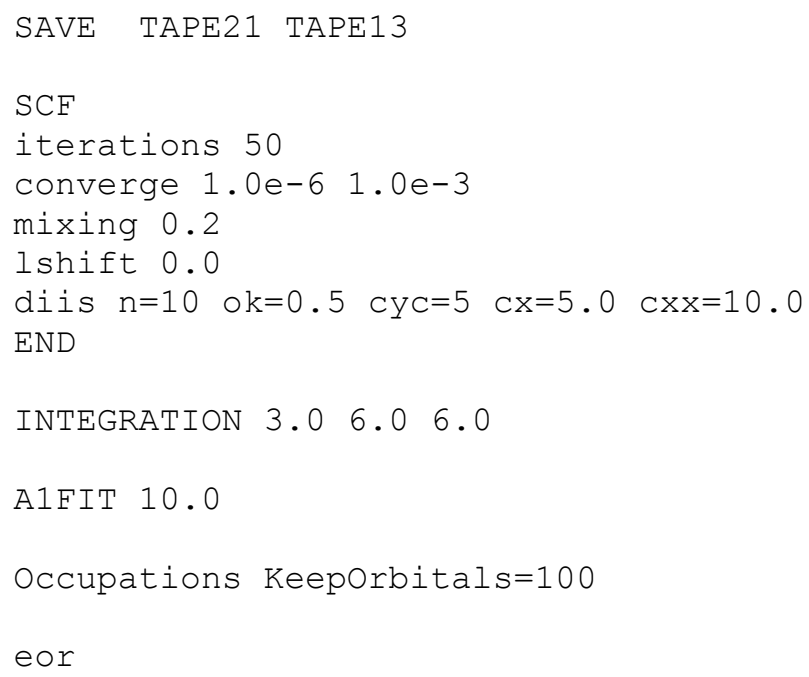


S16: ADF input file for ground state calculation on second generation Grubbs catalyst (1b). Input geometry was previously optimized (Up to an integration level of 6.0) using the same density functional (Becke and Perdew) and basis set parameters (TZP). A ruthenium 3d core was used in this calculation. Complete output for this calculation will be provided upon request (pierre@chem.ubc.ca.)

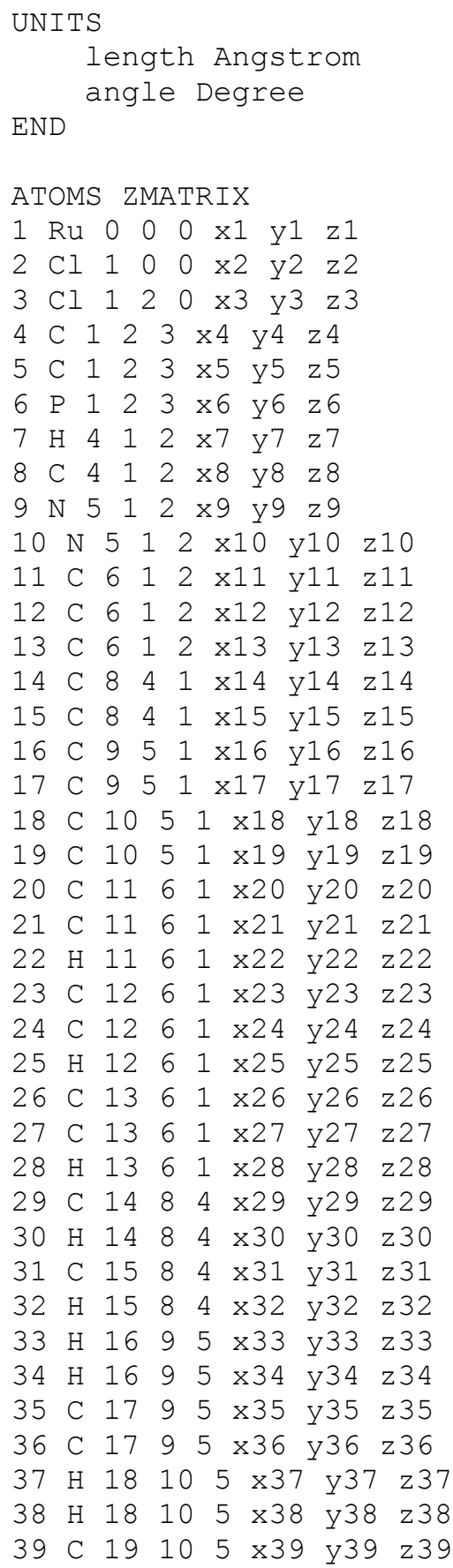


$\begin{array}{lllllllll}40 & C & 19 & 10 & 5 & x 40 & \text { y } 40 & \text { z } 40\end{array}$

$\begin{array}{lllllllll}41 & \mathrm{C} & 20 & 11 & 6 & \mathrm{x} 41 & \mathrm{y} 41 & \mathrm{z} 41\end{array}$

$\begin{array}{lllllllll}42 & \text { H } & 20 & 11 & 6 & x 42 & y 42 & z 42\end{array}$

$\begin{array}{lllllllll}43 & \mathrm{H} & 20 & 11 & 6 & \mathrm{x} 43 & \mathrm{y} 43 & \mathrm{z} 43\end{array}$

$\begin{array}{llllllll}44 & \mathrm{C} & 21 & 11 & 6 & \mathrm{x} 44 & \mathrm{y} 44 & \mathrm{z} 44\end{array}$

$\begin{array}{llllllll}45 & \mathrm{H} & 21 & 11 & 6 & \mathrm{x} 45 & \mathrm{y} 45 & \mathrm{z} 45\end{array}$

$\begin{array}{llllllll}46 & \mathrm{H} & 21 & 11 & 6 & \mathrm{x} 46 & \mathrm{y} 46 & \mathrm{z} 46\end{array}$

$\begin{array}{llllllll}47 & \mathrm{C} & 23 & 12 & 6 & \mathrm{x} 47 & \mathrm{y} 47 & \mathrm{z} 47\end{array}$

$\begin{array}{lllllllll}48 & \mathrm{H} & 23 & 12 & 6 & \mathrm{x} 48 & \mathrm{y} 48 & \mathrm{z} 48\end{array}$

$\begin{array}{llllllll}49 & \mathrm{H} & 23 & 12 & 6 & \mathrm{x} 49 & \text { y49 } & \mathrm{z} 49\end{array}$

$\begin{array}{llllllll}50 & \mathrm{C} & 24 & 12 & 6 & \times 50 & \text { y50 } & \mathrm{z} 50\end{array}$

$\begin{array}{lllllllll}51 & \mathrm{H} & 24 & 12 & 6 & \mathrm{x} 51 & \mathrm{y} 51 & \mathrm{z} 51\end{array}$

$\begin{array}{llllllll}52 & \mathrm{H} & 24 & 12 & 6 & \mathrm{x} 52 & \mathrm{y} 52 & \mathrm{z} 52\end{array}$

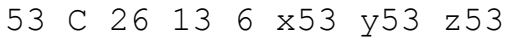

$\begin{array}{llllllll}54 & \mathrm{H} & 26 & 13 & 6 & \mathrm{x} 54 & \mathrm{y} 54 & \mathrm{z} 54\end{array}$

$\begin{array}{llllllll}55 & \mathrm{H} & 26 & 13 & 6 & \mathrm{x} 55 & \text { y55 } & \text { z55 }\end{array}$

$\begin{array}{lllllllll}56 & \mathrm{C} & 27 & 13 & 6 & \mathrm{x} 56 & \text { y5 } & \mathrm{z} 56\end{array}$

$\begin{array}{lllllllll}57 & \mathrm{H} & 27 & 13 & 6 & \mathrm{x} 57 & \mathrm{y} 57 & \mathrm{z} 57\end{array}$

$\begin{array}{lllllllll}58 & \mathrm{H} & 27 & 13 & 6 & \times 58 & \text { y58 } & \mathrm{z} 58\end{array}$

$\begin{array}{lllllll}59 & \text { C } & 29 & 14 & 8 & x 59 & \text { y59 z59 }\end{array}$

$\begin{array}{llllllllll}60 & \mathrm{H} & 29 & 14 & 8 & \mathrm{x} 60 & \text { y } 60 & \text { z } 60\end{array}$

$\begin{array}{lllllllll}61 & \mathrm{H} & 31 & 15 & 8 & \mathrm{x} 61 & \mathrm{y} 61 & \mathrm{z} 61\end{array}$

$\begin{array}{llllllll}62 & \mathrm{C} & 35 & 17 & 9 & \mathrm{x} 62 & \mathrm{y} 62 & \mathrm{z} 62\end{array}$

63 C $35179 \times 63$ y63 z63

$\begin{array}{lllllllll}64 & \mathrm{C} & 36 & 17 & 9 & \mathrm{x} 64 & \mathrm{y} 64 & \mathrm{z} 64\end{array}$

65 C 36179 x65 y65 z65

$\begin{array}{llllllll}66 & \text { C } & 39 & 19 & 10 & \text { x66 y66 z } 66\end{array}$

$\begin{array}{lllllllll}67 & \mathrm{C} & 39 & 19 & 10 & \mathrm{x} 67 & \mathrm{y} 67 & \mathrm{z} 67\end{array}$

$\begin{array}{lllllllll}68 & \mathrm{C} & 40 & 19 & 10 & \text { x68 } & \text { y68 } & \text { z } 68\end{array}$

69 C $40 \quad 19 \quad 10 \quad x 69$ y69 z69

$\begin{array}{llllllllll}70 & C & 41 & 20 & 11 & x 70 & y 70 & z 70\end{array}$

$\begin{array}{lllllllll}71 & \mathrm{H} & 41 & 20 & 11 & x 71 & y 71 & \mathrm{z} 71\end{array}$

$\begin{array}{llllllll}72 & \text { H } & 41 & 20 & 11 & x 72 & y 72 & \text { z } 72\end{array}$

$\begin{array}{llllllll}73 & \mathrm{H} & 44 & 21 & 11 & \mathrm{x} 73 & \mathrm{y} 73 & \mathrm{z} 73\end{array}$

$\begin{array}{llllllll}74 & \mathrm{H} & 44 & 21 & 11 & \mathrm{x} 74 & \mathrm{y} 74 & \mathrm{z} 74\end{array}$

$\begin{array}{llllllll}75 & C & 47 & 23 & 12 & x 75 & y 75 & z 75\end{array}$

$\begin{array}{lllllllll}76 & \mathrm{H} & 47 & 23 & 12 & \mathrm{x} 76 & \text { y76 } & \mathrm{z} 76\end{array}$

$\begin{array}{lllllllll}77 & \mathrm{H} & 47 & 23 & 12 & \mathrm{x} 77 & \mathrm{y} 77 & \mathrm{z} 77\end{array}$

$\begin{array}{llllllll}78 & \mathrm{H} & 50 & 24 & 12 & \mathrm{x} 78 & \mathrm{y} 78 & \mathrm{z} 78\end{array}$

$\begin{array}{lllllllll}79 & \mathrm{H} & 50 & 24 & 12 & \text { x79 } & \text { y79 } & \text { z } 79\end{array}$

$80 \quad C \quad 53 \quad 26 \quad 13 \quad x 80 \quad y 80 \quad$ z 80

$81 \mathrm{H} \quad 53 \quad 26 \quad 13$ x81 y81 z81

$\begin{array}{llllllll}82 & \mathrm{H} & 53 & 26 & 13 & \mathrm{x} 82 & \text { y82 } & \mathrm{z} 82\end{array}$

$\begin{array}{lllllllll}83 & \mathrm{H} & 56 & 27 & 13 & \mathrm{x} 83 & \mathrm{y} 83 & \mathrm{z} 83\end{array}$

$\begin{array}{lllllllll}84 & \mathrm{H} & 56 & 27 & 13 & \mathrm{x} 84 & \mathrm{y} 84 & \mathrm{z} 84\end{array}$

$\begin{array}{llllllll}85 & \mathrm{H} & 59 & 29 & 14 & x 85 & \text { y8 } 8 & \text { z } 85\end{array}$

86 C $62 \quad 35 \quad 17 \times 86 \quad$ y86 z86

$\begin{array}{lllllllll}87 & \mathrm{H} & 62 & 35 & 17 & \mathrm{x} 87 & \mathrm{y} 87 & \mathrm{z} 87\end{array}$

$\begin{array}{lllllllll}88 & \mathrm{H} & 63 & 35 & 17 & \mathrm{x} 88 & \mathrm{y} 88 & \mathrm{z} 88\end{array}$

$\begin{array}{llllllllll}89 & \mathrm{H} & 63 & 35 & 17 & \times 89 & \text { y89 } & \text { z } 89\end{array}$

$\begin{array}{llllllllll}90 & \mathrm{H} & 63 & 35 & 17 & \times 90 & \text { y90 } & \text { z } 90\end{array}$

$\begin{array}{lllllllll}91 & \mathrm{H} & 64 & 36 & 17 & x 91 & y 91 & \mathrm{z} 91\end{array}$

$\begin{array}{lllllllll}92 & \mathrm{H} & 65 & 36 & 17 & \text { x92 } & \text { y92 } & \text { z92 }\end{array}$

$\begin{array}{llllllll}93 & \mathrm{H} & 65 & 36 & 17 & \text { x93 y93 z93 }\end{array}$

$\begin{array}{lllllllll}94 & \mathrm{H} & 65 & 36 & 17 & \times 94 & \text { y94 } & \mathrm{z} 94\end{array}$

95 C $66 \quad 39 \quad 19 \times 95$ y95 z95

96 H $66 \quad 39 \quad 19 \times 96$ y96 z96 
$\begin{array}{lllllllll}97 & \mathrm{H} & 67 & 39 & 19 & \times 97 & \text { y97 } & \text { z } 97\end{array}$

$\begin{array}{lllllllll}98 & \mathrm{H} & 67 & 39 & 19 & \times 98 & \text { y98 } & \text { z } 98\end{array}$

99 H $67 \quad 39 \quad 19 \times 99 \quad$ y99 z99

100 H $68 \quad 40 \quad 19 \times 100$ y100 z100

101 H $6940 \quad 19 \times 101$ y101 z101

$102 \mathrm{H} \quad 6940 \quad 19 \times 102$ y102 z102

$103 \mathrm{H} \quad 6940 \quad 19 \times 103$ y103 z103

$\begin{array}{llllllll}104 \mathrm{H} & 70 & 41 & 20 & \mathrm{x} 104 & \mathrm{y} 104 & \mathrm{z} 104\end{array}$

$\begin{array}{llllllll}105 & \mathrm{H} & 70 & 41 & 20 & \mathrm{x} 105 & \text { y105 z105 }\end{array}$

$106 \mathrm{H} 754723 \times 106$ y106 z106

$\begin{array}{lllllllll}107 & \mathrm{H} & 75 & 47 & 23 & \mathrm{x} 107 & \mathrm{y} 107 & \mathrm{z} 107\end{array}$

$\begin{array}{llllllll}108 & \mathrm{H} & 80 & 53 & 26 & \mathrm{x} 108 & \mathrm{y} 108 & \mathrm{z} 108\end{array}$

$109 \mathrm{H} \quad 80 \quad 53 \quad 26 \times 109$ y109 z109

110 C $86 \quad 62 \quad 35$ x110 y110 z110

111 C $95 \quad 66 \quad 39 \times 111$ y111 z111

$112 \mathrm{H} 110 \quad 86 \quad 62 \times 112$ y112 z112

$\begin{array}{llllllll}113 & \mathrm{H} & 110 & 86 & 62 & \mathrm{x} 113 & \mathrm{y} 113 & \mathrm{z} 113\end{array}$

$\begin{array}{llllllll}114 & \mathrm{H} & 110 & 86 & 62 & \mathrm{x} 114 & \mathrm{y} 114 & \mathrm{z} 114\end{array}$

$\begin{array}{llllllll}115 & \mathrm{H} & 111 & 95 & 66 & \mathrm{x} 115 & \mathrm{y} 115 & \mathrm{z} 115\end{array}$

$116 \mathrm{H} \quad 1119566 \times 116$ y116 z116

$\begin{array}{llllllllll}117 & \mathrm{H} & 111 & 95 & 66 & \mathrm{x} 117 & \mathrm{y} 117 & \mathrm{z} 117\end{array}$

END

GEOVAR

$x 10.0$

$y 10.0$

z 10.0

$x 22.485619967$

y2 0.0

z2 0.0

×3 2.49962200316

y3 170.375532386

z3 0.0

$\times 41.88360466417$

y4 102.079371676

z4 190.109385337

$\times 5 \quad 2.12760989978$

y5 89.718514933

z5 88.2938780118

$\times 62.51718464645$

y6 89.4029424624

z6 287.02387711

x7 1.10469764386

y7 111.019090506

z7 185.522674605

x8 1.46185842656

y8 137.574462323

z8 8.29233799408

$\times 91.35981872948$

y9 128.504782344

z9 99.7405298021

x10 1.3639515305

y10 124.468474511

z10 278.248214077

x11 1.89618263138

y11 114.662841451

z11 331.376538844 
x12 1.88740293426

y12 110.145371057

z12 86.8186611339

x131.8860936039

y13 115.87930949

z13 199.907601675

x14 1.41686046252

y14 116.334501326

z14 172.569946525

x15 1.41407536481

y15 125.869247712

z15 352.700160562

x16 1.48282307855

y16 113.38963234

z16 175.665320237

x17 1.43982186667

y17 130.870827295

z17 356.139858493

x18 1.48291264995

y18 113.309329115

z18 178.25945535

x191.44383490681

y19 130.495093439

z19 357.688401113

x20 1.55066247268

y20 112.67453525

z20 276.227538522

x211.54236081946

y21 119.566085977

z21 146.168301661

x22 1.10704061001

y22 99.5946023447

z22 30.0681661378

x231.54199490694

y23 112.993575846

z23 304.426011452

x241.55354921113

y24 113.988984957

z24 70.7491192134

x251.10431629883

y25 103.904176515

z25 187.874411063

x261.54047031967

y26 116.875305166

z26 51.3168129729

x27 1.55012069536

y27 114.421357889

z27 182.509553807

×281.1051247156

y28 99.7763510776

z28 298.487080487

x291.39344059909

y29121.405910147

z29 180.60147835

x301.09112240123

y30 118.642493293

z30 0.324513582628 


$\begin{array}{ll}\text { x31 } & 1.39293266281 \\ \text { y31 } & 120.657848555 \\ \text { z31 } & 180.045374714 \\ \text { x32 } & 1.09101048443 \\ \text { y32 } & 118.378815894 \\ \text { z32 } & 0.111276150948 \\ \text { x33 } & 1.09802725266 \\ \text { y33 } & 110.169452031 \\ \text { z33 } & 128.130894165 \\ \text { x34 } & 1.10047934913 \\ \text { y34 } & 109.900498317 \\ \text { z34 } & 247.082820534 \\ \text { x35 } & 1.41122460365 \\ \text { y35 } & 118.43247515 \\ \text { z35 } & 266.564690459 \\ \text { x36 } & 1.40867345465 \\ \text { y36 } & 119.500734708 \\ \text { z36 } & 95.5230426308 \\ \text { x37 } & 1.1003994313 \\ \text { y37 } & 109.970795975 \\ \text { z37 } & 247.153167703 \\ \text { x38 } & 1.09821275741 \\ \text { y38 } & 110.214183959 \\ \text { z38 } & 128.078515233 \\ \text { x39 } & 1.41169347637 \\ \text { y39 } & 119.480240646 \\ \text { z39 } & 97.8213122899 \\ \text { x40 } & 1.41350538347 \\ \text { y40 } & 118.646828766 \\ \text { z40 } & 267.322897986 \\ \text { x41 } & 1.55388992129 \\ \text { y41 } & 111.726181828 \\ \text { z41 } & 187.822011177 \\ \text { x42 } & 1.1004847965 \\ \text { y42 } & 110.421531866 \\ \text { z42 } & 308.958506151 \\ \text { x43 } & 1.09995477776 \\ \text { y43 } & 109.253940216 \\ \text { z43 } & 65.8811572914 \\ \text { x44 } & 1.55251802436 \\ \text { y44 } & 109.394093935 \\ \text { z44 } & 199.926525549 \\ \text { x45 } & 1.09737982554 \\ \text { y45 } & 112.554719566 \\ \text { z45 } & 321.281303381 \\ \text { x46 } & 1.10168128162 \\ \text { y46 } & 109.609896493 \\ \text { z46 } & 79.4686133997 \\ \text { x47 } & 1.5482723911 \\ \text { y47 } & 110.075239305 \\ \text { z47 } & 195.47859718 \\ \text { x48 } & 1.09972816799 \\ \text { y48 } & 112.106564053 \\ \text { z48 } & 318.883256317 \\ \text { x49 } & 1.10254287112 \\ \text { y49 } & 108.648236359 \\ \text { z49 } & 75.0855892103\end{array}$




$$
\begin{array}{ll}
\text { x50 } & 1.55030110157 \\
\text { y50 } & 111.511555828 \\
\text { z50 } & 198.703156065 \\
\text { x51 } & 1.10189020575 \\
\text { y51 } & 110.527259845 \\
\text { z51 } & 321.105362782 \\
\text { x52 } & 1.10214778125 \\
\text { y52 } & 109.147642115 \\
\text { z52 } & 77.8526416404 \\
\text { x53 } & 1.54810113781 \\
\text { y53 } & 109.46949818 \\
\text { z53 } & 200.062772401 \\
\text { x54 } & 1.09808839583 \\
\text { y54 } & 110.396533136 \\
\text { z54 } & 320.798535187 \\
\text { x55 } & 1.10153456789 \\
\text { y55 } & 110.203805783 \\
\text { z55 } & 78.8514134939 \\
\text { x56 } & 1.55078197029 \\
\text { y56 } & 111.572306073 \\
\text { z56 } & 193.97057207 \\
\text { x57 } & 1.09871579947 \\
\text { y57 } & 110.618699977 \\
\text { z57 } & 315.622890755 \\
\text { x58 } & 1.10090292349 \\
\text { y58 } & 109.316191077 \\
\text { z58 } & 72.4610485371 \\
\text { x59 } & 1.39890018192 \\
\text { y59 } & 119.864648251 \\
\text { z59 } & 359.264366479 \\
\text { x60 } & 1.09130119823 \\
\text { y60 } & 119.903552388 \\
\text { z60 } & 179.463883674 \\
\text { x61 } & 1.09184253555 \\
\text { y61 } & 119.530516859 \\
\text { z61 } & 179.742636096 \\
x 62 & 1.39870200389 \\
\text { y62 } & 118.251651134 \\
\text { z62 } & 188.509210766 \\
x 63 & 1.50800620085 \\
\text { y63 } & 121.060776311 \\
\text { z63 } & 8.20332508622 \\
\text { x64 } & 1.40294254447 \\
\text { y64 } & 117.982203302 \\
\text { z64 } & 171.739689666 \\
\text { x65 } & 1.5080741055 \\
\text { y65 } & 121.705016199 \\
\text { z65 } & 352.308411134 \\
\text { x66 } & 1.40262160373 \\
\text { y66 } & 118.178599682 \\
\text { z66 } & 172.798998002 \\
\text { x67 } & 1.50637351756 \\
\text { y67 } & 122.059524562 \\
\text { z67 } & 354.460142879 \\
\text { x68 } & 1.40062285115 \\
\text { y68 } & 118.259897623 \\
\text { z68 } & 187.371977082
\end{array}
$$




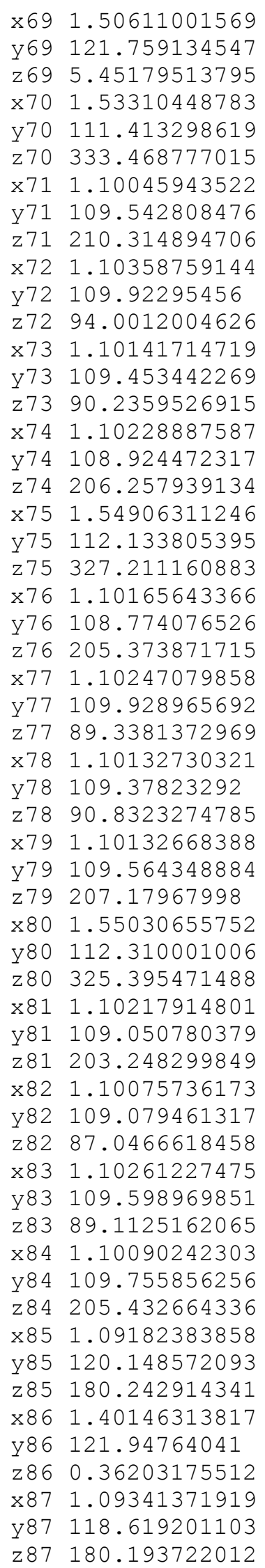




$$
\begin{array}{ll}
\text { x88 } & 1.10021401168 \\
\text { y88 } & 111.397517175 \\
\text { z88 } & 54.3870255528 \\
\text { x89 } & 1.09707980522 \\
\text { y89 } & 110.521548301 \\
\text { z89 } & 175.336382285 \\
\text { x90 } & 1.10199393296 \\
\text { y90 } & 111.569006507 \\
\text { z90 } & 294.936416377 \\
\text { x91 } & 1.0935469799 \\
\text { y91 } & 118.522953541 \\
\text { z91 } & 179.742619668 \\
\text { x92 } & 1.10075475474 \\
\text { y92 } & 111.754924223 \\
\text { z92 } & 307.64288561 \\
\text { x93 } & 1.10189727701 \\
\text { y93 } & 111.494424239 \\
\text { z93 } & 67.5498005223 \\
\text { x94 } & 1.09751145222 \\
\text { y94 } & 110.415085514 \\
\text { z94 } & 186.628296552 \\
\text { x95 } & 1.39803615138 \\
\text { y95 } & 122.237099475 \\
\text { z95 } & 358.075899754 \\
\text { x96 } & 1.09324554113 \\
\text { y96 } & 118.374209604 \\
\text { z96 } & 179.291760138 \\
\text { x97 } & 1.10019931179 \\
\text { y97 } & 111.219364537 \\
\text { z97 } & 303.708310677 \\
\text { x98 } & 1.10144188 \\
\text { y98 } & 111.36531809 \\
\text { z98 } & 63.8772591404 \\
\text { x99 } & 1.09728528248 \\
\text { y99 } & 110.357065277 \\
\text { z99 } & 183.068225553 \\
\text { x100 } & 1.09331403255 \\
\text { y100 } & 118.402165456 \\
\text { z100 } & 180.247186562 \\
\text { x101 } & 1.10126078907 \\
\text { y101 } & 111.383838487 \\
\text { z101 } & 301.811007705 \\
\text { x102 } & 1.10136660461 \\
\text { y102 } & 111.382020936 \\
\text { z102 } & 61.3860991538 \\
\text { x103 } & 1.09729292192 \\
\text { y103 } & 110.396263895 \\
\text { z103 } & 182.275152595 \\
\text { x104 } & 1.101975507 \\
\text { y104 } & 108.967549783 \\
\text { z104 } & 302.492719384 \\
\text { x105 } & 1.10102570146 \\
\text { y105 } & 110.977837706 \\
\text { z105 } & 185.713071866 \\
\text { x106 } & 1.10121137006 \\
\text { y106 } & 110.248217261 \\
\text { z106 } & 207.144876973
\end{array}
$$




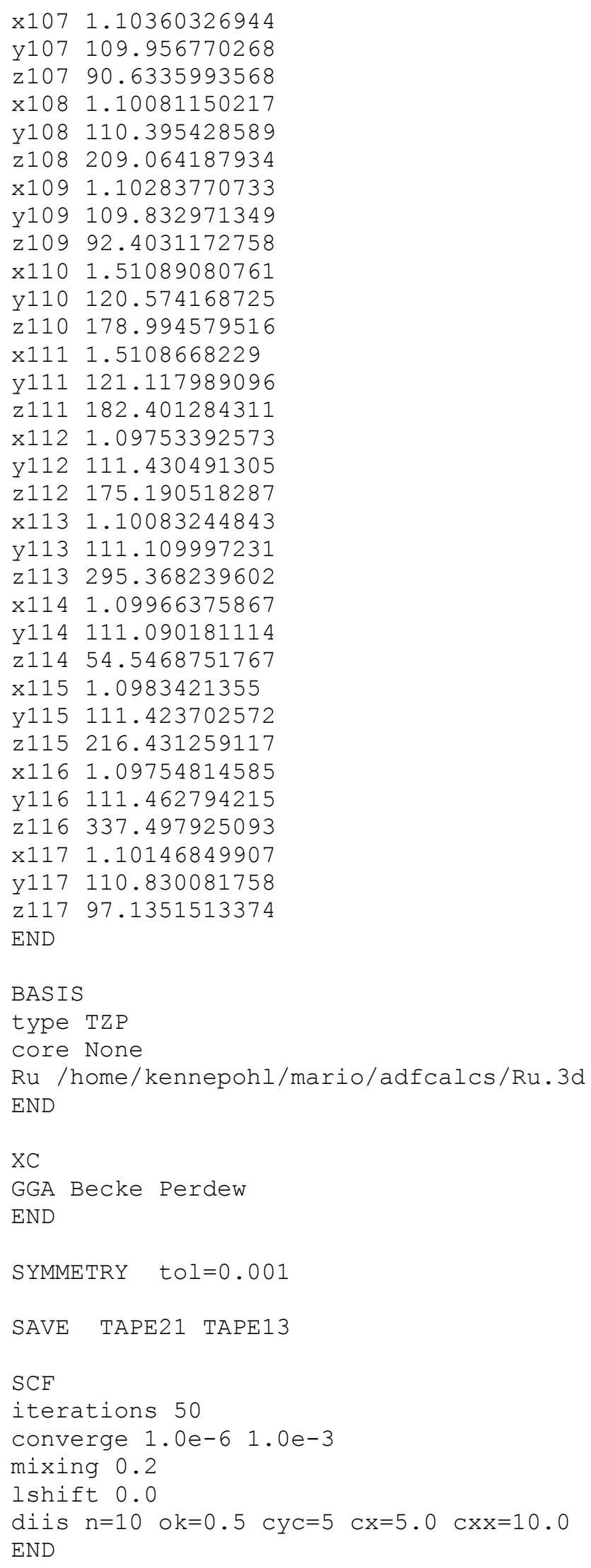


INTEGRATION $3.0 \quad 6.0 \quad 6.0$

A1FIT 10.0

occupations Keeporbitals=100

VECTORLENGTH 256

MAXMEMORYUSAGE 756

eor 
S17: ADF input file for ground state calculation on first generation Piers catalyst (2a). Input geometry was previously optimized (Up to an integration level of 6.0) using the same density functional (Becke and Perdew) and basis set parameters (TZP). A ruthenium $3 \mathrm{~d}$ core was used in this calculation. Complete output for this calculation will be provided upon request (Pierre@chem.ubc.ca.)

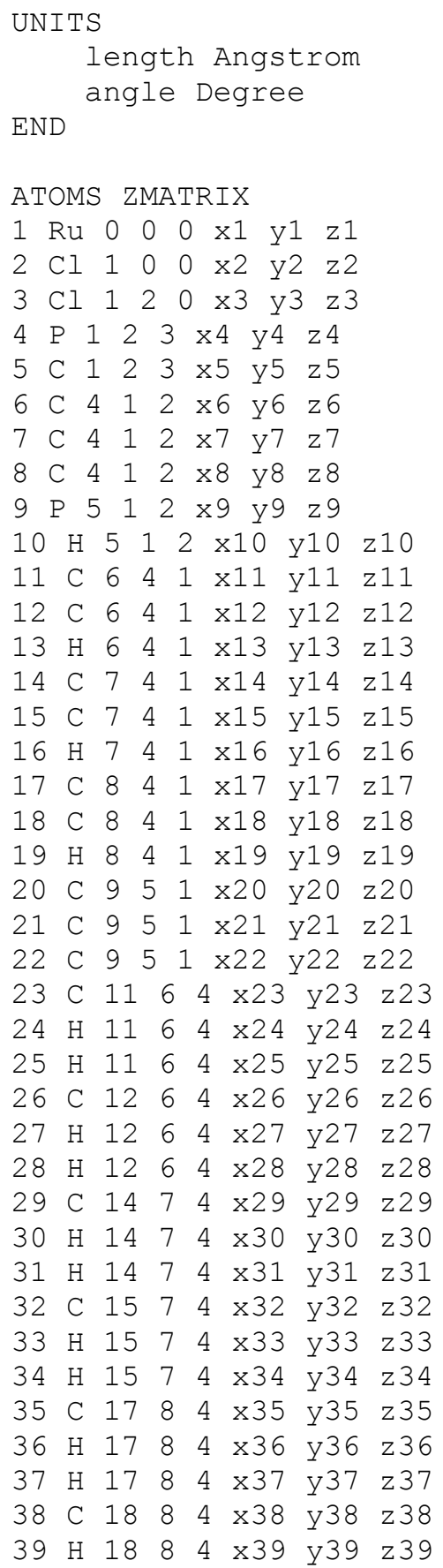


$\begin{array}{lllllllll}40 & \mathrm{H} & 18 & 8 & 4 & \mathrm{x} 40 & \text { y40 z40 }\end{array}$

$\begin{array}{llllllllll}41 & \mathrm{C} & 20 & 9 & 5 & \mathrm{x} 41 & \mathrm{y} 41 & \mathrm{z} 41\end{array}$

$\begin{array}{llllllll}42 & \mathrm{C} & 20 & 9 & 5 & \mathrm{x} 42 & \mathrm{y} 42 & \mathrm{z} 42\end{array}$

$\begin{array}{lllllllll}43 & \mathrm{H} & 20 & 9 & 5 & \mathrm{x} 43 & \mathrm{y} 43 & \mathrm{z} 43\end{array}$

$\begin{array}{lllllllll}44 & \mathrm{C} & 21 & 9 & 5 & \mathrm{x} 44 & \mathrm{y} 44 & \mathrm{z} 44\end{array}$

$\begin{array}{lllllllll}45 & \mathrm{C} & 21 & 9 & 5 & \mathrm{x} 45 & \mathrm{y} 45 & \mathrm{z} 45\end{array}$

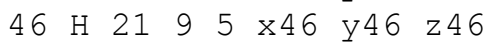

$\begin{array}{lllllllll}47 & \mathrm{C} & 22 & 9 & 5 & \mathrm{x} 47 & \mathrm{y} 47 & \mathrm{z} 47\end{array}$

$\begin{array}{lllllllll}48 & \mathrm{C} & 22 & 9 & 5 & \mathrm{x} 48 & \text { y48 } & \mathrm{z} 48\end{array}$

49 H $22 \quad 9 \quad 5 \quad x 49$ y49 z49

$\begin{array}{llllllll}50 & \mathrm{C} & 23 & 11 & 6 & \mathrm{x} 50 & \text { y50 } & \mathrm{z} 50\end{array}$

$\begin{array}{lllllllll}51 & \mathrm{H} & 23 & 11 & 6 & \mathrm{x} 51 & \mathrm{y} 51 & \mathrm{z} 51\end{array}$

$\begin{array}{llllllll}52 & \mathrm{H} & 23 & 11 & 6 & \mathrm{x} 52 & \mathrm{y} 52 & \mathrm{z} 52\end{array}$

$\begin{array}{lllllll}53 & \mathrm{H} & 26 & 12 & 6 & x 53 & \text { y53 z53 }\end{array}$

$\begin{array}{lllllllll}54 & \mathrm{H} & 26 & 12 & 6 & \mathrm{x} 54 & \mathrm{y} 54 & \mathrm{z} 54\end{array}$

$\begin{array}{lllllllll}55 & \mathrm{C} & 29 & 14 & 7 & \mathrm{x} 55 & \text { y55 z55 }\end{array}$

$\begin{array}{llllllll}56 & \text { H } & 29 & 14 & 7 & \text { x } 56 & \text { y } 56 & \text { z } 56\end{array}$

$\begin{array}{llllllll}57 & \mathrm{H} & 29 & 14 & 7 & \mathrm{x} 57 & \mathrm{y} 57 & \mathrm{z} 57\end{array}$

$\begin{array}{lllllllll}58 & \mathrm{H} & 32 & 15 & 7 & \times 58 & \text { y58 } & \mathrm{z} 58\end{array}$

$\begin{array}{llllllll}59 & \mathrm{H} & 32 & 15 & 7 & \times 59 & \text { y59 z59 }\end{array}$

$\begin{array}{llllllll}60 & \mathrm{C} & 35 & 17 & 8 & \mathrm{x} 60 & \text { y } 60 & \mathrm{z} 60\end{array}$

$\begin{array}{lllllllll}61 & \mathrm{H} & 35 & 17 & 8 & \mathrm{x} 61 & \mathrm{y} 61 & \mathrm{z} 61\end{array}$

$\begin{array}{lllllllll}62 & \mathrm{H} & 35 & 17 & 8 & \mathrm{x} 62 & \mathrm{y} 62 & \mathrm{z} 62\end{array}$

$\begin{array}{llllllll}63 & \mathrm{H} & 38 & 18 & 8 & \mathrm{x} 63 & \mathrm{y} 63 & \mathrm{z} 63\end{array}$

$\begin{array}{lllllllll}64 & \mathrm{H} & 38 & 18 & 8 & \mathrm{x} 64 & \mathrm{y} 64 & \mathrm{z} 64\end{array}$

65 C $4120 \quad 9$ x65 y65 z65

$\begin{array}{llllllll}66 & \mathrm{H} & 41 & 20 & 9 & \mathrm{x} 66 & \text { y } 66 & \text { z } 66\end{array}$

$\begin{array}{llllllll}67 & \mathrm{H} & 41 & 20 & 9 & \mathrm{x} 67 & \text { y } 67 & \mathrm{z} 67\end{array}$

68 C $42209 \times 68$ y68 z68

$69 \mathrm{H} 42 \quad 20 \quad 9$ x69 y69 z69

$\begin{array}{lllllllll}70 & \mathrm{H} & 42 & 20 & 9 & \mathrm{x} 70 & \text { y70 } & \text { z70 }\end{array}$

$\begin{array}{lllllllll}71 & \mathrm{C} & 44 & 21 & 9 & \mathrm{x} 71 & \mathrm{y} 71 & \mathrm{z} 71\end{array}$

$\begin{array}{llllllll}72 & \mathrm{H} & 44 & 21 & 9 & \mathrm{x} 72 & y 72 & \mathrm{z} 72\end{array}$

$\begin{array}{llllllll}73 & \mathrm{H} & 44 & 21 & 9 & \mathrm{x} 73 & \text { y73 } & \mathrm{z} 73\end{array}$

$\begin{array}{llllllll}74 & \mathrm{C} & 45 & 21 & 9 & \mathrm{x} 74 & \mathrm{y} 74 & \mathrm{z} 74\end{array}$

$\begin{array}{llllllll}75 & \mathrm{H} & 45 & 21 & 9 & x 75 & \text { y75 } & \text { z75 }\end{array}$

$\begin{array}{lllllllll}76 & \mathrm{H} & 45 & 21 & 9 & \mathrm{x} 76 & \text { y76 } & \mathrm{z} 76\end{array}$

$\begin{array}{lllllllll}77 & \mathrm{C} & 47 & 22 & 9 & \mathrm{x} 77 & \mathrm{y} 77 & \mathrm{z} 77\end{array}$

$\begin{array}{llllllll}78 & \mathrm{H} & 47 & 22 & 9 & \mathrm{x} 78 & \mathrm{y} 78 & \mathrm{z} 78\end{array}$

$\begin{array}{llllllll}79 & \mathrm{H} & 47 & 22 & 9 & x 79 & \text { y79 } & \text { z } 79\end{array}$

80 C $48 \quad 22 \quad 9 \quad x 80$ y80 z80

$\begin{array}{lllllllll}81 & \mathrm{H} & 48 & 22 & 9 & \mathrm{x} 81 & \mathrm{y} 81 & \mathrm{z} 81\end{array}$

$82 \mathrm{H} \quad 4822 \quad 9 \quad x 82$ y82 z82

$83 \mathrm{H} 50 \quad 23 \quad 11$ x83 y83 z83

$\begin{array}{lllllllll}84 & \mathrm{H} & 50 & 23 & 11 & \mathrm{x} 84 & \text { y84 } & \mathrm{z} 84\end{array}$

$\begin{array}{llllllll}85 & \mathrm{H} & 55 & 29 & 14 & x 85 & \text { y85 } & \text { z } 85\end{array}$

$\begin{array}{lllllllll}86 & \mathrm{H} & 55 & 29 & 14 & \mathrm{x} 86 & \text { y8 } 86 & \mathrm{z} 86\end{array}$

$\begin{array}{lllllllll}87 & \mathrm{H} & 60 & 35 & 17 & \mathrm{x} 87 & \mathrm{y} 87 & \mathrm{z} 87\end{array}$

$\begin{array}{llllllllll}88 & \mathrm{H} & 60 & 35 & 17 & \mathrm{x} 88 & \mathrm{y} 88 & \mathrm{z} 88\end{array}$

89 C $65 \quad 4120 \times 89$ y89 z89

$90 \mathrm{H} \quad 65 \quad 4120 \times 90 \quad$ y90 z90

$\begin{array}{lllllllll}91 & \mathrm{H} & 65 & 41 & 20 & \times 91 & \text { y91 } & \text { z } 91\end{array}$

$92 \mathrm{H} \quad 68 \quad 42 \quad 20 \quad x 92 \quad$ y92 z92

$93 \mathrm{H} \quad 68 \quad 42 \quad 20 \quad x 93$ y93 z93

$\begin{array}{lllllllll}94 & \mathrm{C} & 71 & 44 & 21 & \times 94 & \text { y94 } & \text { z94 }\end{array}$

$\begin{array}{llllllll}95 & \mathrm{H} & 71 & 44 & 21 & \times 95 & \text { y95 z95 }\end{array}$

$\begin{array}{lllllllll}96 & \mathrm{H} & 71 & 44 & 21 & \times 96 & \text { y96 } & \text { z } 96\end{array}$ 
$\begin{array}{lllllllll}97 & \mathrm{H} & 74 & 45 & 21 & \times 97 & \text { y97 } & \text { z } 97\end{array}$

$\begin{array}{lllllllll}98 & \mathrm{H} & 74 & 45 & 21 & \times 98 & \text { y98 } & \text { z } 98\end{array}$

99 C $77 \quad 47 \quad 22 \quad x 99$ y99 z99

$100 \mathrm{H} \quad 77 \quad 47 \quad 22 \times 100$ y100 z100

$101 \mathrm{H} \quad 77 \quad 4722 \times 101$ y101 z101

$102 \mathrm{H} \quad 80 \quad 48 \quad 22 \times 102$ y102 z102

$103 \mathrm{H} \quad 80 \quad 48 \quad 22 \times 103$ y103 z103

$104 \mathrm{H} \quad 8965 \quad 41 \times 104 \quad$ y104 z104

$105 \mathrm{H} \quad 89 \quad 65 \quad 41$ x105 y105 z105

$106 \mathrm{H} \quad 9471 \quad 44 \times 106$ y106 z106

$\begin{array}{lllllllll}107 & \mathrm{H} & 94 & 71 & 44 & \mathrm{x} 107 & \text { y107 } & \mathrm{z} 107\end{array}$

$108 \mathrm{H} \quad 99 \quad 77 \quad 47 \times 108$ y108 z108

$109 \mathrm{H} 997747 \times 109$ y109 z109

END
GEOVAR
$\mathrm{x} 10.0$
y1 0.0
z1 0.0
$x 22.32282527071$
y2 0.0
z2 0.0
$\times 32.32380389649$
y3 142.516415862
z 30.0
$\times 42.31449835053$
y4 93.1847176796
z4 103.16974467
$\times 51.84909192291$
y5 108.252424855
z5 203.38269309
x61.88997942854
y6 112.312971083
z6 173.761818391
x7 1.88569126058
y7 111.013926457
z7 289.528882111
$x 81.88322187061$
y8 113.216398468
z8 44.9923638526
×91.81889599389
y9 126.05318922
z9 84.575435953
$\times 101.10160709752$
y10 124.095097455
z10 266.300975927
x11 1.54399690612
y11 113.447269608
z11 284.781550764
x12 1.54865095437
y12 116.132774407
z12 155.681659074
x131.10498175106
y13 101.030299517
z13 39.5775415772
x14 1.54388486641
y14 113.44097265 


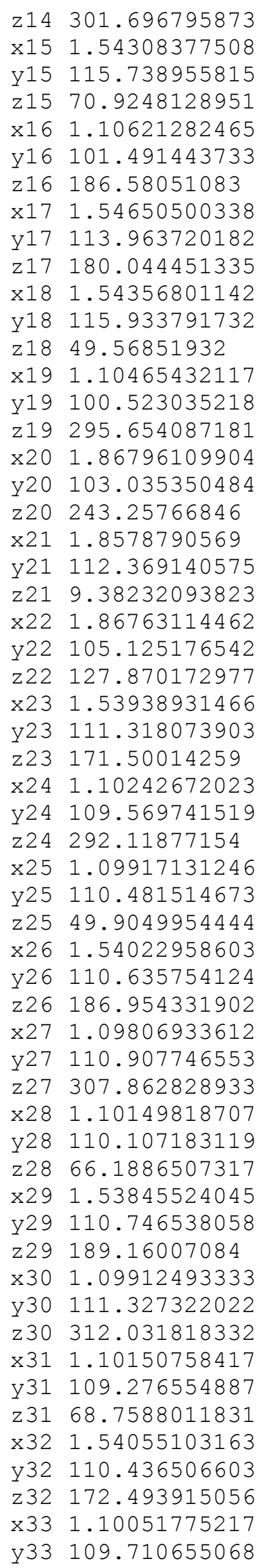




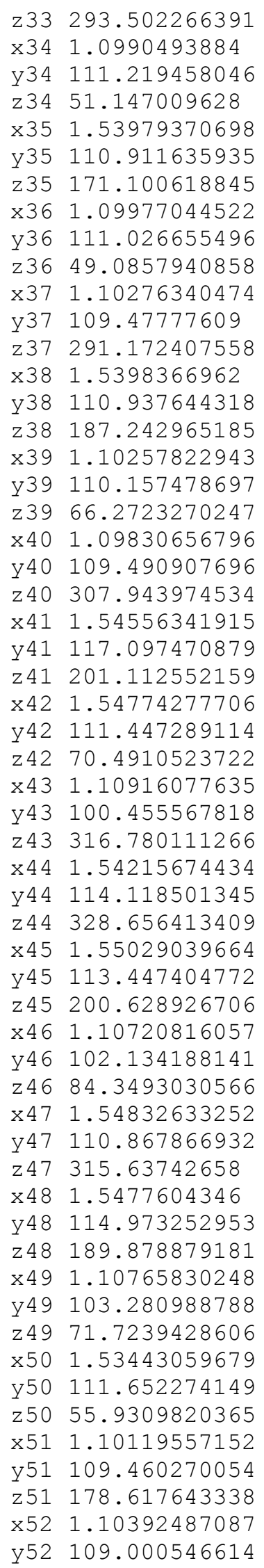


z52 294.990536699

x531.10064144917

y53 109.099540665

z53 181.01469442

$\times 541.10408034995$

y54 109.043279564

z54 65.075701135

x55 1.53517654288

y55 111.578588595

z55 303.824815257

$\times 561.1048604698$

y56 109.342076539

z56 65.0787550373

$\times 571.1007051267$

y57 109.289020004

z57 181.245680534

x581.10080258342

y58 109.122514669

z58 178.440630871

x591.10477653222

y59 109.21854907

z59 294.401343861

×601.53407915652

y60 111.630360646

z60 56.0956993378

x61 1.10397032781

y61 109.199563184

z61 294.865257743

x62 1.10073498202

y62 109.226485953

z62 178.714651026

х631.10125504249

y63 109.439821804

z63 182.006466896

x64 1.10387419607

y64 108.774725878

z64 66.000326997

×651.5409687226

y65 110.190408109

z65 174.428582469

x66 1.09936399686

y66 110.74181332

z66 53.476253158

x671.10169631691

y67 109.931749773

z67 295.028727811

x68 1.53864257549

y68 110.614191009

z68 188.741503592

x691.09966631011

y69 110.733055555

z69 311.429820199

x70 1.10245702741

y70 109.254987055

z70 68.9042945488

x71 1.53774179916

y71 111.077762622 


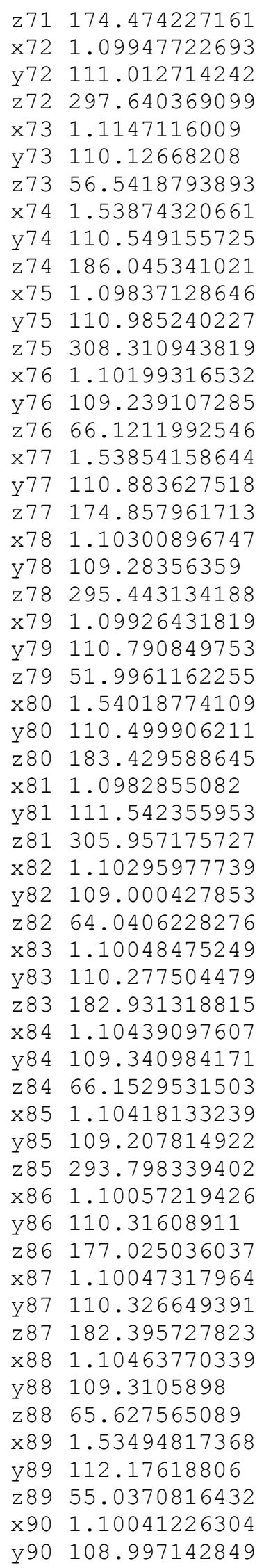




$$
\begin{array}{ll}
\text { z90 } & 177.947307692 \\
\text { x91 } & 1.10432683065 \\
\text { y91 } & 108.972367373 \\
\text { z91 } & 293.719903535 \\
\text { x92 } & 1.10018952645 \\
\text { y92 } & 109.091856083 \\
\text { z92 } & 182.136924199 \\
\text { x93 } & 1.10413033337 \\
\text { y93 } & 109.16835288 \\
\text { z93 } & 66.1807687971 \\
\text { x94 } & 1.53596204853 \\
\text { y94 } & 111.586765509 \\
\text { z94 } & 55.549879872 \\
\text { x95 } & 1.09957172684 \\
\text { y95 } & 109.001329066 \\
\text { z95 } & 178.146334233 \\
\text { x96 } & 1.10308501898 \\
\text { y96 } & 109.02378246 \\
\text { z96 } & 294.275333221 \\
\text { x97 } & 1.10394515638 \\
\text { y97 } & 109.246707777 \\
\text { z97 } & 65.2855949992 \\
\text { x98 } & 1.10027848985 \\
\text { y98 } & 109.071947655 \\
\text { z98 } & 181.365898879 \\
\text { x99 } & 1.53513295132 \\
\text { y99 } & 111.980302571 \\
\text { z99 } & 55.4172857031 \\
\text { x100 } & 1.10024206734 \\
\text { y100 } & 109.074411265 \\
\text { z100 } & 178.064183473 \\
\text { x101 } & 1.1042951285 \\
\text { y101 } & 109.097705534 \\
\text { z101 } & 294.061528467 \\
\text { x102 } & 1.10421328038 \\
\text { y102 } & 109.172099856 \\
\text { z102 } & 64.8541599676 \\
\text { x103 } & 1.10045042717 \\
\text { y103 } & 109.028961982 \\
\text { z103 } & 180.821010371 \\
\text { x104 } & 1.10415137292 \\
\text { y104 } & 109.405251021 \\
\text { z104 } & 65.5799557867 \\
\text { x105 } & 1.10016298011 \\
\text { y105 } & 110.050225104 \\
\text { z105 } & 182.187730917 \\
\text { x106 } & 1.10003176179 \\
\text { y106 } & 109.870395566 \\
\text { z106 } & 182.747046741 \\
\text { x107 } & 1.10390356243 \\
\text { y107 } & 109.487505731 \\
\text { z107 } & 66.0882229557 \\
\text { x108 } & 1.10013310316 \\
\text { y108 } & 110.223171853 \\
\text { z108 } & 183.379323815 \\
\text { x109 } & 1.10409866969 \\
\text { y109 } & 109.333129657
\end{array}
$$




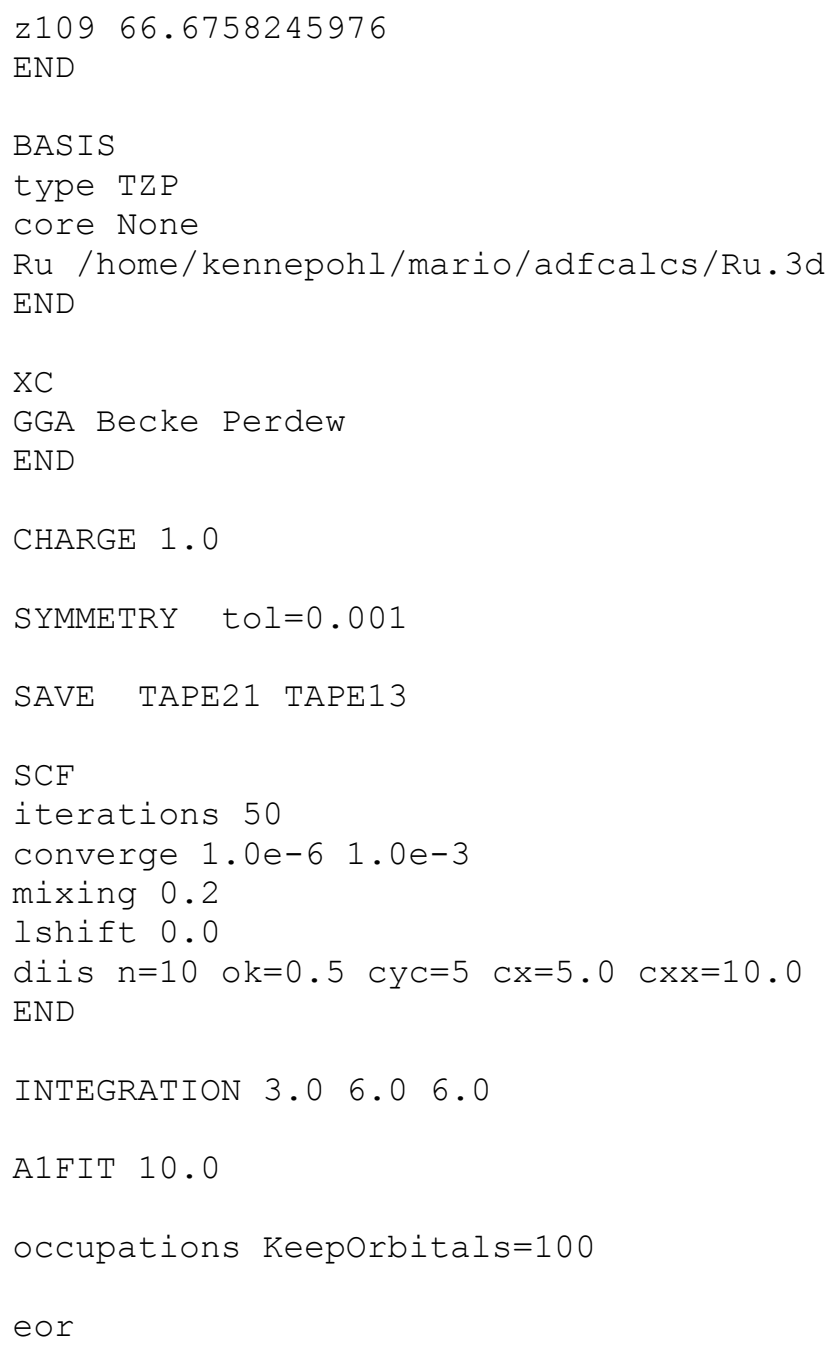


S18: ADF input file for ground state calculation on second generation Piers catalyst (2b). Input geometry was previously optimized (Up to an integration level of 6.0) using the same density functional (Becke and Perdew) and basis set parameters (TZP). A ruthenium 3d core was used in this calculation. Complete output for this calculation will be provided upon request (Pierre@chem.ubc.ca.)

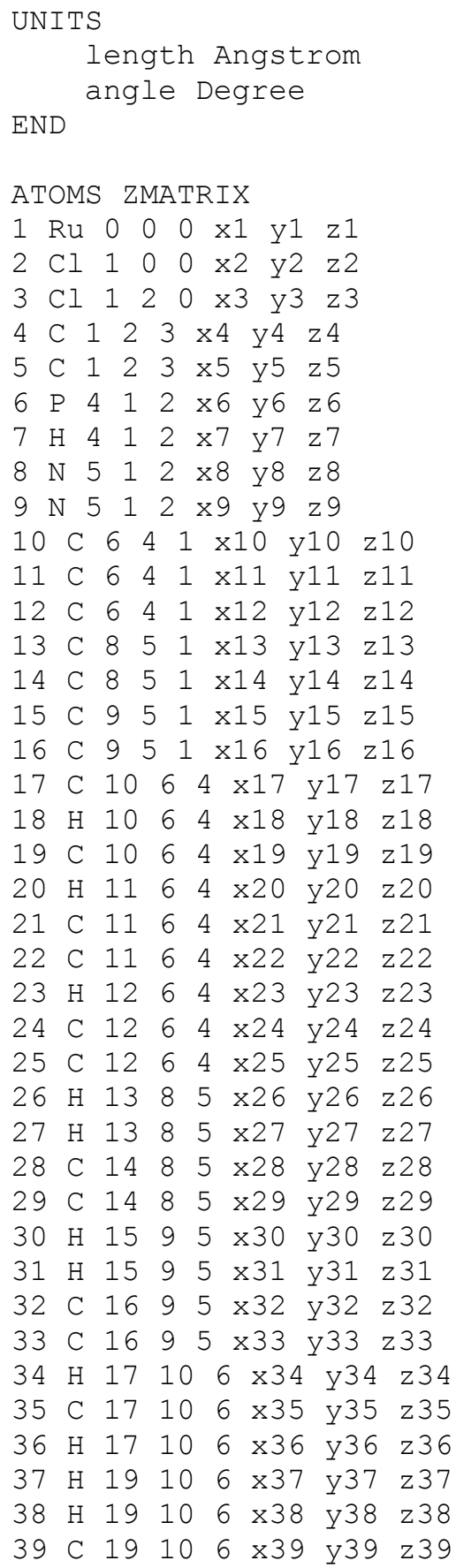


$\begin{array}{lllllllll}40 & \mathrm{H} & 21 & 11 & 6 & \mathrm{x} 40 & \mathrm{y} 40 & \mathrm{z} 40\end{array}$

$\begin{array}{lllllllll}41 & \mathrm{H} & 21 & 11 & 6 & \mathrm{x} 41 & \mathrm{y} 41 & \mathrm{z} 41\end{array}$

$\begin{array}{lllllllll}42 & C & 21 & 11 & 6 & x 42 & y 42 & z 42\end{array}$

$\begin{array}{lllllllll}43 & \mathrm{C} & 22 & 11 & 6 & \mathrm{x} 43 & \mathrm{y} 43 & \mathrm{z} 43\end{array}$

$\begin{array}{lllllllll}44 & \mathrm{H} & 22 & 11 & 6 & \mathrm{x} 44 & \mathrm{y} 44 & \mathrm{z} 44\end{array}$

$\begin{array}{llllllll}45 & \mathrm{H} & 22 & 11 & 6 & \mathrm{x} 45 & \mathrm{y} 45 & \mathrm{z} 45\end{array}$

$\begin{array}{lllllllll}46 & \mathrm{H} & 24 & 12 & 6 & \mathrm{x} 46 & \mathrm{y} 46 & \mathrm{z} 46\end{array}$

$\begin{array}{llllllll}47 & \mathrm{H} & 24 & 12 & 6 & \mathrm{x} 47 & \mathrm{y} 47 & \mathrm{z} 47\end{array}$

$\begin{array}{lllllllll}48 & \mathrm{C} & 24 & 12 & 6 & \mathrm{x} 48 & \mathrm{y} 48 & \mathrm{z} 48\end{array}$

$\begin{array}{llllllll}49 & \mathrm{C} & 25 & 12 & 6 & \mathrm{x} 49 & \text { y49 } & \mathrm{z} 49\end{array}$

$\begin{array}{lllllllll}50 & \mathrm{H} & 25 & 12 & 6 & \times 50 & \text { y50 } & \mathrm{z} 50\end{array}$

$\begin{array}{lllllllll}51 & \mathrm{H} & 25 & 12 & 6 & \mathrm{x} 51 & \mathrm{y} 51 & \mathrm{z} 51\end{array}$

$\begin{array}{llllllll}52 & \mathrm{C} & 28 & 14 & 8 & \mathrm{x} 52 & \mathrm{y} 52 & \mathrm{z} 52\end{array}$

$\begin{array}{lllllllll}53 & C & 28 & 14 & 8 & x 53 & \text { y53 } & \text { z53 }\end{array}$

$\begin{array}{lllllllll}54 & C & 29 & 14 & 8 & x 54 & y 54 & z 54\end{array}$

$\begin{array}{llllllll}55 & \mathrm{C} & 29 & 14 & 8 & x 55 & \text { y55 z55 }\end{array}$

56 C $32 \quad 16 \quad 9 \quad x 56$ y56 z56

$\begin{array}{llllllll}57 & \mathrm{C} & 32 & 16 & 9 & \mathrm{x} 57 & \mathrm{y} 57 & \mathrm{z} 57\end{array}$

$\begin{array}{llllllll}58 & \mathrm{C} & 33 & 16 & 9 & \times 58 & \mathrm{y} 58 & \mathrm{z} 58\end{array}$

59 C $3316 \quad 9 \times 59$ y59 z59

$\begin{array}{lllllllll}60 & \mathrm{C} & 35 & 17 & 10 & \mathrm{x} 60 & \text { y } 60 & \mathrm{z} 60\end{array}$

$\begin{array}{llllllllll}61 & \mathrm{H} & 35 & 17 & 10 & \mathrm{x} 61 & \mathrm{y} 61 & \mathrm{z} 61\end{array}$

$\begin{array}{lllllllll}62 & \mathrm{H} & 35 & 17 & 10 & \mathrm{x} 62 & \mathrm{y} 62 & \mathrm{z} 62\end{array}$

$\begin{array}{lllllllll}63 & \mathrm{H} & 39 & 19 & 10 & \mathrm{x} 63 & \text { y63 } & \text { z } 63\end{array}$

$\begin{array}{llllllll}64 & \mathrm{H} & 39 & 19 & 10 & \mathrm{x} 64 & \text { y64 } & \mathrm{z} 64\end{array}$

$\begin{array}{llllllll}65 & \mathrm{H} & 42 & 21 & 11 & \mathrm{x} 65 & \text { y } 65 & \mathrm{z} 65\end{array}$

$\begin{array}{llllllll}66 & \mathrm{H} & 42 & 21 & 11 & \mathrm{x} 66 & \text { y } 66 & \mathrm{z} 66\end{array}$

$\begin{array}{lllllllll}67 & \mathrm{C} & 42 & 21 & 11 & \mathrm{x} 67 & \mathrm{y} 67 & \mathrm{z} 67\end{array}$

$\begin{array}{lllllllll}68 & \mathrm{H} & 43 & 22 & 11 & \mathrm{x} 68 & \mathrm{y} 68 & \mathrm{z} 68\end{array}$

$\begin{array}{llllllll}69 & \mathrm{H} & 43 & 22 & 11 & x 69 & \text { y69 } & \text { z } 69\end{array}$

$\begin{array}{lllllllll}70 & \mathrm{H} & 48 & 24 & 12 & \mathrm{x} 70 & \mathrm{y} 70 & \mathrm{z} 70\end{array}$

$\begin{array}{lllllllll}71 & \mathrm{H} & 48 & 24 & 12 & x 71 & y 71 & \mathrm{z} 71\end{array}$

$\begin{array}{llllllll}72 & \text { C } & 48 & 24 & 12 & x 72 & y 72 & z 72\end{array}$

$\begin{array}{llllllll}73 & \mathrm{H} & 49 & 25 & 12 & \mathrm{x} 73 & \mathrm{y} 73 & \mathrm{z} 73\end{array}$

$\begin{array}{llllllll}74 & \mathrm{H} & 49 & 25 & 12 & \mathrm{x} 74 & \mathrm{y} 74 & \mathrm{z} 74\end{array}$

$\begin{array}{llllllll}75 & \mathrm{H} & 52 & 28 & 14 & \mathrm{x} 75 & \mathrm{y} 75 & \mathrm{z} 75\end{array}$

$\begin{array}{llllllll}76 & C & 52 & 28 & 14 & x 76 & y 76 & \text { z } 76\end{array}$

$\begin{array}{lllllllll}77 & \mathrm{H} & 53 & 28 & 14 & \mathrm{x} 77 & \mathrm{y} 77 & \mathrm{z} 77\end{array}$

$\begin{array}{llllllll}78 & \mathrm{H} & 53 & 28 & 14 & \mathrm{x} 78 & \mathrm{y} 78 & \mathrm{z} 78\end{array}$

$\begin{array}{lllllllll}79 & \mathrm{H} & 53 & 28 & 14 & \mathrm{x} 79 & \text { y79 } & \text { z } 79\end{array}$

80 H $54 \quad 29 \quad 14 \quad x 80 \quad y 80 \quad z 80$

$\begin{array}{lllllllll}81 & \mathrm{H} & 54 & 29 & 14 & \mathrm{x} 81 & \mathrm{y} 81 & \mathrm{z} 81\end{array}$

$\begin{array}{lllllllll}82 & \mathrm{H} & 54 & 29 & 14 & \mathrm{x} 82 & \text { y82 } & \mathrm{z} 82\end{array}$

$\begin{array}{lllllllll}83 & \mathrm{H} & 55 & 29 & 14 & \mathrm{x} 83 & \mathrm{y} 83 & \mathrm{z} 83\end{array}$

$\begin{array}{lllllllll}84 & \mathrm{H} & 56 & 32 & 16 & \mathrm{x} 84 & \mathrm{y} 84 & \mathrm{z} 84\end{array}$

85 C $56 \quad 32 \quad 16 \quad x 85$ y85 z85

$86 \mathrm{H} \quad 57 \quad 32 \quad 16 \quad x 86 \quad$ y86 z 86

$\begin{array}{lllllllll}87 & \mathrm{H} & 57 & 32 & 16 & \mathrm{x} 87 & \mathrm{y} 87 & \mathrm{z} 87\end{array}$

$\begin{array}{llllllll}88 & \mathrm{H} & 57 & 32 & 16 & \mathrm{x} 88 & \mathrm{y} 88 & \mathrm{z} 88\end{array}$

89 H $58 \quad 33 \quad 16 \quad x 89$ y89 z89

90 H $5933 \quad 16 \times 90$ y90 z90

$\begin{array}{lllllllll}91 & \mathrm{H} & 59 & 33 & 16 & \text { x91 y91 z } 91\end{array}$

92 H $5933 \quad 16$ x92 y92 z92

$\begin{array}{lllllllll}93 & \mathrm{H} & 60 & 35 & 17 & \text { x93 } & \text { y93 } & \text { z93 }\end{array}$

$\begin{array}{lllllllll}94 & \mathrm{H} & 60 & 35 & 17 & \text { x94 } & \text { y94 } & \text { z94 }\end{array}$

$\begin{array}{lllllllll}95 & \mathrm{H} & 67 & 42 & 21 & \times 95 & \text { y95 z95 }\end{array}$

$\begin{array}{lllllllll}96 & \mathrm{H} & 67 & 42 & 21 & \times 96 & \text { y96 } & \text { z } 96\end{array}$ 
$\begin{array}{lllllllll}97 & \mathrm{H} & 72 & 48 & 24 & \times 97 & \text { y97 } & \text { z } 97\end{array}$

$\begin{array}{lllllllll}98 & \mathrm{H} & 72 & 48 & 24 & \mathrm{x} 98 & \text { y98 } & \text { z } 98\end{array}$

99 C $76 \quad 52 \quad 28 \times 99$ y99 z99

$100 \mathrm{C} 855632 \times 100$ y100 z100

$101 \mathrm{H} 997652 \times 101$ y101 z101

$102 \mathrm{H} 997652 \times 102$ y102 z102

$103 \mathrm{H} 997652$ x103 y103 z103

$104 \mathrm{H} \quad 100 \quad 8556 \times 104$ y104 z104

$105 \mathrm{H} \quad 100 \quad 85 \quad 56 \times 105$ y105 z105

$106 \mathrm{H} 1008556$ x106 y106 z106

END
GEOVAR
$\mathrm{x} 10.0$
y1 0.0
z 10.0
$x 22.34138340239$
y2 0.0
z2 0.0
×3 2.3287289605
y3 149.308716679
z3 0.0
×4 1.84749127277
y4 102.956647451
z4 212.851144072
$\times 52.01185505258$
y5 95.1183063931
z5 110.536548937
×6 1.81739821944
y6 126.000618378
z6 76.6417531031
x7 1.10050396114
y7 122.957103291
z7 257.59514331
x8 1.36001621658
y8 131.261846001
z8 94.952677987
x91.3568077458
y9 120.444106698
z9 277.265818256
$\times 101.86248209026$
y10 108.883592779
z10 116.347773856
x11 1.8552119007
y11 111.953841426
z11 352.007597099
x12 1.86170119736
y12 104.537761576
z12 232.240966527
x13 1.48818376238
y13 112.261976568
z13 177.815771711
x14 1.43583033199
y14 130.3005377
z14 3.08007524178
x15 1.48398650954
y15 112.608994688 
z15 173.425986795

x16 1.44590450319

y16 128.687000375

z16 357.680093453

$\times 171.54746475879$

y17 111.571374191

z17 62.9060783646

x18 1.10702903887

y18 104.173732542

z18 179.784181509

x19 1.54691381932

y19 112.001212788

z19 297.392030402

x201.10551808638

y20 103.034379073

z20 277.689285662

x21 1.54287788477

y21 113.691254054

z21 33.5829516299

x22 1.54983709578

y22 112.508736646

z22 161.071178817

x231.10727165696

y23 101.96894934

z23 309.232182591

x241.54529566608

y24 115.044431905

z24 192.338510308

x25 1.54522155604

y25 111.989419996

z25 63.916361288

x261.09772300829

y26 110.021335608

z26 131.721872484

x27 1.10080130373

y27 109.731817431

z27 250.793176491

x28 1.41082375383

y28 119.38434731

z28 91.5566924037

x291.40888726893

y29 118.837593913

z29 266.232691476

x301.09761006972

y30 110.056161121

z30 131.865002874

x311.10102769365

y31 109.528456658

z31 251.102629171

x32 1.41304294697

y32 118.531395328

z32 262.599942522

×331.41316394827

y33 119.761970883

z33 91.1376581663

×341.1028611776

y34 109.298289158 
$\begin{array}{ll}\text { z34 } & 299.508532422 \\ \text { x35 } & 1.53886412503 \\ \text { y35 } & 110.923195569 \\ \text { z35 } & 179.109997703 \\ \text { x36 } & 1.09968837684 \\ \text { y36 } & 110.849310955 \\ \text { z36 } & 57.056290898 \\ \text { x37 } & 1.09929490945 \\ \text { y37 } & 110.61197935 \\ \text { z37 } & 302.176850022 \\ \text { x38 } & 1.10259400855 \\ \text { y38 } & 109.392622532 \\ \text { z38 } & 59.0188763302 \\ \text { x39 } & 1.5387796389 \\ \text { y39 } & 110.957557246 \\ \text { z39 } & 180.443353264 \\ \text { x40 } & 1.11571926005 \\ \text { y40 } & 110.304585778 \\ \text { z40 } & 300.901810522 \\ \text { x41 } & 1.09884195995 \\ \text { y41 } & 111.364130089 \\ \text { z41 } & 59.6654904311 \\ \text { x42 } & 1.53781417596 \\ \text { y42 } & 111.259915664 \\ \text { z42 } & 183.095740857 \\ \text { x43 } & 1.5383659926 \\ \text { y43 } & 110.839759472 \\ \text { z43 } & 176.030930656 \\ \text { x44 } & 1.09949128264 \\ \text { y44 } & 110.740781043 \\ \text { z44 } & 53.710002015 \\ \text { x45 } & 1.10243798125 \\ \text { y45 } & 109.450835508 \\ \text { z45 } & 296.096618856 \\ \text { x46 } & 1.10036864913 \\ \text { y46 } & 111.266589486 \\ \text { z46 } & 52.2270937457 \\ \text { x47 } & 1.10271522604 \\ \text { y47 } & 109.580182547 \\ \text { z47 } & 293.971724509 \\ \text { x48 } & 1.54065778745 \\ \text { y48 } & 110.088767622 \\ \text { z48 } & 174.251809758 \\ \text { x49 } & 1.53917518823 \\ \text { y49 } & 110.220651533 \\ \text { z49 } & 187.311749751 \\ \text { x50 } & 1.10285107 \\ \text { y50 } & 109.612606342 \\ \text { z50 } & 67.507228194 \\ \text { x51 } & 1.09994287559 \\ \text { y51 } & 110.674032448 \\ \text { z51 } & 310.126726428 \\ \text { x52 } & 1.40134883976 \\ \text { y52 } & 118.077077422 \\ \text { z52 } & 175.48124491 \\ \text { x53 } & 1.50913151092 \\ \text { y53 } & 121.504891343\end{array}$ 
z53 354.829375465

$\times 541.50761226377$

y54 121.324855943

z54 4.31439550654

×551.40190519145

y55 118.039666384

z55 184.423390544

$\times 561.39942280035$

y56 118.095310483

z56 182.496606466

$\times 571.50880806557$

y57 122.542009768

z57 358.621884001

$\times 581.40175011282$

y58 117.968675916

z58 177.671286776

x591.50928915509

y59 123.040869054

z59 2.81302020659

x60 1.53488432517

y60 111.778532176

z60 55.455643929

x61 1.10420928942

y61 109.237786053

z61 294.151124994

x621.10096840173

y62 109.101533725

z62 178.219516056

x631.10023894952

y63 108.998996652

z63 182.230541644

x641.1043962727

y64 109.335339679

z64 66.1836979997

x65 1.09947894559

y65 108.953810281

z65 182.319408265

x66 1.10301512824

y66 109.114868452

z66 66.0688102249

$\times 671.53660560814$

y67 111.520186706

z67 304.868766792

$\times 681.10385708768$

y68 109.30267378

z68 294.50195257

x691.10033240679

y69 109.1034646

z69 178.31598911

x70 1.10431006757

y70 108.982180368

z70 294.335558585

x71 1.10050304094

y71 109.262583918

z71 178.337536608

x72 1.53611601699

y72 111.79529277 


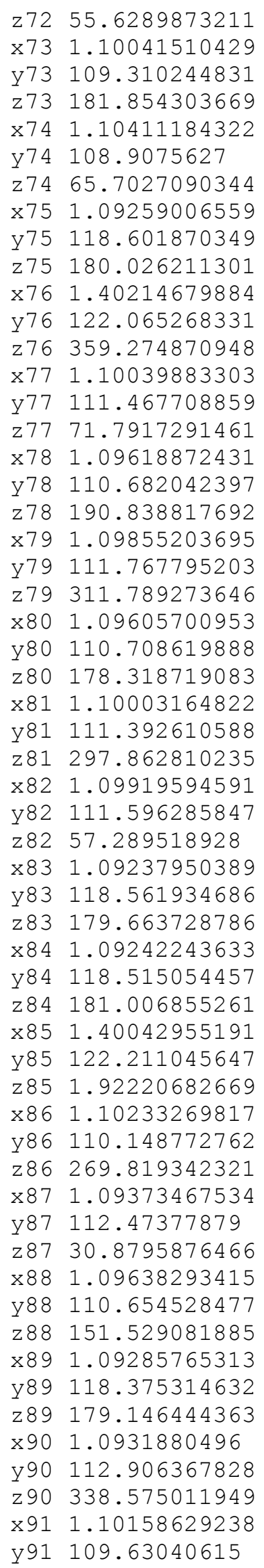




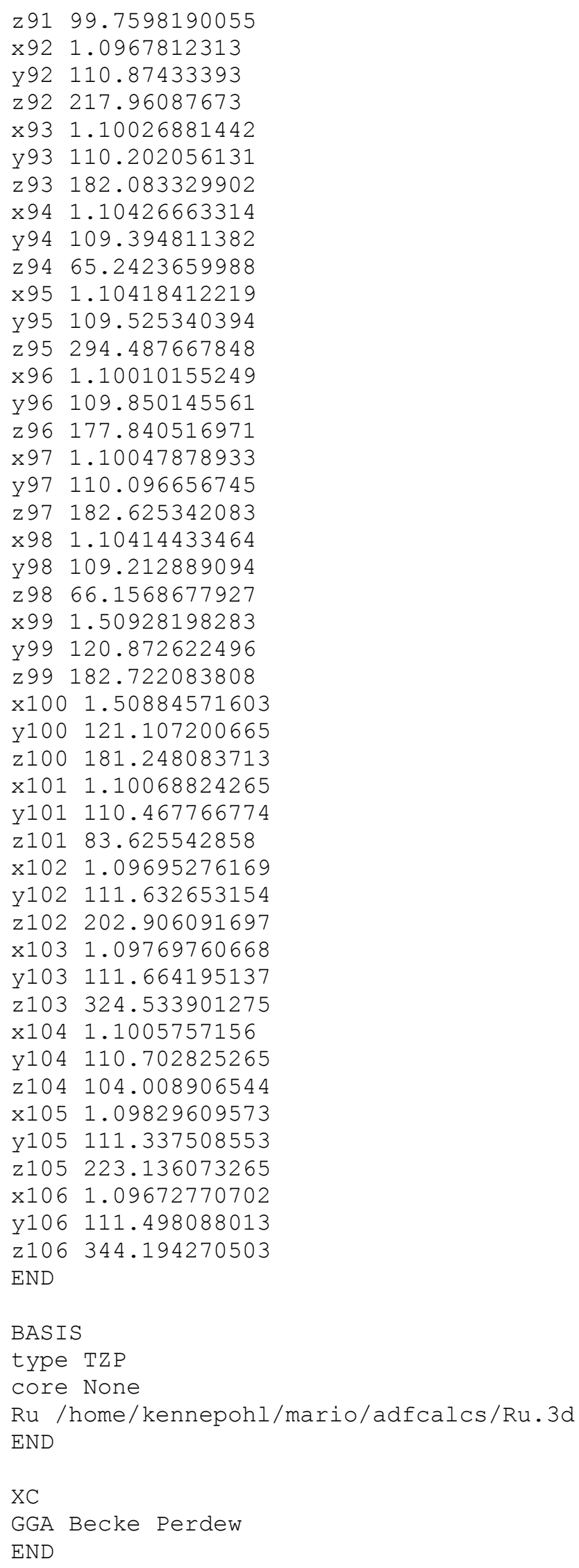


CHARGE 1.0

SYMMETRY tol $=0.001$

SAVE TAPE21 TAPE13

$\mathrm{SCF}$

iterations 50

converge $1.0 e-61.0 e-3$

mixing 0.2

lshift 0.0

diis $\mathrm{n}=10 \quad \mathrm{ok}=0.5 \quad \mathrm{cyc}=5 \quad \mathrm{cx}=5.0 \quad \mathrm{cxx}=10 \cdot 0$

END

INTEGRATION $3.0 \quad 6.0 \quad 6.0$

A1FIT 10.0

Occupations Keeporbitals $=100$

eor 
S19: ADF input file for ground state calculation on compound 3a. Input geometry was previously optimized (Up to an integration level of 4.0) using the same density functional (Becke and Perdew) and basis set parameters (TZP). A ruthenium 3d core was used in this calculation. Complete output for this calculation will be provided upon request (Pierre@chem.ubc.ca.)

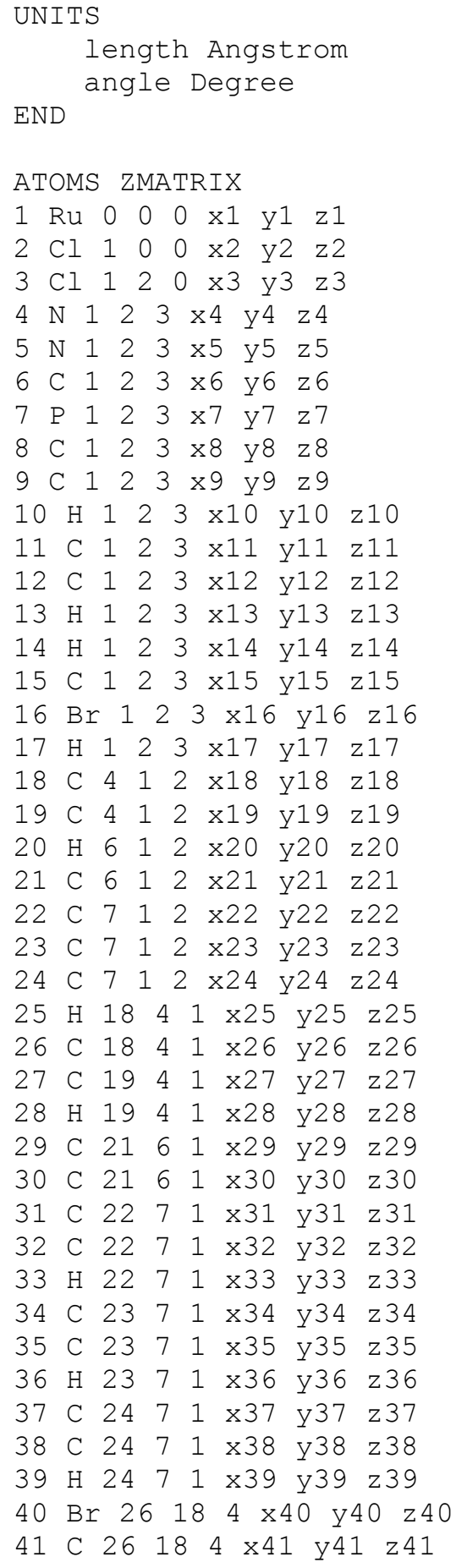


$\begin{array}{llllllll}42 & \mathrm{H} & 27 & 19 & 4 & \mathrm{x} 42 & \text { y42 } & \mathrm{z} 42\end{array}$

$\begin{array}{llllllll}43 & \mathrm{H} & 29 & 21 & 6 & \mathrm{x} 43 & \text { y43 } & \mathrm{z} 43\end{array}$

$\begin{array}{lllllllll}44 & \text { C } & 29 & 21 & 6 & x 44 & y 44 & \text { z } 44\end{array}$

$\begin{array}{lllllllll}45 & \mathrm{C} & 30 & 21 & 6 & \mathrm{x} 45 & \mathrm{y} 45 & \mathrm{z} 45\end{array}$

$\begin{array}{lllllllll}46 & \mathrm{H} & 30 & 21 & 6 & \mathrm{x} 46 & \text { y } 46 & \mathrm{z} 46\end{array}$

$\begin{array}{lllllllll}47 & \mathrm{C} & 31 & 22 & 7 & \mathrm{x} 47 & \mathrm{y} 47 & \mathrm{z} 47\end{array}$

$\begin{array}{lllllllll}48 & \mathrm{H} & 31 & 22 & 7 & \mathrm{x} 48 & \mathrm{y} 48 & \mathrm{z} 48\end{array}$

$\begin{array}{lllllllll}49 & \mathrm{H} & 31 & 22 & 7 & \mathrm{x} 49 & \mathrm{y} 49 & \mathrm{z} 49\end{array}$

$\begin{array}{lllllllll}50 & \mathrm{C} & 32 & 22 & 7 & \mathrm{x} 50 & \text { y50 } & \mathrm{z} 50\end{array}$

$\begin{array}{lllllllll}51 & \mathrm{H} & 32 & 22 & 7 & \mathrm{x} 51 & \text { y51 } & \mathrm{z} 51\end{array}$

$\begin{array}{llllllll}52 & \mathrm{H} & 32 & 22 & 7 & \mathrm{x} 52 & \mathrm{y} 52 & \mathrm{z} 52\end{array}$

53 C $3423 \quad 7 \quad x 53$ y53 z53

$\begin{array}{lllllllll}54 & \mathrm{H} & 34 & 23 & 7 & \mathrm{x} 54 & \mathrm{y} 54 & \mathrm{z} 54\end{array}$

$\begin{array}{llllllll}55 & \mathrm{H} & 34 & 23 & 7 & \mathrm{x} 55 & \text { y55 } & \mathrm{z} 55\end{array}$

56 C $35237 \times 56$ y56 z56

$\begin{array}{lllllllll}57 & \mathrm{H} & 35 & 23 & 7 & x 57 & \text { y57 } & \text { z5 }\end{array}$

$\begin{array}{lllllllll}58 & \mathrm{H} & 35 & 23 & 7 & \mathrm{x} 58 & \mathrm{y} 58 & \mathrm{z} 58\end{array}$

59 C $3724 \quad 7 \times 59$ y59 z59

$60 \mathrm{H} 3724 \quad 7 \quad x 60$ y60 z60

$\begin{array}{lllllllll}61 & \mathrm{H} & 37 & 24 & 7 & \mathrm{x} 61 & \mathrm{y} 61 & \mathrm{z} 61\end{array}$

$\begin{array}{llllllll}62 & \mathrm{C} & 38 & 24 & 7 & \mathrm{x} 62 & \text { y62 } & \mathrm{z} 62\end{array}$

$\begin{array}{llllllll}63 & \mathrm{H} & 38 & 24 & 7 & \mathrm{x} 63 & \text { y } 63 & \mathrm{z} 63\end{array}$

$\begin{array}{lllllllll}64 & \mathrm{H} & 38 & 24 & 7 & \mathrm{x} 64 & \mathrm{y} 64 & \mathrm{z} 64\end{array}$

65 H 412618 x65 y65 z65

$\begin{array}{llllllll}66 & \mathrm{H} & 44 & 29 & 21 & \mathrm{x} 66 & \text { y66 } & \text { z } 66\end{array}$

$\begin{array}{lllllllll}67 & \mathrm{C} & 44 & 29 & 21 & \mathrm{x} 67 & \mathrm{y} 67 & \mathrm{z} 67\end{array}$

$\begin{array}{lllllllll}68 & \mathrm{H} & 45 & 30 & 21 & \mathrm{x} 68 & \text { y68 } & \mathrm{z} 68\end{array}$

69 C $473122 \times 69$ y69 z69

$\begin{array}{lllllllll}70 & \mathrm{H} & 47 & 31 & 22 & \mathrm{x} 70 & \text { y } 70 & \text { z } 70\end{array}$

$\begin{array}{lllllllll}71 & \mathrm{H} & 47 & 31 & 22 & \mathrm{x} 71 & \mathrm{y} 71 & \mathrm{z} 71\end{array}$

$\begin{array}{llllllll}72 & \mathrm{H} & 50 & 32 & 22 & \mathrm{x} 72 & \mathrm{y} 72 & \mathrm{z} 72\end{array}$

$\begin{array}{llllllll}73 & \mathrm{H} & 50 & 32 & 22 & x 73 & \text { y73 } & \text { z73 }\end{array}$

$\begin{array}{lllllllll}74 & C & 53 & 34 & 23 & x 74 & y 74 & \text { z } 74\end{array}$

$\begin{array}{llllllll}75 & \mathrm{H} & 53 & 34 & 23 & \mathrm{x} 75 & \mathrm{y} 75 & \mathrm{z} 75\end{array}$

$\begin{array}{lllllllll}76 & \mathrm{H} & 53 & 34 & 23 & \mathrm{x} 76 & \mathrm{y} 76 & \mathrm{z} 76\end{array}$

$\begin{array}{lllllllll}77 & \mathrm{H} & 56 & 35 & 23 & \mathrm{x} 77 & \mathrm{y} 77 & \mathrm{z} 77\end{array}$

$\begin{array}{lllllllll}78 & \mathrm{H} & 56 & 35 & 23 & \mathrm{x} 78 & \text { y78 } & \text { z78 }\end{array}$

$\begin{array}{lllllllll}79 & \mathrm{C} & 59 & 37 & 24 & x 79 & \text { y79 } & \text { z79 }\end{array}$

80 H $59 \begin{array}{lllllll}87 & 24 & x 80 & y 80 & z 80\end{array}$

$\begin{array}{lllllllll}81 & \mathrm{H} & 59 & 37 & 24 & \mathrm{x} 81 & \mathrm{y} 81 & \mathrm{z} 81\end{array}$

$\begin{array}{llllllll}82 & \mathrm{H} & 62 & 38 & 24 & \mathrm{x} 82 & \mathrm{y} 82 & \mathrm{z} 82\end{array}$

$\begin{array}{lllllllll}83 & \mathrm{H} & 62 & 38 & 24 & \mathrm{x} 83 & \mathrm{y} 83 & \mathrm{z} 83\end{array}$

$\begin{array}{lllllllll}84 & \mathrm{H} & 67 & 44 & 29 & \mathrm{x} 84 & \mathrm{y} 84 & \mathrm{z} 84\end{array}$

$\begin{array}{lllllllll}85 & \mathrm{H} & 69 & 47 & 31 & \mathrm{x} 85 & \mathrm{y} 85 & \mathrm{z} 85\end{array}$

$\begin{array}{lllllllll}86 & \mathrm{H} & 69 & 47 & 31 & \mathrm{x} 86 & \mathrm{y} 86 & \mathrm{z} 86\end{array}$

$\begin{array}{lllllllll}87 & \mathrm{H} & 74 & 53 & 34 & \mathrm{x} 87 & \mathrm{y} 87 & \mathrm{z} 87\end{array}$

$\begin{array}{lllllllll}88 & \mathrm{H} & 74 & 53 & 34 & \mathrm{x} 88 & \text { y88 } & \mathrm{z} 88\end{array}$

89 H $795937 \quad x 89$ y89 z89

90 H $795937 \times 90$ y90 z90

END

GEOVAR

$x 10.0$

y1 0.0

z1 0.0

x2 2.44159328611

y2 0.0 


$$
\begin{aligned}
& \text { z2 } 0.0 \\
& \text { ×3 2.45750994732 } \\
& \text { y3 } 171.196434137 \\
& \text { z3 } 0.0 \\
& \text { x4 2.22402156543 } \\
& \text { y4 } 88.7233757281 \\
& \text { z4 } 62.0901356802 \\
& \times 52.38580993124 \\
& \text { y5 } 85.3261154225 \\
& \text { z5 } 339.789675571 \\
& \times 61.90685663845 \\
& \text { y6 } 98.089564725 \\
& \text { z6 } 152.824227759 \\
& \times 72.43360249148 \\
& \text { y7 } 94.5935875419 \\
& \text { z7 } 241.998292492 \\
& \begin{array}{l}
x 83.30387109077 \\
x 86.6180318179
\end{array} \\
& \text { y8 } 66.6180318179 \\
& \text { z8 } 331.50353101 \\
& \times 93.27719055951 \\
& \text { y9 } 103.817925101 \\
& \text { z9 } 349.691018139 \\
& \text { x10 3.24950084194 } \\
& \text { y10 } 50.1068750027 \\
& \text { z10 } 320.098743813 \\
& \text { x11 4.64071810508 } \\
& \text { y11 } 71.2532357026 \\
& \text { z11 335.197735772 } \\
& \text { x12 4.61454362703 } \\
& \text { y12 } 98.2252649791 \\
& \text { z12 } 348.105339696 \\
& \text { x13 } 3.21704359071 \\
& \text { y13 121.492681063 } \\
& \text { z13 } 358.172383559 \\
& \text { x14 } 5.44805580793 \\
& \text { y14 } 63.7199452527 \\
& \text { z14 } 331.279952082 \\
& \text { x15 } 5.19885853473 \\
& \text { y15 } 84.6103009356 \\
& \text { z15 } 342.127719512 \\
& \text { x16 } 6.09064612121 \\
& \text { y16 } 110.205872402 \\
& \text { z16 } 354.372831913 \\
& \text { x17 } 6.28740317159 \\
& \text { y17 } 84.6003146468 \\
& \text { z17 } 342.507621713 \\
& \text { x18 } 1.35238531606 \\
& \text { y18 } 119.126563207 \\
& \text { z18 } 156.310366082 \\
& \text { x19 1.35222711071 } \\
& \begin{array}{ll}
\text { y19 } 122.391981814
\end{array} \\
& \text { z19 } 332.383605397 \\
& \text { x201.10399315308 } \\
& \text { y20 } 116.170105314 \\
& \text { z20 } 141.170321445 \\
& \text { x21 1.45168158071 } \\
& \text { y21 } 133.764559242
\end{aligned}
$$




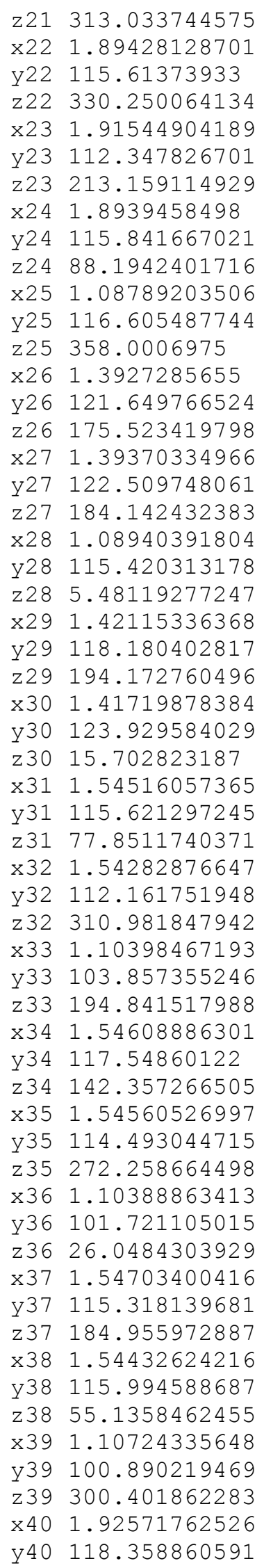




$\begin{array}{ll}\text { z40 } & 181.294112082 \\ \text { x41 } & 1.39354204367 \\ \text { y41 } & 120.784167283 \\ \text { z41 } & 0.630914637947 \\ \text { x42 } & 1.08999882114 \\ \text { y42 } & 119.571442298 \\ \text { z42 } & 179.991937769 \\ \text { x43 } & 1.09269477619 \\ \text { y43 } & 118.71690236 \\ \text { z43 } & 0.670966064204 \\ \text { x44 } & 1.39212492748 \\ \text { y44 } & 121.287818017 \\ \text { z44 } & 180.390483091 \\ \text { x45 } & 1.39274531466 \\ \text { y45 } & 120.623243499 \\ \text { z45 } & 178.660409478 \\ \text { x46 } & 1.08898831211 \\ \text { y46 } & 118.562071071 \\ \text { z46 } & 0.868939643908 \\ \text { x47 } & 1.54071283585 \\ \text { y47 } & 110.61238445 \\ \text { z47 } & 174.7940458 \\ \text { x48 } & 1.09947357735 \\ \text { y48 } & 111.112800276 \\ \text { z48 } & 53.5876302031 \\ \text { x49 } & 1.09972110863 \\ \text { y49 } & 109.02968229 \\ \text { z49 } & 295.519021063 \\ \text { x50 } & 1.53849599427 \\ \text { y50 } & 111.308226327 \\ \text { z50 } & 187.566419571 \\ \text { x51 } & 1.10091254409 \\ \text { y51 } & 111.063899105 \\ \text { z51 } & 310.034167948 \\ \text { x52 } & 1.10119829726 \\ \text { y52 } & 108.4833305 \\ \text { z52 } & 66.9925506986 \\ \text { x53 } & 1.54175279779 \\ \text { y53 } & 110.762631398 \\ \text { z53 } & 190.07962429 \\ \text { x54 } & 1.09781024547 \\ \text { y54 } & 111.650152426 \\ \text { z54 } & 311.100123299 \\ \text { x55 } & 1.10366572072 \\ \text { y55 } & 109.677848057 \\ \text { z55 } & 69.4438861037 \\ \text { x56 } & 1.53794876975 \\ \text { y56 } & 110.958679109 \\ \text { z56 } & 167.268591827 \\ \text { x57 } & 1.09542926861 \\ \text { y57 } & 110.214845486 \\ \text { z57 } & 45.7122689364 \\ \text { x58 } & 1.10259808215 \\ \text { y58 } & 109.240566478 \\ \text { z58 } & 286.987172516 \\ \text { x59 } & 1.54057928787 \\ \text { y59 } & 110.937820091 \\ \end{array}$




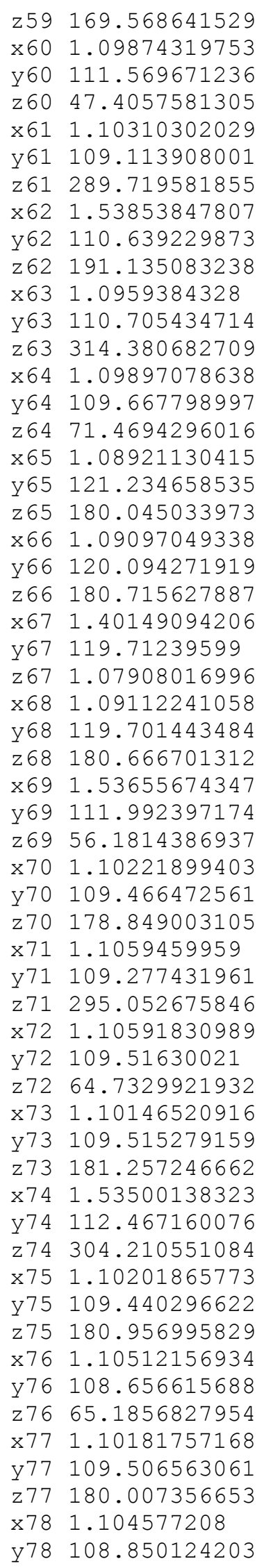




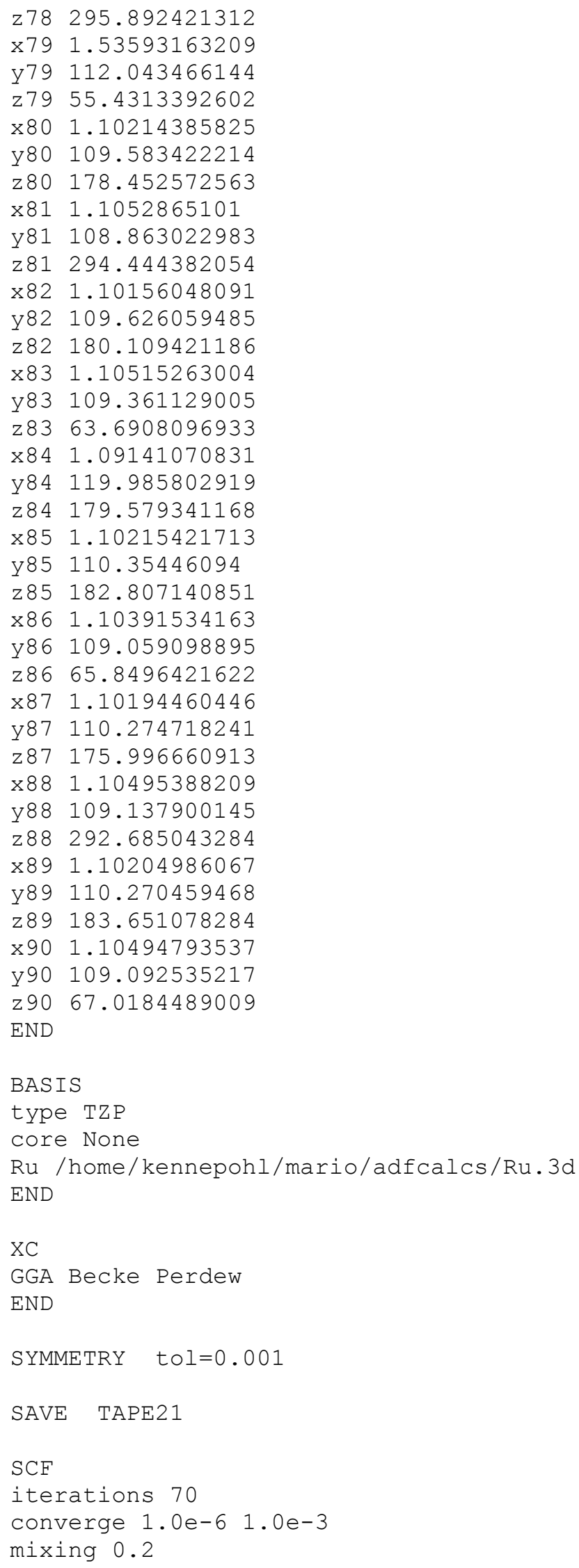


lshift 0.0

diis $\mathrm{n}=10 \quad \mathrm{ok}=0.5 \quad \mathrm{cyc}=5 \quad \mathrm{cx}=5.0 \quad \mathrm{cxx}=10.0$

END

INTEGRATION $3.0 \quad 6.0 \quad 6.0$

A1FIT 10.0

Occupations Keeporbitals $=100$

eor 
S20: ADF input file for ground state calculation on compound $\mathbf{3 b}$. Input geometry was previously optimized (Up to an integration level of 4.0) using the same density functional (Becke and Perdew) and basis set parameters (TZP). A ruthenium $3 d$ core was used in this calculation. Complete output for this calculation will be provided upon request (Pierre@chem.ubc.ca.)

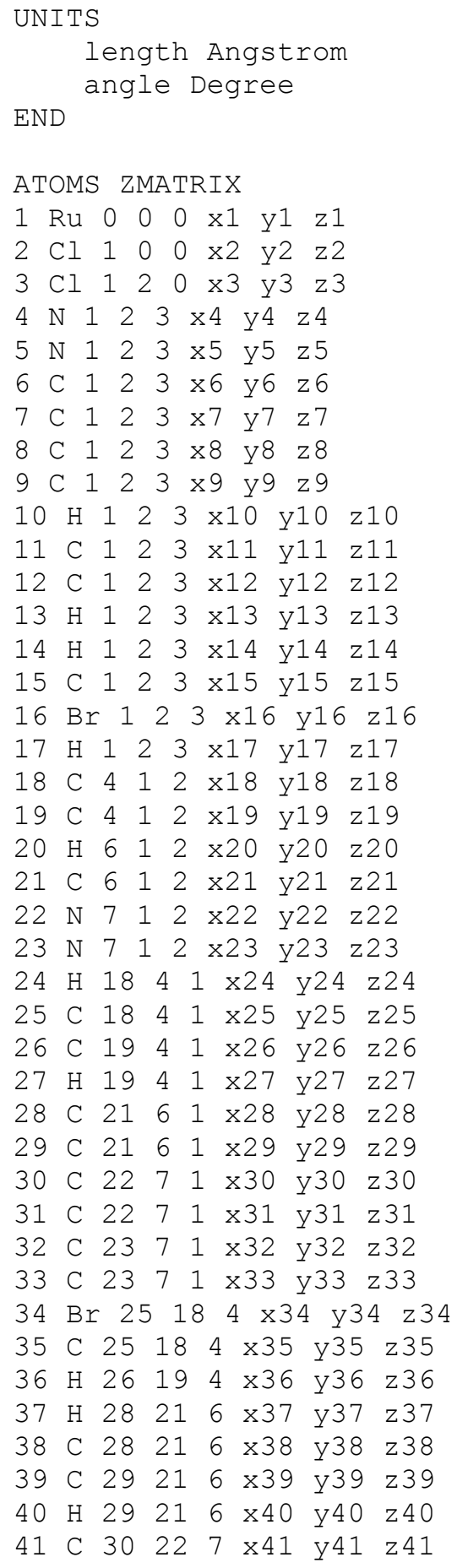


$\begin{array}{llllllll}42 & \mathrm{C} & 30 & 22 & 7 & \mathrm{x} 42 & \text { y42 } & \mathrm{z} 42\end{array}$

$\begin{array}{lllllllll}43 & \mathrm{H} & 31 & 22 & 7 & \mathrm{x} 43 & \mathrm{y} 43 & \mathrm{z} 43\end{array}$

$\begin{array}{lllllllll}44 & \mathrm{H} & 31 & 22 & 7 & \mathrm{x} 44 & \mathrm{y} 44 & \mathrm{z} 44\end{array}$

$\begin{array}{lllllllll}45 & \text { C } & 32 & 23 & 7 & x 45 & \text { y45 } & \text { z45 }\end{array}$

46 C $32 \quad 23 \quad 7 \quad x 46$ y46 z46

$\begin{array}{lllllllll}47 & \mathrm{H} & 33 & 23 & 7 & \mathrm{x} 47 & \mathrm{y} 47 & \mathrm{z} 47\end{array}$

$\begin{array}{llllllll}48 & \mathrm{H} & 33 & 23 & 7 & \mathrm{x} 48 & \mathrm{y} 48 & \mathrm{z} 48\end{array}$

$\begin{array}{llllllll}49 & \mathrm{H} & 35 & 25 & 18 & \times 49 & \mathrm{y} 49 & \mathrm{z} 49\end{array}$

$\begin{array}{llllllllll}50 & \mathrm{H} & 38 & 28 & 21 & \mathrm{x} 50 & \mathrm{y} 50 & \mathrm{z} 50\end{array}$

$\begin{array}{lllllllll}51 & \mathrm{C} & 38 & 28 & 21 & \mathrm{x} 51 & \mathrm{y} 51 & \mathrm{z} 51\end{array}$

$\begin{array}{llllllll}52 & \text { H } & 39 & 29 & 21 & \text { x52 y52 z52 }\end{array}$

$\begin{array}{llllllll}53 & \mathrm{C} & 41 & 30 & 22 & \mathrm{x} 53 & \text { y53 z53 }\end{array}$

$\begin{array}{lllllllll}54 & \mathrm{C} & 41 & 30 & 22 & \mathrm{x} 54 & \mathrm{y} 54 & \mathrm{z} 54\end{array}$

55 C $42 \quad 30 \quad 22 \quad x 55$ y55 z55

56 C $42 \quad 30 \quad 22 \times 56$ y56 z56

$\begin{array}{lllllllll}57 & \mathrm{C} & 45 & 32 & 23 & \times 57 & \mathrm{y} 57 & \mathrm{z} 57\end{array}$

$\begin{array}{lllllllll}58 & C & 45 & 32 & 23 & x 58 & y 58 & \text { z } 58\end{array}$

$\begin{array}{llllllll}59 & \mathrm{C} & 46 & 32 & 23 & \mathrm{x} 59 & \text { y59 z } 59\end{array}$

60 C $4632 \quad 23 \times 60$ y60 z60

$\begin{array}{lllllllll}61 & \mathrm{H} & 51 & 38 & 28 & \mathrm{x} 61 & \mathrm{y} 61 & \mathrm{z} 61\end{array}$

$\begin{array}{lllllllll}62 & \mathrm{H} & 53 & 41 & 30 & \text { x62 } & \text { y62 } & \text { z } 62\end{array}$

63 C 534130 x63 y63 z63

$\begin{array}{lllllllll}64 & \mathrm{H} & 54 & 41 & 30 & \text { x64 } & \text { y64 } & \text { z } 64\end{array}$

$\begin{array}{llllllll}65 & \mathrm{H} & 54 & 41 & 30 & \mathrm{x} 65 & \mathrm{y} 65 & \mathrm{z} 65\end{array}$

$\begin{array}{llllllll}66 & \mathrm{H} & 54 & 41 & 30 & \text { x66 y66 } & \text { z } 66\end{array}$

$\begin{array}{llllllll}67 & \mathrm{H} & 55 & 42 & 30 & \mathrm{x} 67 & \mathrm{y} 67 & \mathrm{z} 67\end{array}$

$\begin{array}{lllllllll}68 & \mathrm{H} & 56 & 42 & 30 & \mathrm{x} 68 & \text { y68 } & \mathrm{z} 68\end{array}$

69 H $56 \quad 4230$ x69 y69 z69

$\begin{array}{lllllllll}70 & \mathrm{H} & 56 & 42 & 30 & \mathrm{x} 70 & \text { y } 70 & \mathrm{z} 70\end{array}$

$\begin{array}{lllllllll}71 & \mathrm{H} & 57 & 45 & 32 & \mathrm{x} 71 & \mathrm{y} 71 & \mathrm{z} 71\end{array}$

$\begin{array}{llllllll}72 & \mathrm{C} & 57 & 45 & 32 & \mathrm{x} 72 & \mathrm{y} 72 & \mathrm{z} 72\end{array}$

$\begin{array}{llllllll}73 & \mathrm{H} & 58 & 45 & 32 & x 73 & y 73 & \text { z } 73\end{array}$

$\begin{array}{llllllll}74 & \mathrm{H} & 58 & 45 & 32 & \mathrm{x} 74 & \mathrm{y} 74 & \mathrm{z} 74\end{array}$

$\begin{array}{llllllll}75 & \mathrm{H} & 58 & 45 & 32 & \mathrm{x} 75 & \mathrm{y} 75 & \mathrm{z} 75\end{array}$

$\begin{array}{lllllllll}76 & \text { H } & 59 & 46 & 32 & x 76 & \text { y } 76 & \text { z } 76\end{array}$

$\begin{array}{llllllll}77 & \mathrm{H} & 60 & 46 & 32 & \mathrm{x} 77 & \text { y77 } & \mathrm{z} 77\end{array}$

$\begin{array}{llllllll}78 & \mathrm{H} & 60 & 46 & 32 & \mathrm{x} 78 & \mathrm{y} 78 & \mathrm{z} 78\end{array}$

$\begin{array}{lllllllll}79 & \mathrm{H} & 60 & 46 & 32 & x 79 & \text { y79 } & \text { z } 79\end{array}$

80 C $63 \quad 53 \quad 41 \quad x 80$ y80 z80

$\begin{array}{llllllllll}81 & \mathrm{C} & 72 & 57 & 45 & \mathrm{x} 81 & \mathrm{y} 81 & \mathrm{z} 81\end{array}$

$\begin{array}{lllllllll}82 & \mathrm{H} & 80 & 63 & 53 & \mathrm{x} 82 & \text { y82 } & \mathrm{z} 82\end{array}$

$\begin{array}{lllllllll}83 & \mathrm{H} & 80 & 63 & 53 & \mathrm{x} 83 & \text { y83 } & \mathrm{z} 83\end{array}$

$\begin{array}{lllllllll}84 & \mathrm{H} & 80 & 63 & 53 & \mathrm{x} 84 & \mathrm{y} 84 & \mathrm{z} 84\end{array}$

85 H $81 \quad 7257 \quad x 85$ y 85 z 85

$\begin{array}{lllllllll}86 & \mathrm{H} & 81 & 72 & 57 & \mathrm{x} 86 & \mathrm{y} 86 & \mathrm{z} 86\end{array}$

$\begin{array}{llllllllll}87 & \mathrm{H} & 81 & 72 & 57 & \mathrm{x} 87 & \mathrm{y} 87 & \mathrm{z} 87\end{array}$

END

GEOVAR

$x 10.0$

y1 0.0

z1 0.0

x2 2.48614641965

y2 0.0

z2 0.0

×3 2.45899491966

y3 174.383520269 
z3 0.0

$\times 42.26179891735$

y4 91.1514148641

z4 113.135677729

$\times 52.43068919177$

y5 87.3866002806

z5 37.741386843

×6 1.90778348845

y6 85.3108371339

z6 199.520971158

x7 2.08060351855

y7 87.7229339364

z7 294.895526007

$x 83.30826874801$

y8 67.4645273747

z8 44.0835011115

$\times 93.33148341827$

y9 107.683872013

z9 37.1454164019

x10 3.23179240361

y10 48.3774089094

z10 46.1311249097

x11 4.64077420119

y11 73.1111587485

z11 46.2570966249

x12 4.64975702407

y12 102.348797292

z12 41.2144254317

x13 3.29287832722

y13 125.972019304

z13 31.5674650925

x14 5.43301702576

y14 64.8609605865

z14 48.6636430145

x15 5.21376792801

y15 87.9069853844

z15 44.6988357834

$\times 166.1390574375$

y16 115.550214831

z16 40.0355752436

x17 6.29746330336

y17 88.1075213489

z17 45.7930128768

x18 1.35072621072

y18 119.611795446

z18 12.6616939143

x19 1.34926338868

y19 121.89289931

z19 194.109887745

×201.10098544603

y20 113.534343014

z20 18.5055018826

x21 1.45820474177

y21 134.848748237

z21 214.293876496

x22 1.36891001057

y22 126.925595508 


\begin{tabular}{|c|c|}
\hline z22 & 94.6910365635 \\
\hline$\times 23$ & 1.37108793923 \\
\hline y23 & 126.890031157 \\
\hline 23 & 277.658366424 \\
\hline$\times 24$ & 1.09117923029 \\
\hline y24 & 116.215611669 \\
\hline z24 & 0.640216853997 \\
\hline x25 & 1.39270801523 \\
\hline & 121.442642194 \\
\hline 25 & 182.061287935 \\
\hline 26 & 1.39408738611 \\
\hline y26 & 122.528290285 \\
\hline z26 & 178.476426759 \\
\hline x27 & 1.09056031192 \\
\hline y27 & 115.366074264 \\
\hline z27 & 358.103721055 \\
\hline$\times 28$ & 1.42075372605 \\
\hline y28 & 117.621833893 \\
\hline z28 & 160.731958143 \\
\hline$\times 29$ & 1.41626436538 \\
\hline y29 & 124.706630615 \\
\hline z29 & 339.738339782 \\
\hline$\times 30$ & 1.4415483287 \\
\hline y30 & 131.783717363 \\
\hline z30 & 1.77739999439 \\
\hline ×31 & 1.48177728955 \\
\hline y31 & 113.5846445 \\
\hline z31 & 187.040761653 \\
\hline x32 & 1.43982829118 \\
\hline y32 & 131.493944737 \\
\hline z32 & 349.835522442 \\
\hline x33 & 1.48182728017 \\
\hline y33 & 113.58558649 \\
\hline z33 & 180.975982278 \\
\hline$\times 34$ & 1.92884509043 \\
\hline y34 & 118.850435089 \\
\hline z34 & 178.689543905 \\
\hline ×35 & 1.39331229202 \\
\hline y35 & 120.787614984 \\
\hline z35 & 359.205674559 \\
\hline x36 & 1.08986368048 \\
\hline y36 & 119.675036886 \\
\hline z36 & 179.875638698 \\
\hline$\times 37$ & 1.09244597937 \\
\hline y37 & 118.651697364 \\
\hline z37 & 359.024698975 \\
\hline ×38 & 1.39345603478 \\
\hline y38 & 121.42681575 \\
\hline z38 & 179.058964556 \\
\hline$\times 3$ & 1.39356070102 \\
\hline y3 & 120.774004324 \\
\hline z3 & 181.85912283 \\
\hline$\times 40$ & 1.08945956578 \\
\hline y 40 & 118.553756039 \\
\hline z 40 & 0.0707310351408 \\
\hline & 1.41299516481 \\
\hline & 118.429055465 \\
\hline
\end{tabular}




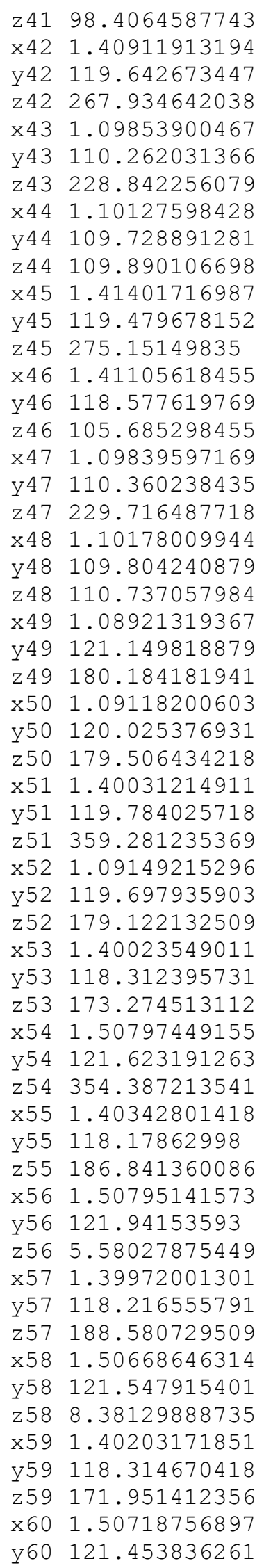




$$
\begin{array}{ll}
\text { z60 } & 351.950107942 \\
\text { x61 } & 1.09168340365 \\
\text { y61 } & 120.086446386 \\
\text { z61 } & 180.491731501 \\
\text { x62 } & 1.09322292721 \\
\text { y62 } & 118.573464827 \\
\text { z62 } & 179.02204447 \\
\text { x63 } & 1.40180953446 \\
\text { y63 } & 122.070634553 \\
\text { z63 } & 358.552503843 \\
\text { x64 } & 1.09718503967 \\
\text { y64 } & 110.453826097 \\
\text { z64 } & 181.421306351 \\
\text { x65 } & 1.10099301962 \\
\text { y65 } & 111.472245847 \\
\text { z65 } & 61.8870870929 \\
\text { x66 } & 1.09926125206 \\
\text { y66 } & 111.219068874 \\
\text { z66 } & 302.106165294 \\
\text { x67 } & 1.09315241527 \\
\text { y67 } & 118.500423524 \\
\text { z67 } & 180.979165829 \\
\text { x68 } & 1.09752495177 \\
\text { y68 } & 110.436064996 \\
\text { z68 } & 168.121250015 \\
\text { x69 } & 1.0989880844 \\
\text { y69 } & 112.140731019 \\
\text { z69 } & 46.9585875015 \\
\text { x70 } & 1.10214157577 \\
\text { y70 } & 111.149489889 \\
\text { z70 } & 286.700802943 \\
\text { x71 } & 1.09293528817 \\
\text { y71 } & 118.447951681 \\
\text { z71 } & 180.537243561 \\
\text { x72 } & 1.40139091189 \\
\text { y72 } & 122.152102471 \\
\text { z72 } & 0.659576355702 \\
\text { x73 } & 1.10137150635 \\
\text { y73 } & 111.582615727 \\
\text { z73 } & 296.231443859 \\
\text { x74 } & 1.09687999724 \\
\text { y74 } & 110.50505137 \\
\text { z74 } & 176.569582237 \\
\text { x75 } & 1.0997198252 \\
\text { y75 } & 111.506170334 \\
\text { z75 } & 55.7903412107 \\
\text { x76 } & 1.09319471631 \\
\text { y76 } & 118.576932535 \\
\text { z76 } & 179.174363557 \\
\text { x77 } & 1.1002893075 \\
\text { y77 } & 111.422672328 \\
\text { z77 } & 306.712088808 \\
\text { x78 } & 1.09723418182 \\
\text { y78 } & 110.537074014 \\
\text { z78 } & 185.649978603 \\
\text { x79 } & 1.10133102358 \\
\text { y79 } & 111.53448821 \\
\end{array}
$$




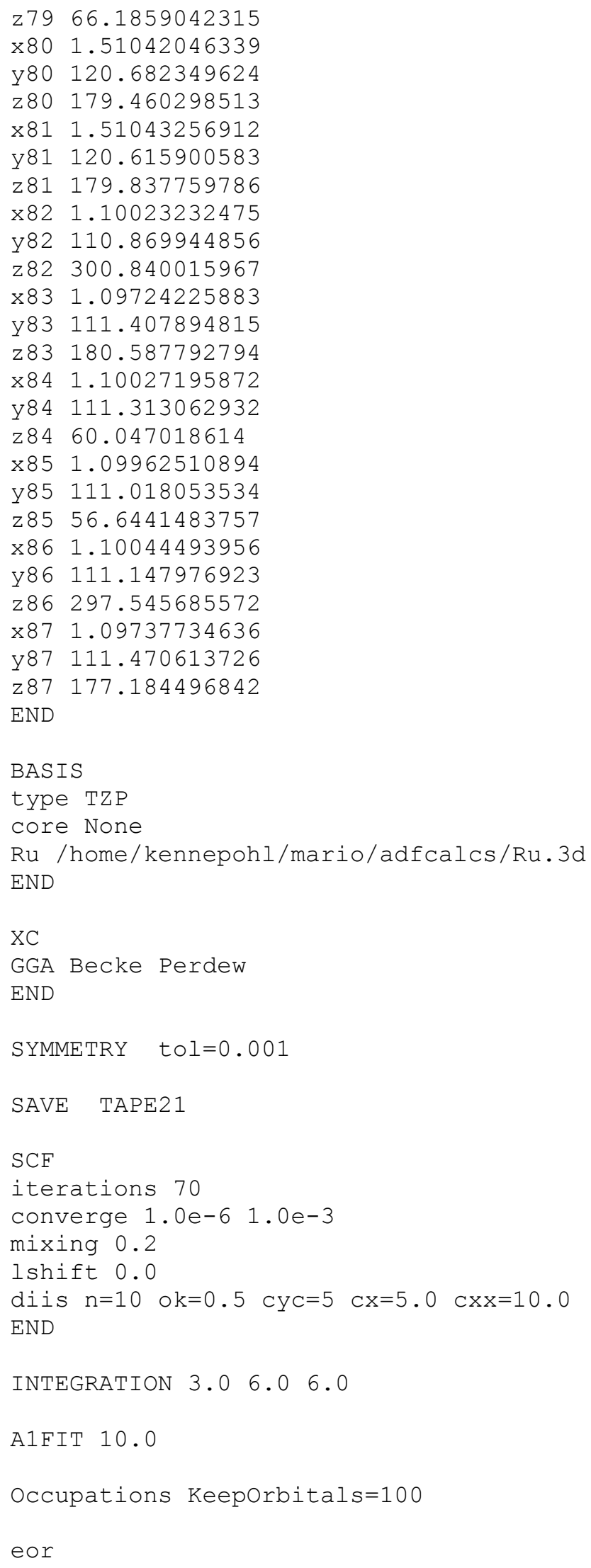


S21: Mulliken decomposition of valence Kohn-Sham molecular orbitals for complex 1a. Table items are color-coded such that the importance of contributions are represented by different shades of red. Numbers in red are negative Mulliken populations.

\begin{tabular}{|c|c|c|c|c|c|c|c|c|c|c|c|c|c|c|c|c|c|c|c|c|c|c|c|}
\hline \multirow{3}{*}{$\mathrm{E}(\mathrm{eV})$} & \multirow{3}{*}{ Orbital } & \multirow{3}{*}{ Occ } & \multicolumn{21}{|c|}{ Fragment Decomposition } \\
\hline & & & \multicolumn{4}{|c|}{$\mathrm{Ru}$} & \multicolumn{3}{|c|}{$\mathrm{Cl}^{1}$} & \multicolumn{3}{|c|}{$\mathrm{Cl}^{2}$} & \multicolumn{4}{|c|}{$\mathrm{PR}_{3}{ }^{1}$} & \multicolumn{4}{|c|}{$\mathrm{PR}_{3}{ }^{2}$} & \multicolumn{3}{|c|}{$\mathrm{CHPh}$} \\
\hline & & & Total & $4 d$ & $5 s$ & $5 p$ & Total & $3 \mathrm{~s}$ & $3 p$ & Total & $3 \mathrm{~s}$ & $3 p$ & Total & P 3s & P 3p & $\mathrm{R}_{3}$ & Total & P 3s & P $3 p$ & $\mathrm{R}_{3}$ & Total & $\mathrm{CH}$ & $\mathrm{Ph}$ \\
\hline 0.68 & $220 \mathrm{~A}$ & 0 & 0.000 & 0.000 & 0.000 & 0.000 & 0.000 & 0.000 & 0.000 & 0.000 & 0.000 & 0.000 & 0.141 & 0.000 & 0.000 & 0.141 & 0.614 & 0.000 & 0.012 & 0.603 & 0.235 & 0.014 & 0.221 \\
\hline 0.58 & $219 \mathrm{~A}$ & 0 & 0.000 & 0.000 & 0.000 & 0.000 & 0.000 & 0.000 & 0.000 & 0.000 & 0.000 & 0.000 & 0.658 & 0.000 & 0.109 & 0.538 & 0.200 & 0.000 & 0.012 & 0.188 & 0.000 & 0.000 & 0.000 \\
\hline 0.55 & $218 \mathrm{~A}$ & 0 & 0.011 & 0.000 & 0.011 & 0.000 & 0.000 & 0.000 & 0.000 & 0.000 & 0.000 & 0.000 & 0.829 & 0.000 & 0.068 & 0.748 & 0.013 & 0.000 & 0.000 & 0.013 & 0.000 & 0.000 & 0.000 \\
\hline 0.40 & $217 \mathrm{~A}$ & 0 & 0.011 & 0.011 & 0.000 & 0.000 & 0.000 & 0.000 & 0.000 & 0.000 & 0.000 & 0.000 & 0.249 & 0.000 & 0.121 & 0.115 & 0.489 & 0.000 & 0.042 & 0.531 & 0.112 & 0.000 & 0.112 \\
\hline 0.35 & $216 \mathrm{~A}$ & 0 & 0.062 & 0.000 & 0.062 & 0.000 & 0.000 & 0.000 & 0.000 & 0.000 & 0.000 & 0.000 & 0.362 & 0.000 & 0.003 & 0.360 & 0.457 & 0.000 & 0.030 & 0.487 & 0.011 & 0.000 & 0.011 \\
\hline 0.26 & $215 \mathrm{~A}$ & 0 & 0.156 & 0.000 & 0.156 & 0.000 & 0.000 & 0.000 & 0.000 & 0.000 & 0.000 & 0.000 & 0.282 & 0.000 & 0.036 & 0.246 & 0.187 & 0.000 & 0.023 & 0.164 & 0.312 & 0.015 & 0.326 \\
\hline 0.12 & $214 \mathrm{~A}$ & 0 & 0.189 & 0.019 & 0.170 & 0.000 & 0.000 & 0.000 & 0.000 & 0.012 & 0.012 & 0.000 & 0.260 & 0.000 & 0.090 & 0.171 & 0.360 & 0.000 & 0.019 & 0.341 & 0.000 & 0.000 & 0.000 \\
\hline 0.07 & $213 \mathrm{~A}$ & 0 & 0.064 & 0.012 & 0.013 & 0.039 & 0.015 & 0.015 & 0.000 & 0.000 & 0.000 & 0.000 & 0.302 & 0.000 & 0.129 & 0.151 & 0.330 & 0.000 & 0.042 & 0.288 & 0.013 & 0.013 & 0.000 \\
\hline-0.01 & $212 \mathrm{~A}$ & 0 & 0.103 & 0.000 & 0.103 & 0.000 & 0.000 & 0.000 & 0.000 & 0.000 & 0.000 & 0.000 & 0.437 & 0.011 & 0.054 & 0.502 & 0.185 & 0.000 & 0.000 & 0.185 & 0.050 & 0.000 & 0.050 \\
\hline-0.08 & $211 \mathrm{~A}$ & 0 & 0.237 & 0.000 & 0.237 & 0.000 & 0.000 & 0.000 & 0.000 & 0.000 & 0.000 & 0.000 & 0.541 & 0.013 & 0.011 & 0.544 & 0.001 & 0.000 & 0.000 & 0.001 & 0.022 & 0.000 & 0.022 \\
\hline-0.37 & $210 \mathrm{~A}$ & 0 & 0.039 & 0.039 & 0.000 & 0.000 & 0.000 & 0.000 & 0.000 & 0.000 & 0.000 & 0.000 & 0.000 & 0.000 & 0.000 & 0.000 & 0.000 & 0.000 & 0.000 & 0.000 & 0.785 & 0.205 & 0.580 \\
\hline-1.30 & $209 \mathrm{~A}$ & 0 & 0.000 & 0.000 & 0.000 & 0.000 & 0.000 & 0.000 & 0.000 & 0.000 & 0.000 & 0.000 & 0.000 & 0.000 & 0.000 & 0.000 & 0.000 & 0.000 & 0.000 & 0.000 & 0.867 & 0.000 & 0.867 \\
\hline-1.68 & $208 \mathrm{~A}$ & 0 & 0.479 & 0.420 & 0.017 & 0.042 & 0.047 & 0.000 & 0.047 & 0.045 & 0.000 & 0.045 & 0.142 & 0.057 & 0.069 & 0.016 & 0.136 & 0.045 & 0.091 & 0.000 & 0.047 & 0.047 & 0.000 \\
\hline-1.96 & $207 \mathrm{~A}$ & 0 & 0.536 & 0.418 & 0.000 & 0.118 & 0.072 & 0.000 & 0.072 & 0.080 & 0.000 & 0.080 & 0.027 & 0.000 & 0.027 & 0.000 & 0.023 & 0.000 & 0.023 & 0.000 & 0.127 & 0.127 & 0.000 \\
\hline-2.90 & $206 \mathrm{~A}$ & 0 & 0.241 & 0.241 & 0.000 & 0.000 & 0.000 & 0.000 & 0.000 & 0.000 & 0.000 & 0.000 & 0.014 & 0.000 & 0.014 & 0.000 & 0.000 & 0.000 & 0.000 & 0.000 & 0.646 & 0.287 & 0.359 \\
\hline-3.98 & $205 \mathrm{~A}$ & 2 & 0.720 & 0.720 & 0.000 & 0.000 & 0.103 & 0.000 & 0.103 & 0.090 & 0.000 & 0.090 & 0.000 & 0.000 & 0.000 & 0.000 & 0.000 & 0.000 & 0.000 & 0.000 & 0.046 & 0.046 & 0.000 \\
\hline-4.13 & $204 \mathrm{~A}$ & 2 & 0.668 & 0.668 & 0.000 & 0.000 & 0.130 & 0.000 & 0.130 & 0.144 & 0.000 & 0.144 & 0.000 & 0.000 & 0.000 & 0.000 & 0.000 & 0.000 & 0.000 & 0.000 & 0.000 & 0.000 & 0.000 \\
\hline-5.22 & $203 \mathrm{~A}$ & 2 & 0.011 & 0.000 & 0.000 & 0.011 & 0.164 & 0.000 & 0.164 & 0.237 & 0.000 & 0.237 & 0.160 & 0.025 & 0.113 & 0.022 & 0.189 & 0.025 & 0.148 & 0.017 & 0.031 & 0.019 & 0.012 \\
\hline-5.62 & $202 \mathrm{~A}$ & 2 & 0.380 & 0.380 & 0.000 & 0.000 & 0.076 & 0.000 & 0.076 & 0.062 & 0.000 & 0.062 & 0.000 & 0.000 & 0.000 & 0.000 & 0.000 & 0.000 & 0.000 & 0.000 & 0.311 & 0.058 & 0.253 \\
\hline-5.95 & $201 \mathrm{~A}$ & 2 & 0.036 & 0.011 & 0.000 & 0.025 & 0.147 & 0.000 & 0.147 & 0.652 & 0.000 & 0.652 & 0.000 & 0.000 & 0.000 & 0.000 & 0.000 & 0.000 & 0.000 & 0.000 & 0.000 & 0.000 & 0.000 \\
\hline-6.10 & $200 \mathrm{~A}$ & 2 & 0.058 & 0.058 & 0.000 & 0.000 & 0.591 & 0.000 & 0.591 & 0.134 & 0.000 & 0.134 & 0.000 & 0.000 & 0.000 & 0.000 & 0.000 & 0.000 & 0.000 & 0.000 & 0.000 & 0.000 & 0.000 \\
\hline-6.21 & $199 \mathrm{~A}$ & 2 & 0.121 & 0.093 & 0.000 & 0.029 & 0.243 & 0.000 & 0.243 & 0.067 & 0.000 & 0.067 & 0.044 & 0.000 & 0.022 & 0.022 & 0.137 & 0.000 & 0.043 & 0.094 & 0.022 & 0.000 & 0.022 \\
\hline-6.28 & $198 \mathrm{~A}$ & 2 & 0.116 & 0.104 & 0.000 & 0.012 & 0.092 & 0.000 & 0.092 & 0.338 & 0.000 & 0.338 & 0.131 & 0.000 & 0.023 & 0.108 & 0.022 & 0.000 & 0.022 & 0.000 & 0.000 & 0.000 & 0.000 \\
\hline-6.33 & $197 \mathrm{~A}$ & 2 & 0.000 & 0.000 & 0.000 & 0.000 & 0.046 & 0.000 & 0.046 & 0.029 & 0.000 & 0.029 & 0.000 & 0.000 & 0.000 & 0.000 & 0.322 & 0.000 & 0.055 & 0.267 & 0.249 & 0.000 & 0.249 \\
\hline
\end{tabular}




\begin{tabular}{|c|c|c|c|c|c|c|c|c|c|c|c|c|c|c|c|c|c|c|c|c|c|c|c|}
\hline-6.42 & $196 \mathrm{~A}$ & 2 & 0.000 & 0.000 & 0.000 & 0.000 & 0.048 & 0.000 & 0.048 & 0.000 & 0.000 & 0.000 & .000 & 0.000 & 0.000 & 0.000 & 0.054 & 0.000 & 0.015 & 0.039 & 0.549 & 0.000 & 0.549 \\
\hline-6.46 & $195 \mathrm{~A}$ & 2 & 0.029 & 0.029 & 0.000 & 0.000 & 0.000 & 0.000 & 0.000 & 0.043 & 0.000 & 0.043 & 0.111 & 0.000 & 0.042 & 0.069 & 0.250 & 0.000 & 0.040 & 0.210 & 0.000 & 0.000 & 0.000 \\
\hline-6.48 & $194 \mathrm{~A}$ & 2 & 0.041 & 0.041 & 0.000 & 0.000 & 0.021 & 0.000 & 0.021 & 0.051 & 0.000 & 0.051 & .015 & 0.000 & 0.015 & 0.000 & 0.297 & 0.000 & 0.045 & 0.252 & 0.000 & 0.000 & 0.000 \\
\hline 6.58 & $193 \mathrm{~A}$ & 2 & 0.031 & 0.031 & 0.000 & 0.000 & 0.069 & 0.000 & 0.069 & 0.011 & 0.000 & 0.011 & 0.493 & 0.000 & 0.089 & 0.404 & 0.000 & 0.000 & 0.000 & 0.000 & 0.000 & 0.000 & 0.000 \\
\hline-6.70 & $192 \mathrm{~A}$ & 2 & 0.080 & 0.053 & 0.000 & 0.027 & 0.257 & 0.000 & 0.257 & 0.282 & 0.000 & 0.282 & 0.011 & 0.000 & 0.011 & 0.000 & 0.024 & 0.000 & 0.000 & 0.024 & 0.000 & 0.000 & 0.000 \\
\hline-6.77 & $191 \mathrm{~A}$ & 2 & 0.024 & 0.024 & 0.000 & 0.000 & 0.011 & 0.000 & 0.011 & 0.000 & 0.000 & 0.000 & 0.459 & 0.000 & 0.000 & 0.459 & 0.000 & 0.000 & 0.000 & 0.000 & 0.000 & 0.000 & 0.000 \\
\hline-6.84 & $190 \mathrm{~A}$ & 2 & 0.031 & 0.031 & 0.000 & 0.000 & 0.025 & 0.000 & 0.025 & 0.000 & 0.000 & 0.000 & 0.424 & 0.000 & 0.021 & 0.403 & 0.011 & 0.000 & 0.011 & 0.000 & 0.000 & 0.000 & 0.000 \\
\hline-6.87 & $189 \mathrm{~A}$ & 2 & 0.010 & 0.010 & 0.000 & 0.000 & 0.015 & 0.000 & 0.015 & 0.013 & 0.000 & 0.013 & 0.072 & 0.000 & 0.000 & 0.072 & 0.456 & 0.000 & 0.000 & 0.456 & 0.000 & 0.000 & 0.000 \\
\hline-6.89 & $188 \mathrm{~A}$ & 2 & 0.000 & 0.000 & 0.000 & 0.000 & 0.000 & 0.000 & 0.000 & 0.038 & 0.000 & 0.038 & 0.292 & 0.000 & 0.018 & 0.273 & 0.214 & 0.000 & 0.000 & 0.214 & 0.000 & 0.000 & 0.000 \\
\hline-6.94 & $187 \mathrm{~A}$ & 2 & 0.000 & 0.000 & 0.000 & 0.000 & 0.000 & 0.000 & 0.000 & 0.016 & 0.000 & 0.016 & 0.148 & 0.000 & 0.000 & 0.148 & 0.281 & 0.000 & 0.000 & 0.281 & 0.024 & 0.000 & 0.024 \\
\hline-7.03 & $186 \mathrm{~A}$ & 2 & 0.000 & 0.000 & 0.000 & 0.000 & 0.022 & 0.000 & 0.022 & 0.000 & 0.000 & 0.000 & 0.286 & 0.000 & 0.000 & 0.286 & 0.165 & 0.000 & 0.000 & 0.165 & 0.000 & 0.000 & 0.000 \\
\hline-7.06 & $185 \mathrm{~A}$ & 2 & 0.000 & 0.000 & 0.000 & 0.000 & 0.016 & 0.000 & 0.016 & 0.000 & 0.000 & 0.000 & 0.054 & 0.000 & 0.000 & 0.054 & 0.465 & 0.000 & 0.022 & 0.444 & 0.000 & 0.000 & 0.000 \\
\hline-7.09 & $184 \mathrm{~A}$ & 2 & 0.000 & 0.000 & 0.000 & 0.000 & 0.000 & 0.000 & 0.000 & 0.000 & 0.000 & 0.000 & 0.165 & 0.000 & 0.000 & 0.165 & 0.285 & 0.000 & 0.011 & 0.275 & 0.000 & 0.000 & 0.000 \\
\hline-7.10 & $183 \mathrm{~A}$ & 2 & 0.000 & 0.000 & 0.000 & 0.000 & 0.022 & 0.000 & 0.022 & 0.000 & 0.000 & 0.000 & 0.209 & 0.000 & 0.000 & 0.209 & 0.173 & 0.000 & 0.000 & 0.173 & 0.000 & 0.000 & 0.000 \\
\hline-7.13 & $182 \mathrm{~A}$ & 2 & 0.016 & 0.016 & 0.000 & 0.000 & 0.000 & 0.000 & 0.000 & 0.000 & 0.000 & 0.000 & 0.168 & 0.000 & 0.047 & 0.122 & 0.293 & 0.000 & 0.000 & 0.293 & 0.000 & 0.000 & 0.000 \\
\hline-7.16 & $181 \mathrm{~A}$ & 2 & 0.000 & 0.000 & 0.000 & 0.000 & 0.012 & 0.000 & 0.012 & 0.021 & 0.000 & 0.021 & 0.039 & 0.000 & 0.000 & 0.039 & 0.451 & 0.000 & 0.017 & 0.433 & 0.000 & 0.000 & 0.000 \\
\hline-7.18 & $180 \mathrm{~A}$ & 2 & 0.022 & 0.022 & 0.000 & 0.000 & 0.011 & 0.000 & 0.011 & 0.000 & 0.000 & 0.000 & 0.587 & 0.000 & 0.014 & 0.573 & 0.000 & 0.000 & 0.000 & 0.000 & 0.000 & 0.000 & 0.000 \\
\hline-7.25 & $179 \mathrm{~A}$ & 2 & 0.054 & 0.054 & 0.000 & 0.000 & 0.015 & 0.000 & 0.015 & 0.000 & 0.000 & 0.000 & 0.132 & 0.000 & 0.000 & 0.132 & 0.146 & 0.000 & 0.021 & 0.126 & 0.241 & 0.069 & 0.172 \\
\hline-7.41 & $178 \mathrm{~A}$ & 2 & 0.047 & 0.047 & 0.000 & 0.000 & 0.000 & 0.000 & 0.000 & 0.000 & 0.000 & 0.000 & 0.013 & 0.000 & 0.013 & 0.000 & 0.167 & 0.000 & 0.017 & 0.149 & 0.270 & 0.090 & 0.181 \\
\hline-7.54 & $177 \mathrm{~A}$ & 2 & 0.083 & 0.056 & 0.027 & 0.000 & 0.250 & 0.000 & 0.250 & 0.166 & 0.000 & 0.166 & 0.024 & 0.000 & 0.014 & 0.010 & 0.000 & 0.000 & 0.000 & 0.000 & 0.154 & 0.143 & 0.011 \\
\hline-7.61 & $176 \mathrm{~A}$ & 2 & 0.043 & 0.043 & 0.000 & 0.000 & 0.038 & 0.000 & 0.038 & 0.040 & 0.000 & 0.040 & 0.236 & 0.018 & 0.000 & 0.218 & 0.132 & 0.000 & 0.000 & 0.132 & 0.000 & 0.000 & 0.000 \\
\hline-7.72 & $175 \mathrm{~A}$ & 2 & 0.150 & 0.150 & 0.000 & 0.000 & 0.048 & 0.000 & 0.048 & 0.051 & 0.000 & 0.051 & 0.011 & 0.000 & 0.011 & 0.000 & 0.191 & 0.013 & 0.011 & 0.168 & 0.093 & 0.071 & 0.022 \\
\hline-7.87 & $174 \mathrm{~A}$ & 2 & 0.215 & 0.215 & 0.000 & 0.000 & 0.029 & 0.000 & 0.029 & 0.023 & 0.000 & 0.023 & 0.016 & 0.000 & 0.016 & 0.000 & 0.012 & 0.000 & 0.012 & 0.000 & 0.241 & 0.116 & 0.125 \\
\hline-8.10 & $173 \mathrm{~A}$ & 2 & 0.000 & 0.000 & 0.000 & 0.000 & 0.000 & 0.000 & 0.000 & 0.000 & 0.000 & 0.000 & 0.689 & 0.000 & 0.000 & 0.689 & 0.000 & 0.000 & 0.000 & 0.000 & 0.000 & 0.000 & 0.000 \\
\hline-8.16 & $172 \mathrm{~A}$ & 2 & 0.000 & 0.000 & 0.000 & 0.000 & 0.000 & 0.000 & 0.000 & 0.000 & 0.000 & 0.000 & 0.513 & 0.000 & 0.015 & 0.498 & 0.040 & 0.000 & 0.000 & 0.040 & 0.000 & 0.000 & 0.000 \\
\hline-8.16 & $171 \mathrm{~A}$ & 2 & 0.000 & 0.000 & 0.000 & 0.000 & 0.000 & 0.000 & 0.000 & 0.000 & 0.000 & 0.000 & 0.000 & 0.000 & 0.000 & 0.000 & 0.552 & 0.000 & 0.029 & 0.523 & 0.000 & 0.000 & 0.000 \\
\hline-8.18 & $170 \mathrm{~A}$ & 2 & 0.000 & 0.000 & 0.000 & 0.000 & 0.000 & 0.000 & 0.000 & 0.000 & 0.000 & 0.000 & 0.000 & 0.000 & 0.000 & 0.000 & 0.606 & 0.000 & 0.000 & 0.606 & 0.000 & 0.000 & 0.000 \\
\hline
\end{tabular}


S22: Mulliken decomposition of valence Kohn-Sham molecular orbitals for complex 1b. Table items are color-coded such that the importance of contributions are represented by different shades of red. Numbers in red are negative Mulliken populations.

\begin{tabular}{|c|c|c|c|c|c|c|c|c|c|c|c|c|c|c|c|c|c|c|c|c|c|c|c|}
\hline \multirow{3}{*}{$E(e V)$} & \multirow{3}{*}{ Orbital } & \multirow{3}{*}{ Occ } & \multicolumn{21}{|c|}{ Fragment Decomposition } \\
\hline & & & \multicolumn{4}{|c|}{ Ru } & \multicolumn{3}{|c|}{$\mathrm{Cl}^{1}$} & \multicolumn{3}{|c|}{$\mathbf{C l}^{2}$} & \multicolumn{4}{|c|}{$\mathbf{P R}_{3}$} & \multicolumn{4}{|c|}{ NHC } & \multicolumn{3}{|c|}{$\mathrm{CHPh}$} \\
\hline & & & Total & 4d & $5 s$ & $5 p$ & Total & $3 s$ & $3 p$ & Total & $3 s$ & $3 p$ & Total & P 3s & P 3p & $\mathbf{R}_{3}$ & Total & NCN & main & Mes $_{2}$ & Total & $\mathbf{C H}$ & $\mathbf{P h}$ \\
\hline 0.01 & $221 \mathrm{~A}$ & 0 & 0.190 & 0.028 & 0.162 & 0.000 & 0.000 & 0.000 & 0.000 & 0.000 & 0.000 & 0.000 & 0.000 & 0.000 & 0.000 & 0.000 & 0.051 & 0.011 & 0.062 & 0.000 & 0.550 & 0.148 & 0.402 \\
\hline-0.07 & $220 \mathrm{~A}$ & 0 & 0.216 & 0.000 & 0.228 & 0.012 & 0.000 & 0.000 & 0.000 & 0.000 & 0.000 & 0.000 & 0.000 & 0.000 & 0.000 & 0.000 & 0.425 & 0.034 & 0.335 & 0.125 & 0.195 & 0.060 & 0.135 \\
\hline-0.60 & $219 \mathrm{~A}$ & 0 & 0.049 & 0.029 & 0.000 & 0.020 & 0.000 & 0.000 & 0.000 & 0.000 & 0.000 & 0.000 & 0.018 & 0.000 & 0.018 & 0.000 & 0.842 & 0.590 & 0.074 & 0.178 & 0.000 & 0.000 & 0.000 \\
\hline-0.83 & $218 \mathrm{~A}$ & 0 & 0.000 & 0.000 & 0.000 & 0.000 & 0.000 & 0.000 & 0.000 & 0.000 & 0.000 & 0.000 & 0.000 & 0.000 & 0.000 & 0.000 & 0.865 & 0.054 & 0.000 & 0.811 & 0.030 & 0.000 & 0.030 \\
\hline-0.95 & $217 \mathrm{~A}$ & 0 & 0.000 & 0.000 & 0.000 & 0.000 & 0.000 & 0.000 & 0.000 & 0.000 & 0.000 & 0.000 & 0.000 & 0.000 & 0.000 & 0.000 & 0.179 & 0.000 & 0.000 & 0.179 & 0.705 & 0.000 & 0.705 \\
\hline-0.96 & $216 \mathrm{~A}$ & 0 & 0.000 & 0.000 & 0.000 & 0.000 & 0.000 & 0.000 & 0.000 & 0.000 & 0.000 & 0.000 & 0.000 & 0.000 & 0.000 & 0.000 & 0.678 & 0.018 & 0.000 & 0.659 & 0.146 & 0.000 & 0.146 \\
\hline-1.00 & $215 \mathrm{~A}$ & 0 & 0.000 & 0.000 & 0.000 & 0.000 & 0.000 & 0.000 & 0.000 & 0.000 & 0.000 & 0.000 & 0.000 & 0.000 & 0.000 & 0.000 & 0.820 & 0.033 & 0.000 & 0.788 & 0.000 & 0.000 & 0.000 \\
\hline-1.13 & $214 \mathrm{~A}$ & 0 & 0.106 & 0.078 & 0.010 & 0.018 & 0.000 & 0.000 & 0.000 & 0.000 & 0.000 & 0.000 & 0.017 & 0.000 & 0.017 & 0.000 & 0.681 & 0.036 & 0.000 & 0.645 & 0.000 & 0.000 & 0.000 \\
\hline-1.37 & $213 \mathrm{~A}$ & 0 & 0.472 & 0.395 & 0.077 & 0.000 & 0.076 & 0.000 & 0.076 & 0.081 & 0.000 & 0.081 & 0.079 & 0.030 & 0.050 & 0.000 & 0.220 & 0.071 & 0.034 & 0.115 & 0.000 & 0.000 & 0.000 \\
\hline-1.60 & $212 \mathrm{~A}$ & 0 & 0.471 & 0.337 & 0.000 & 0.134 & 0.049 & 0.000 & 0.049 & 0.044 & 0.000 & 0.044 & 0.039 & 0.000 & 0.029 & 0.010 & 0.055 & 0.000 & 0.000 & 0.055 & 0.167 & 0.167 & 0.000 \\
\hline-2.52 & $211 \mathrm{~A}$ & 0 & 0.217 & 0.217 & 0.000 & 0.000 & 0.000 & 0.000 & 0.000 & 0.000 & 0.000 & 0.000 & 0.014 & 0.000 & 0.014 & 0.000 & 0.000 & 0.000 & 0.000 & 0.000 & 0.645 & 0.287 & 0.358 \\
\hline-3.83 & $210 \mathrm{~A}$ & 2 & 0.734 & 0.734 & 0.000 & 0.000 & 0.106 & 0.000 & 0.106 & 0.070 & 0.000 & 0.070 & 0.000 & 0.000 & 0.000 & 0.000 & 0.000 & 0.000 & 0.000 & 0.000 & 0.045 & 0.045 & 0.000 \\
\hline-4.04 & $209 \mathrm{~A}$ & 2 & 0.642 & 0.642 & 0.000 & 0.000 & 0.128 & 0.000 & 0.128 & 0.135 & 0.000 & 0.135 & 0.000 & 0.000 & 0.000 & 0.000 & 0.040 & 0.040 & 0.000 & 0.000 & 0.000 & 0.000 & 0.000 \\
\hline-5.11 & $208 \mathrm{~A}$ & 2 & 0.130 & 0.130 & 0.000 & 0.000 & 0.055 & 0.000 & 0.055 & 0.179 & 0.000 & 0.179 & 0.165 & 0.019 & 0.117 & 0.029 & 0.082 & 0.082 & 0.000 & 0.000 & 0.204 & 0.062 & 0.141 \\
\hline-5.36 & $207 \mathrm{~A}$ & 2 & 0.000 & 0.000 & 0.000 & 0.000 & 0.000 & 0.000 & 0.000 & 0.000 & 0.000 & 0.000 & 0.000 & 0.000 & 0.000 & 0.000 & 0.828 & 0.693 & 0.135 & 0.000 & 0.000 & 0.000 & 0.000 \\
\hline-5.47 & $206 \mathrm{~A}$ & 2 & 0.242 & 0.242 & 0.000 & 0.000 & 0.200 & 0.000 & 0.200 & 0.178 & 0.000 & 0.178 & 0.045 & 0.000 & 0.024 & 0.021 & 0.000 & 0.000 & 0.000 & 0.000 & 0.147 & 0.017 & 0.130 \\
\hline-5.57 & $205 \mathrm{~A}$ & 2 & 0.014 & 0.014 & 0.000 & 0.000 & 0.011 & 0.000 & 0.011 & 0.291 & 0.000 & 0.291 & 0.000 & 0.000 & 0.000 & 0.000 & 0.510 & 0.000 & 0.000 & 0.510 & 0.000 & 0.000 & 0.000 \\
\hline-5.64 & $204 \mathrm{~A}$ & 2 & 0.000 & 0.000 & 0.000 & 0.000 & 0.000 & 0.000 & 0.000 & 0.000 & 0.000 & 0.000 & 0.000 & 0.000 & 0.000 & 0.000 & 0.795 & 0.000 & 0.000 & 0.795 & 0.041 & 0.000 & 0.041 \\
\hline-5.69 & $203 \mathrm{~A}$ & 2 & 0.033 & 0.033 & 0.000 & 0.000 & 0.000 & 0.000 & 0.000 & 0.043 & 0.000 & 0.043 & 0.000 & 0.000 & 0.000 & 0.000 & 0.680 & 0.000 & 0.000 & 0.680 & 0.024 & 0.000 & 0.024 \\
\hline-5.72 & $202 \mathrm{~A}$ & 2 & 0.012 & 0.000 & 0.000 & 0.012 & 0.111 & 0.000 & 0.111 & 0.178 & 0.000 & 0.178 & 0.000 & 0.000 & 0.000 & 0.000 & 0.443 & 0.000 & 0.000 & 0.443 & 0.000 & 0.000 & 0.000 \\
\hline-5.82 & $201 \mathrm{~A}$ & 2 & 0.000 & 0.000 & 0.000 & 0.000 & 0.148 & 0.000 & 0.148 & 0.155 & 0.000 & 0.155 & 0.000 & 0.000 & 0.000 & 0.000 & 0.506 & 0.000 & 0.000 & 0.506 & 0.000 & 0.000 & 0.000 \\
\hline-6.00 & $200 \mathrm{~A}$ & 2 & 0.058 & 0.058 & 0.000 & 0.000 & 0.488 & 0.000 & 0.488 & 0.132 & 0.000 & 0.132 & 0.000 & 0.000 & 0.000 & 0.000 & 0.116 & 0.000 & 0.000 & 0.116 & 0.000 & 0.000 & 0.000 \\
\hline-6.04 & $199 \mathrm{~A}$ & 2 & 0.047 & 0.047 & 0.000 & 0.000 & 0.063 & 0.000 & 0.063 & 0.010 & 0.000 & 0.010 & 0.057 & 0.000 & 0.000 & 0.057 & 0.037 & 0.000 & 0.000 & 0.037 & 0.475 & 0.000 & 0.475 \\
\hline-6.11 & $198 \mathrm{~A}$ & 2 & 0.028 & 0.028 & 0.000 & 0.000 & 0.043 & 0.000 & 0.043 & 0.027 & 0.000 & 0.027 & 0.287 & 0.000 & 0.028 & 0.259 & 0.000 & 0.000 & 0.000 & 0.000 & 0.304 & 0.000 & 0.304 \\
\hline
\end{tabular}




\begin{tabular}{|c|c|c|c|c|c|c|c|c|c|c|c|c|c|c|c|c|c|c|c|c|c|c|c|}
\hline-6.19 & $197 \mathrm{~A}$ & 2 & 0.138 & 0.116 & 0.000 & 0.022 & 0.048 & 0.000 & 0.048 & 0.383 & 0.000 & 0.383 & 0.122 & 0.000 & 0.060 & 0.062 & 0.034 & 0.022 & 0.000 & 0.012 & 0.000 & 0.000 & 0.000 \\
\hline-6.22 & $196 \mathrm{~A}$ & 2 & 0.043 & 0.043 & 0.000 & 0.000 & 0.282 & 0.000 & 0.282 & 0.032 & 0.000 & 0.032 & 0.261 & 0.000 & 0.058 & 0.203 & 0.000 & 0.000 & 0.000 & 0.000 & 0.046 & 0.000 & 0.046 \\
\hline-6.32 & $195 \mathrm{~A}$ & 2 & 0.025 & 0.025 & 0.000 & 0.000 & 0.015 & 0.000 & 0.015 & 0.000 & 0.000 & 0.000 & 0.568 & 0.000 & 0.131 & 0.437 & 0.000 & 0.000 & 0.000 & 0.000 & 0.000 & 0.000 & 0.000 \\
\hline-6.47 & $194 \mathrm{~A}$ & 2 & 0.090 & 0.061 & 0.000 & 0.029 & 0.318 & 0.000 & 0.318 & 0.371 & 0.000 & 0.371 & 0.000 & 0.000 & 0.000 & 0.000 & 0.000 & 0.000 & 0.000 & 0.000 & 0.000 & 0.000 & 0.000 \\
\hline-6.69 & $193 \mathrm{~A}$ & 2 & 0.000 & 0.000 & 0.000 & 0.000 & 0.000 & 0.000 & 0.000 & 0.000 & 0.000 & 0.000 & 0.738 & 0.000 & 0.000 & 0.738 & 0.000 & 0.000 & 0.000 & 0.000 & 0.000 & 0.000 & 0.000 \\
\hline-6.74 & $192 \mathrm{~A}$ & 2 & 0.000 & 0.000 & 0.000 & 0.000 & 0.000 & 0.000 & 0.000 & 0.016 & 0.000 & 0.016 & 0.738 & 0.000 & 0.000 & 0.738 & 0.000 & 0.000 & 0.000 & 0.000 & 0.000 & 0.000 & 0.000 \\
\hline-6.80 & $191 \mathrm{~A}$ & 2 & 0.010 & 0.010 & 0.000 & 0.000 & 0.013 & 0.000 & 0.013 & 0.000 & 0.000 & 0.000 & 0.598 & 0.000 & 0.010 & 0.587 & 0.000 & 0.000 & 0.000 & 0.000 & 0.045 & 0.000 & 0.045 \\
\hline-6.84 & $190 \mathrm{~A}$ & 2 & 0.012 & 0.012 & 0.000 & 0.000 & 0.010 & 0.000 & 0.010 & 0.000 & 0.000 & 0.000 & 0.481 & 0.000 & 0.018 & 0.463 & 0.013 & 0.013 & 0.000 & 0.000 & 0.135 & 0.060 & 0.075 \\
\hline-6.91 & $189 \mathrm{~A}$ & 2 & 0.000 & 0.000 & 0.000 & 0.000 & 0.000 & 0.000 & 0.000 & 0.000 & 0.000 & 0.000 & 0.689 & 0.000 & 0.015 & 0.673 & 0.000 & 0.000 & 0.000 & 0.000 & 0.000 & 0.000 & 0.000 \\
\hline-6.98 & $188 \mathrm{~A}$ & 2 & 0.000 & 0.000 & 0.000 & 0.000 & 0.021 & 0.000 & 0.021 & 0.000 & 0.000 & 0.000 & 0.524 & 0.000 & 0.023 & 0.500 & 0.000 & 0.000 & 0.000 & 0.000 & 0.125 & 0.040 & 0.085 \\
\hline-7.06 & $187 \mathrm{~A}$ & 2 & 0.066 & 0.066 & 0.000 & 0.000 & 0.000 & 0.000 & 0.000 & 0.000 & 0.000 & 0.000 & 0.321 & 0.000 & 0.018 & 0.303 & 0.000 & 0.000 & 0.000 & 0.000 & 0.303 & 0.107 & 0.195 \\
\hline-7.33 & $186 \mathrm{~A}$ & 2 & 0.116 & 0.103 & 0.013 & 0.000 & 0.216 & 0.000 & 0.216 & 0.129 & 0.000 & 0.129 & 0.000 & 0.000 & 0.000 & 0.000 & 0.000 & 0.000 & 0.000 & 0.000 & 0.312 & 0.222 & 0.090 \\
\hline-7.41 & $185 \mathrm{~A}$ & 2 & 0.019 & 0.019 & 0.000 & 0.000 & 0.000 & 0.000 & 0.000 & 0.000 & 0.000 & 0.000 & 0.608 & 0.000 & 0.000 & 0.608 & 0.035 & 0.035 & 0.000 & 0.000 & 0.000 & 0.000 & 0.000 \\
\hline-7.55 & $184 \mathrm{~A}$ & 2 & 0.291 & 0.291 & 0.000 & 0.000 & 0.158 & 0.000 & 0.158 & 0.130 & 0.000 & 0.130 & 0.000 & 0.000 & 0.000 & 0.000 & 0.013 & 0.013 & 0.000 & 0.000 & 0.184 & 0.082 & 0.102 \\
\hline-7.81 & $183 \mathrm{~A}$ & 2 & 0.000 & 0.000 & 0.000 & 0.000 & 0.000 & 0.000 & 0.000 & 0.000 & 0.000 & 0.000 & 0.526 & 0.000 & 0.000 & 0.526 & 0.087 & 0.000 & 0.000 & 0.087 & 0.000 & 0.000 & 0.000 \\
\hline-7.85 & $182 \mathrm{~A}$ & 2 & 0.000 & 0.000 & 0.000 & 0.000 & 0.000 & 0.000 & 0.000 & 0.000 & 0.000 & 0.000 & 0.000 & 0.000 & 0.000 & 0.000 & 0.763 & 0.135 & 0.000 & 0.628 & 0.000 & 0.000 & 0.000 \\
\hline-7.89 & $181 \mathrm{~A}$ & 2 & 0.042 & 0.042 & 0.000 & 0.000 & 0.015 & 0.000 & 0.015 & 0.000 & 0.000 & 0.000 & 0.000 & 0.000 & 0.000 & 0.000 & 0.552 & 0.028 & 0.000 & 0.524 & 0.012 & 0.000 & 0.012 \\
\hline-7.92 & $180 \mathrm{~A}$ & 2 & 0.000 & 0.000 & 0.000 & 0.000 & 0.000 & 0.000 & 0.000 & 0.000 & 0.000 & 0.000 & 0.238 & 0.000 & 0.000 & 0.238 & 0.297 & 0.000 & 0.000 & 0.297 & 0.000 & 0.000 & 0.000 \\
\hline-7.98 & $179 \mathrm{~A}$ & 2 & 0.000 & 0.000 & 0.000 & 0.000 & 0.000 & 0.000 & 0.000 & 0.000 & 0.000 & 0.000 & 0.681 & 0.000 & 0.000 & 0.681 & 0.000 & 0.000 & 0.000 & 0.000 & 0.000 & 0.000 & 0.000 \\
\hline-8.02 & $178 \mathrm{~A}$ & 2 & 0.000 & 0.000 & 0.000 & 0.000 & 0.000 & 0.000 & 0.000 & 0.000 & 0.000 & 0.000 & 0.533 & 0.000 & 0.000 & 0.533 & 0.026 & 0.014 & 0.000 & 0.012 & 0.000 & 0.000 & 0.000 \\
\hline-8.05 & $177 \mathrm{~A}$ & 2 & 0.010 & 0.010 & 0.000 & 0.000 & 0.000 & 0.000 & 0.000 & 0.000 & 0.000 & 0.000 & 0.000 & 0.000 & 0.000 & 0.000 & 0.502 & 0.015 & 0.000 & 0.488 & 0.000 & 0.000 & 0.000 \\
\hline-8.07 & $176 \mathrm{~A}$ & 2 & 0.014 & 0.014 & 0.000 & 0.000 & 0.000 & 0.000 & 0.000 & 0.000 & 0.000 & 0.000 & 0.023 & 0.000 & 0.000 & 0.023 & 0.370 & 0.022 & 0.000 & 0.348 & 0.000 & 0.000 & 0.000 \\
\hline
\end{tabular}


S23: References

(1) Sanford, M. S.; Love, J. A.; Grubbs, R. H. Organomet. 2001, 20, 53145318.

(2) Love, J. A.; Morgan, J. P.; Trnka, T. M.; Grubbs, R. H. Angew. Chem. Int. Ed. 2002, 41, 4035-4037.

(3) Trnka, T. M.; Dias, E. L.; Day, M. W.; Grubbs, R. H. Arkivoc 2002, 13, 2841.

(4) Carlson, R. G.; Gile, M. A.; Heppert, J. A.; Mason, M. H.; Powell, D. R.; Velde, D. V.; Vilain, J. M. J. Am. Chem. Soc. 2002, 124, 1580-1581.

(5) Romero, P. E.; Piers, W. E.; McDonald, R. Angew. Chem. Int. Ed. 2004, 43, 6161-6165.

(6) Webb, S. M. Physica Scripta 2005, T115, 1011-1014.

(7) PeakFit, 4.12; SeaSolve Software Inc. 2003.

(8) George, G. N., EXAFSPAK http://ssrl.slac.stanford.edu/EXAFSPAK.html. 2001.

(9) Delgado-Jaime, M. U.; Conrad, J. C.; Fogg, D. E.; Kennepohl, P. Inorg. Chim. Acta 2006, 359, 3042-3047.

(10) Becke, A. D. Phys. Rev. A 1988, 38, 3098.

(11) Perdew, J. P. Phys. Rev. B 1986, 34, 7406.

(12) Perdew, J. P. Phys. Rev. B 1986, 33, 8822.

(13) Snijders, J. G.; Baerdens, E. J. A. Data Nucl. Data Tables 1982, 26, 483509.

(14) Baerdens, E. J.; Ellis, D. E.; Ros, P. Chem. Phys. 1973, 2, 41-51.

(15) ADF2005.01 Theoretical Chemistry, Vrije Universiteit: Amsterdam, The Netherlands.

(16) Guerra, C. F.; Snijders, J. G.; te Velde, G.; Baerdens, E. J. Theor. Chem. Acc. 1998, 99, 391-403.

(17) te Velde, G.; Bickelhaupt, F. M.; Baerends, E. J.; Fonseca Guerra, C.; van Gisbergen, S. J. A.; Snijders, J. G.; Ziegler, T. J. Comp. Chem. 2001, 22, 931-967. 